\title{
The standard upwind compact difference schemes for incompressible flow simulations
}

\author{
Ping Fan ${ }^{\mathrm{a}, \mathrm{b}}$ \\ a Institute of Process Engineering, Chinese Academy of Sciences, Beijing 100190, China \\ ${ }^{\mathrm{b}}$ Institute of Mechanics, Chinese Academy of Sciences, Beijing 100190, China
}

\section{A R T I C L E I N F O}

\section{Article history:}

Received 20 November 2015

Received in revised form 5 May 2016

Accepted 16 June 2016

Available online 22 June 2016

\section{Keywords:}

Compact difference scheme

Upwind scheme

Upwind compact scheme

Incompressible flows

Lid-driven cavity

\begin{abstract}
A B S T R A C T
Compact difference schemes have been used extensively for solving the incompressible Navier-Stokes equations. However, the earlier formulations of the schemes are of central type (called central compact schemes, CCS), which are dispersive and susceptible to numerical instability. To enhance stability of CCS, the optimal upwind compact schemes (OUCS) are developed recently by adding high order dissipative terms to CCS. In this paper, it is found that OUCS are essentially not of the upwind type because they do not use upwind-biased but central type of stencils. Furthermore, OUCS are not the most optimal since orders of accuracy of OUCS are at least one order lower than the maximum achievable orders. New upwind compact schemes (called standard upwind compact schemes, SUCS) are developed in this paper. In contrast to OUCS, SUCS are constructed based completely on upwind-biased stencils and hence can gain adequate numerical dissipation with no need for introducing optimization calculations. Furthermore, SUCS can achieve the maximum achievable orders of accuracy and hence be more compact than OUCS. More importantly, SUCS have prominent advantages on combining the stable and high resolution properties which are demonstrated from the global spectral analyses and typical numerical experiments.
\end{abstract}

(C) 2016 Elsevier Inc. All rights reserved.

\section{Introduction}

Compact difference schemes have been widely used to compute problems involving compressible and incompressible flows and several other practical applications [1-5]. Compared to the explicit type schemes of the same order of accuracy, high order compact difference schemes have the advantage of not only avoiding cumbersomely large size stencils but also providing spectral-like resolution characteristics. Compact schemes are usually evaluated separately for first and second derivative as in [6]. In another variant of compact scheme referred to as the combined compact difference (CCD) scheme, the derivatives are obtained simultaneously using Hermitian polynomials [7-11].

The earlier formulations of the compact schemes in [6] are essentially of the central type, which are known to be dispersive and susceptible to numerical instability in strongly convective flows. Of late, upwind compact schemes (UCS) have received attention as they are immune to numerical instability induced by the spurious waves and hence can enhance the convective stability. The existing UCS schemes for solving incompressible flow problems are usually referred to as the optimal upwind compact schemes (OUCS) [12-15]. The OUCS are constructed by adding high order dissipative terms to CCS

E-mail addresses: pfan@ipe.ac.cn, fanping@imech.ac.cn. 
Table 1

Coefficients of CCS scheme (tri-diagonal) by Lele [6].

\begin{tabular}{llllllllll}
\hline Order & $\alpha_{-1}$ & $\alpha_{1}$ & $c_{-3}$ & $c_{-2}$ & $c_{-1}$ & $c_{1}$ & $c_{2}$ & $c_{3}$ & TE \\
\hline 4 & $\frac{1}{4}$ & $\frac{1}{4}$ & & & $\frac{-3}{4}$ & $\frac{3}{4}$ & & & $\frac{-h^{4}}{5 !}$ \\
6 & $\frac{1}{3}$ & $\frac{1}{3}$ & & $\frac{-1}{36}$ & $\frac{-7}{9}$ & $\frac{7}{9}$ & $\frac{1}{36}$ & & $\frac{4 h^{6}}{7 !}$ \\
8 & $\frac{3}{8}$ & $\frac{3}{8}$ & $\frac{-1}{480}$ & $\frac{-1}{20}$ & $\frac{-25}{32}$ & $\frac{25}{32}$ & $\frac{1}{20}$ & $\frac{1}{480}$ & $\frac{12 h^{7}}{8 !}$ \\
\hline
\end{tabular}

and carrying out optimization calculations through using a free parameter after a less than maximum order of accuracy is accepted.

Actually, the OUCS schemes should be classified into the central type not the upwind type since they do not use upwindbiased but central type of stencils. Furthermore, coefficients of these schemes have to be determined from optimization calculations and sometimes are not analytic [14,15]. If not written with a sufficient accuracy comparable to that of the schemes the non-analytic coefficients can produce new additional numerical errors and decrease the overall accuracy of the schemes. More importantly, these optimized schemes are not the most optimal since orders of accuracy of these schemes are at least one order lower than the maximum achievable orders for the stencils they used $[12,14,15]$.

In this work, a series of new high resolution UCS schemes are developed with orders of accuracy ranging from third to eleventh. In contrast to the existing OUCS schemes, the new schemes are constructed based completely on upwind-biased stencils as is done in the classic explicit upwind schemes. As a consequence, the new schemes are termed as the standard upwind compact schemes (SUCS) to distinguish with the OUCS schemes. In SUCS numerical dissipation can be gained automatically due to the dissipative nature of the schemes and numerical stability can be guaranteed sufficiently with no need for introducing additional free parameters to run optimization calculations. Moreover, the SUCS schemes can make the best use of the stencils to achieve the maximum attainable orders which makes the SUCS schemes more compact than the OUCS schemes. The SUCS have prominent advantages on combining the stable and high resolution properties which are demonstrated from the global spectral analyses and typical numerical experiments.

In addition to the explicit presentations of SUCS on uniform grids, this paper also derives the generalized formulations for the new schemes. It should be mentioned that derivations for the generalized formulations of the compact schemes are oft attempted by using the Hermite interpolation polynomial based methods [8,9,17]. However, this kind of methodology is not quite perfect due to the following reasons. First, the derived coefficients of the schemes are not fully explicit since derivative operators are still contained in the final expressions [17]. Second, also the most important, from this method the truncation errors (TE) cannot be obtained. As known to all, the TE plays a key role in elucidating numerical characteristics of a difference scheme because the basic numerical features including order of accuracy and dissipation or dispersion features can be learned directly from the TE. Without the explicit expressions of TE, any discussion on the accuracy of a difference scheme is meaningless. To overcome these limitations of the Hermite polynomial method, this paper uses a stepwise weighted summation method and applies the mathematical induction principle to derive the generalized formulations for SUCS. With this method, the TE can be obtained concurrently with the fully explicit presentations of the coefficients of the schemes. This facilitates a direct grasp of the numerical features of SUCS schemes and provides a sound theoretical foundation for its applications.

The paper is organized as follows. Section 2 presents coefficients of the SUCS schemes on uniform grids and examines connections between SUCS and OUCS schemes. Section 3 conducts the global spectral analysis for the SUCS schemes. In section 4 numerical experiments are carried out to demonstrate capabilities of SUCS on combining the stable and high resolution properties. The generalized formulations of SUCS schemes are derived in section 5. Section 6 concludes the paper.

\section{The standard upwind compact schemes on uniform grids}

For a constant grid spacing $h$, compact scheme for first derivative of $u$ at $x_{0}$ can be written in a generic form as

$$
u_{0}^{\prime}+\sum_{i \in \Omega_{1}} \alpha_{i} u_{i}^{\prime}=\frac{1}{h} \sum_{j \in \Omega_{2}} c_{j} u_{j}
$$

where $\Omega_{1}$ and $\Omega_{2}$ denote the point sets on which the derivatives and function values are defined, respectively. If using the following symmetric assumptions $\alpha_{-i}=\alpha_{i}, c_{-j}=c_{j}$ and $c_{0}=0$, the classic central compact schemes (CCS) can be derived. Through Taylor series expanding (1) and matching coefficients of various orders, the CCS schemes of different orders have been constructed by Lele [6]. Table 1 lists parts of the results from [6] with the purpose of aiding in the latter study for the connections between the OUCS and SUCS schemes

In order to enhance numerical dissipations of the CCS schemes, the OUCS schemes are developed by adding high order dissipative terms to the CCS schemes. Table 2 lists coefficients of the OUCS schemes derived by Zhong [12], referred to as OUCS-Z, in which $\lambda$ is a free parameter chosen to stabilize the scheme. For the 3rd-order, 5th-order and 7th-order OUCS-Z schemes, the optimized values of $\lambda$ are found to be $1 / 4,-1$, and 36 , respectively [12]. Together with the latter variants 
Table 2

Coefficients of OUCS-Z scheme by Zhong [12].

\begin{tabular}{|c|c|c|c|c|c|}
\hline Order & $\alpha_{ \pm 1}$ & $c_{ \pm 3}$ & $c_{ \pm 2}$ & $c_{ \pm 1}$ & $c_{0}$ \\
\hline 3 & $\frac{1}{4} \mp \frac{\lambda}{4}$ & & & $\pm \frac{3}{4}-\frac{\lambda}{2}$ & $\lambda$ \\
\hline 5 & $\frac{1}{3} \pm \frac{\lambda}{12}$ & & $\pm \frac{1}{36}+\frac{\lambda}{72}$ & $\pm \frac{7}{9}+\frac{\lambda}{9}$ & $-\frac{\lambda}{4}$ \\
\hline 7 & $\frac{3}{8} \mp \frac{\lambda}{96}$ & $\mp \frac{1}{480}+\frac{\lambda}{5760}$ & $\pm \frac{1}{20}-\frac{\lambda}{360}$ & $\pm \frac{25}{32}-\frac{13 \lambda}{1152}$ & $\frac{\lambda}{36}$ \\
\hline
\end{tabular}

Table 3

Coefficients of OUCS-S ${ }^{\mathrm{a}}$ scheme by Sengupta et al. [13], OUCS-B schemes by Bhumkar et al. [15] and OUCS-D by De and Eswaran [14].

\begin{tabular}{llll}
\hline & 5th-order OUCS-S & 5th-order OUCS-B & 5th-order OUCS-D \\
\hline$\alpha_{-2}$ & & & 0.0895 \\
$\alpha_{-1}$ & $23 / 54$ & 0.5406319255184851 & 0.57967 \\
$\alpha_{1}$ & $13 / 54$ & 0.1260347411481814 & 0.57967 \\
$\alpha_{2}$ & & & 0.0895 \\
$c_{-3}$ & & & $-0.00559+0.0014 \lambda$ \\
$c_{-2}$ & $-7 / 162$ & -0.0623275431419697 & $-0.25154-0.0083 \lambda$ \\
$c_{-1}$ & $-46 / 81$ & -1.0541759006913135 & $-0.6494+0.0208 \lambda$ \\
$c_{0}$ & $5 / 18$ & 0.6218957765554555 & $-0.0278 \lambda$ \\
$c_{1}$ & $26 / 81$ & 0.5013796548642419 & $0.6494+0.0208 \lambda$ \\
$c_{2}$ & $1 / 81$ & -0.0067719875864141 & $0.25154-0.0083 \lambda$ \\
$c_{3}$ & & & $0.00559+0.0014 \lambda$ \\
\hline
\end{tabular}

a The OUCS-S scheme corresponds to the OUCS2 scheme in [13].

Table 4a

Coefficients of left-biased tri-diagonal SUCS schemes.

\begin{tabular}{|c|c|c|c|c|c|c|c|c|c|c|c|}
\hline Order & $\alpha_{-1}$ & $\alpha_{1}$ & $c_{-4}$ & $c_{-3}$ & $c_{-2}$ & $c_{-1}$ & $c_{0}$ & $c_{1}$ & $c_{2}$ & $c_{3}$ & $\mathrm{TE}$ \\
\hline 3 & $\frac{1}{2}$ & & & & & $\frac{-5}{4}$ & 1 & $\frac{1}{4}$ & & & $\frac{h^{3}}{4 !}$ \\
\hline 5 & $\frac{1}{2}$ & $\frac{1}{6}$ & & & $\frac{-1}{18}$ & -1 & $\frac{1}{2}$ & $\frac{5}{9}$ & & & $\frac{-2 h^{5}}{6 !}$ \\
\hline 7 & $\frac{1}{2}$ & $\frac{1}{4}$ & & $\frac{1}{240}$ & $\frac{-1}{12}$ & $\frac{-11}{12}$ & $\frac{1}{3}$ & $\frac{31}{48}$ & $\frac{1}{60}$ & & $\frac{12 h^{7}}{8 !}$ \\
\hline 9 & $\frac{1}{2}$ & $\frac{3}{10}$ & $\frac{-1}{2100}$ & $\frac{1}{120}$ & $\frac{-1}{10}$ & $\frac{-7}{8}$ & $\frac{1}{4}$ & $\frac{959}{1400}$ & $\frac{1}{30}$ & $\frac{-1}{840}$ & $\frac{-144 h^{9}}{10 !}$ \\
\hline
\end{tabular}

Table 4b

Coefficients of right-biased tri-diagonal SUCS schemes.

\begin{tabular}{lcl}
\hline Order & $\alpha_{i}$ & $c_{j}$ \\
\hline $2 r+1$ & $\alpha_{i}=\alpha_{-i}$ & $c_{j}=-c_{-j}$ \\
$(1 \leq r \leq 4)$ & $(i \in\{-1,1\})$ & $(0 \leq j \leq r)$ \\
$\alpha_{-i}$ and $c_{-j}$ correspond to those listed in Table 4a
\end{tabular}

developed by Sengupta et al. [13], referred to as OUCS-S schemes, the orders of accuracy of these OUCS schemes are all one order lower than their maximum achievable orders.

In Table 3 coefficients of another 5th-order OUCS scheme derived by De and Eswaran [14], referred to as OUCS-D, are presented. It is easy to identify that the maximum achievable order of OUCS-D scheme is 10, much higher than the real order of the scheme. Table 3 also presents parts of the results derived by Bhumkar et al. [15]. In this latest variant of OUCS scheme, referred to as OUCS-B, the coefficients are determined via minimizing an error function and the optimized values of these coefficients are not analytic. New numerical errors can be produced due to the non-analytic property of the coefficients which may further decrease the overall accuracy of the scheme. It can also be seen that the maximum achievable order of the 5th-order OUCS-B scheme is one order higher than the real order of the scheme.

As a common feature of the above-mentioned OUCS schemes, these schemes are constructed exclusively based on a central stencil while the maximum achievable orders of accuracy are not achieved. To overcome this prominent weakness of OUCS schemes, a new upwind compact scheme for incompressible flows is developed in this paper. This new scheme is termed as the standard upwind compact scheme (SUCS), considering that the scheme selects an upwind-biased stencil as have been used in the classic explicit upwind schemes. The derivation of the schemes is detailed in section 5.

Tables $4 \mathrm{a}$ and $4 \mathrm{~b}$ present, respectively, coefficients of the left-biased and right-biased tri-diagonal SUCS schemes with accuracy ranging from the 3rd-order to the 9th-order. In Tables $5 \mathrm{a}$ and $5 \mathrm{~b}$ coefficients of the left-biased and right-biased penta-diagonal SUCS schemes with orders of accuracy ranging from 7th to 11th are given, respectively. According to the standard definition of the upwind scheme, the left-biased scheme should be used when the convection velocity is positive, while a right-biased version should be used when the convection velocity is negative. From comparing Table 4a with Table 3, it is clear that to obtain a same order of accuracy the SUCS scheme uses a smaller stencil than the OUCS scheme. Also, no 
Table 5a

Coefficients of left-biased penta-diagonal SUCS schemes.

\begin{tabular}{|c|c|c|c|c|c|c|c|c|c|c|c|c|c|}
\hline Order & $\alpha_{-2}$ & $\alpha_{-1}$ & $\alpha_{1}$ & $\alpha_{2}$ & $c_{-4}$ & $c_{-3}$ & $c_{-2}$ & $c_{-1}$ & $c_{0}$ & $c_{1}$ & $c_{2}$ & $c_{3}$ & $\mathrm{TE}$ \\
\hline 7 & $\frac{1}{18}$ & $\frac{2}{3}$ & $\frac{2}{9}$ & & & & $\frac{-47}{216}$ & $\frac{-8}{9}$ & $\frac{1}{2}$ & $\frac{16}{27}$ & $\frac{1}{72}$ & & $\frac{h^{7}}{7 !}$ \\
\hline 9 & $\frac{1}{12}$ & $\frac{2}{3}$ & $\frac{1}{3}$ & $\frac{1}{60}$ & & $\frac{-1}{300}$ & $\frac{-19}{72}$ & $\frac{-7}{9}$ & $\frac{1}{3}$ & $\frac{23}{36}$ & $\frac{131}{1800}$ & & $\frac{-48 h^{9}}{10 !}$ \\
\hline 11 & $\frac{1}{10}$ & $\frac{2}{3}$ & $\frac{2}{5}$ & $\frac{1}{30}$ & $\frac{1}{6300}$ & $\frac{-1}{150}$ & $\frac{-301}{1050}$ & $\frac{-91}{126}$ & $\frac{1}{4}$ & $\frac{679}{1050}$ & $\frac{371}{3150}$ & $\frac{1}{1050}$ & $\frac{48 h^{11}}{11 !}$ \\
\hline
\end{tabular}

Table 5b

Coefficients of right-biased penta-diagonal SUCS schemes.

\begin{tabular}{lll}
\hline Order & $\alpha_{i}$ & $c_{j}$ \\
\hline $2 r+1$ & $\alpha_{i}=\alpha_{-i}$ & $c_{j}=-c_{-j}$ \\
$(3 \leq r \leq 5)$ & $(i \in\{-1,-2,1,2\})$ & $(0 \leq j \leq r-1)$ \\
$\alpha_{-i}$ and $c_{-j}$ correspond to those listed in Table 5a \\
\hline
\end{tabular}

Table 6a

Coefficients of boundary closure schemes for the left boundary.

\begin{tabular}{|c|c|c|c|c|c|c|c|c|}
\hline Order & $\alpha_{1}$ & $c_{0}$ & $c_{1}$ & $c_{2}$ & $c_{3}$ & $c_{4}$ & $c_{5}$ & $\mathrm{TE}$ \\
\hline 3 & 2 & $\frac{-5}{2}$ & 2 & $\frac{1}{2}$ & & & & $\frac{h^{3}}{4 !}$ \\
\hline 4 & 3 & $\frac{-17}{6}$ & $\frac{3}{2}$ & $\frac{3}{2}$ & $\frac{-1}{6}$ & & & $\frac{-h^{4}}{5 !}$ \\
\hline 5 & 4 & $\frac{-37}{12}$ & $\frac{2}{3}$ & 3 & $\frac{-2}{3}$ & $\frac{1}{12}$ & & $\frac{h^{5}}{6 !}$ \\
\hline 6 & 5 & $\frac{-197}{60}$ & $\frac{-5}{12}$ & 5 & $\frac{-5}{3}$ & $\frac{5}{12}$ & $\frac{-1}{20}$ & $\frac{-h^{6}}{7 !}$ \\
\hline$p$ & $p-1$ & \multicolumn{6}{|c|}{$\begin{array}{l}c_{1}=(p-1)\left(2-\sum_{l=1}^{p-2} \frac{1}{l}\right) \\
c_{j}=\frac{(-1)^{j}}{j(j-1)} \frac{1}{j !} \frac{(p-1) !}{(p-1-j) !}, \quad j \in[2, p-1] \\
c_{0}=-\sum_{j=1}^{p-1} c_{j}\end{array}$} & $\frac{(-1)^{p+1} h^{p}}{(p+1) !}$ \\
\hline
\end{tabular}

Table 6b

Coefficients of boundary closure schemes for the right boundary.

\begin{tabular}{lll}
\hline Order & $\alpha_{-1}$ & $c_{-j}$ \\
\hline$p$ & $p-1$ & $c_{-j}=-c_{j}, 0 \leq j \leq p-1, c_{j}$ correspond to those listed in Table 6a \\
\hline
\end{tabular}

free parameter is introduced in the SUCS schemes, manifesting the SUCS schemes more strict and accurate than the OUCS schemes. Coefficients of the boundary closure schemes with arbitrary order of accuracy are listed in Tables 6a and 6b.

To further investigate differences between SUCS and OUCS schemes, an interesting connection among SUCS, OUCS-Z and CCS schemes is worthy of a special discussion. By substituting the optimized value of $\lambda\left(\lambda_{o p t}=1 / 4\right)$ for the 3rd-order OUCS-Z scheme, one can easily derive from Table 2 that $u_{0}^{\prime}$ written in the OUCS-Z scheme can be expressed as

$$
\left.u_{0}^{\prime}\right|_{\text {OUCS-Z }} ^{(3)}=\frac{-7 u_{-1}+2 u_{0}+5 u_{1}}{8 h}-\frac{5 u_{-1}^{\prime}+3 u_{1}^{\prime}}{16}
$$

which can be rewritten as

$$
\begin{aligned}
\left.u_{0}^{\prime}\right|_{\text {OUCS- } Z} ^{(3)} & =\frac{1}{4}\left(\frac{-5 u_{-1}+4 u_{0}+u_{1}}{4 h}-\frac{1}{2} u_{-1}^{\prime}\right)+\frac{3}{4}\left(\frac{3}{4} \frac{u_{1}-u_{-1}}{h}-\frac{u_{-1}^{\prime}+u_{1}^{\prime}}{4}\right) \\
& =\left.\frac{1}{4} u_{0}^{\prime}\right|_{\text {SUCS }} ^{(3)}+\left.\frac{3}{4} u_{0}^{\prime}\right|_{\text {CCS }} ^{(4)}
\end{aligned}
$$

The above formulation implies that the OUCS-Z scheme is essentially a linear superposition of a SUCS scheme of the same order and a higher order CCS scheme.

Similarly, from substitution of the optimal value -1 for $\lambda$ one can identify that the 5 th-order OUCS- $Z$ scheme can be expressed as a sum of 5th-order SUCS and 6th-order CCS schemes, that is, 


$$
\begin{aligned}
\left.u_{0}^{\prime}\right|_{\text {OUCS-Z }} ^{(5)}= & \frac{1}{h}\left(-\frac{u_{-2}}{24}-\frac{8 u_{-1}}{9}+\frac{u_{0}}{4}+\frac{2 u_{1}}{3}+\frac{u_{2}}{72}\right)-\frac{5 u_{-1}^{\prime}+3 u_{1}^{\prime}}{12} \\
= & \frac{1}{2}\left(\frac{-u_{-2}-18 u_{-1}+9 u_{0}+10 u_{1}}{18 h}-\frac{3 u_{-1}^{\prime}+u_{1}^{\prime}}{6}\right) \\
& +\frac{1}{2}\left(\frac{7}{9} \frac{u_{1}-u_{-1}}{h}+\frac{u_{2}-u_{-2}}{36 h}-\frac{u_{-1}^{\prime}+u_{1}^{\prime}}{3}\right) \\
= & \left.\frac{1}{2} u_{0}^{\prime}\right|_{\text {SUCS }} ^{(5)}+\left.\frac{1}{2} u_{0}^{\prime}\right|_{C C S} ^{(6)}
\end{aligned}
$$

Also, the 7th-order OUCS-Z scheme can be expressed as a sum of 7th-order SUCS and 8th-order CCS schemes with substitution of the optimal value 36 for $\lambda$, that is,

$$
\begin{aligned}
\left.u_{0}^{\prime}\right|_{\text {OUCS }-Z} ^{(7)}= & \frac{1}{h}\left(\frac{u_{-3}}{120}-\frac{3 u_{-2}}{20}-\frac{19}{16} u_{-1}+u_{0}+\frac{3}{8} u_{1}-\frac{u_{2}}{20}+\frac{u_{3}}{240}\right)-\frac{3 u_{-1}^{\prime}}{4} \\
= & 3\left(\frac{u_{-3}-20 u_{-2}-220 u_{-1}+80 u_{0}+155 u_{1}+4 u_{2}}{240 h}-\frac{2 u_{-1}^{\prime}+u_{1}^{\prime}}{4}\right) \\
& -2\left(\frac{u_{-3}-24 u_{-2}-375 u_{-1}+375 u_{1}+24 u_{2}-u_{3}}{480 h}-3 \frac{u_{-1}^{\prime}+u_{1}^{\prime}}{8}\right) \\
= & \left.3 u_{0}^{\prime}\right|_{\text {SUCS }} ^{(7)}-\left.2 u_{0}^{\prime}\right|_{\text {CCS }} ^{(8)}
\end{aligned}
$$

It is clear that the truncation errors in the second terms on the right hand side (RHS) of equations (3)-(5) are one order higher than those in the first terms and hence can be neglected. This means accuracy of the OUCS-Z schemes are determined primarily by the first terms on RHS of the above three equations. It can be learned from Eqs. (3)-(5) that the 7th-order OUCS-Z (OUCS-Z7) has 3 times higher truncation error than the 7th-order SUCS (SUCS7) but truncation errors of OUCS-Z3 and OUCS-Z5 are 4 times and 2 times lower than those of SUCS3 and SUCS5, respectively.

In general, the OUCS-Z schemes attain a same order of accuracy as the SUCS schemes but use larger stencils than those used by SUCS. This means that the SUCS is more compact than OUCS-Z.

Furthermore, it deserves to be mentioned that accuracy of OUCS-Z can be further enhanced by an analogous combination algorithm without enlarging its stencil. A series of new possible OUCS schemes can be constructed from the following combinations,

$$
\left.u_{0}^{\prime}\right|_{\text {OUCS-new }} ^{(2 r)}=\left.\alpha u_{0}^{\prime}\right|_{\text {SUCS }} ^{(2 r-1)}+\left.(1-\alpha) u_{0}^{\prime}\right|_{C C S} ^{(2 r)} \quad(r \geq 2)
$$

Apparently, the above new possible OUCS schemes can attain a higher order of accuracy than the existing OUCS-Z schemes provided that value of the free parameter $\alpha$ is taken properly (such as $\alpha=h^{1 / 2}$ ). This presents another evidence for showing that the OUCS schemes are not the most optimal and can be further improved with using the SUCS schemes proposed in this paper.

\section{Global spectral analysis}

In this section, the global spectral analysis (GSA) method used earlier in [13] is adopted to show the high resolution and stable capabilities of SUCS schemes. The theoretical foundation for GSA is described shortly as follows. The physical variables in physical plane and spectral plane can be related by

$$
u\left(x_{l}\right)=\int_{-k_{m}}^{k_{m}} U(k) e^{i k x_{l}} d k
$$

where $k_{m}=\pi / h$ denotes the Nyquist limit of the wavenumber. The first derivative of $u$ evaluated by the numerical method can be expressed as

$$
u^{\prime}\left(x_{l}\right)=\int_{-k_{m}}^{k_{m}} i k_{e q} U(k) e^{i k x_{l}} d k
$$

where the complex quantity $k_{e q}$ is the equivalent wavenumber. The deviations of the real and imaginary parts of $k_{e q}$ from the exact scaled wavenumber $k$ are usually served as indicators for the numerical dispersion and dissipation, respectively.

In this paper, $k_{e q}$ is computed with the method detailed in $[13,16]$ and values of $k_{e q} / k$ are plotted against $k h$ to elucidate the dispersion and dissipation accuracy of the schemes. Here, value of the node number is chosen to be 100 . To compare 


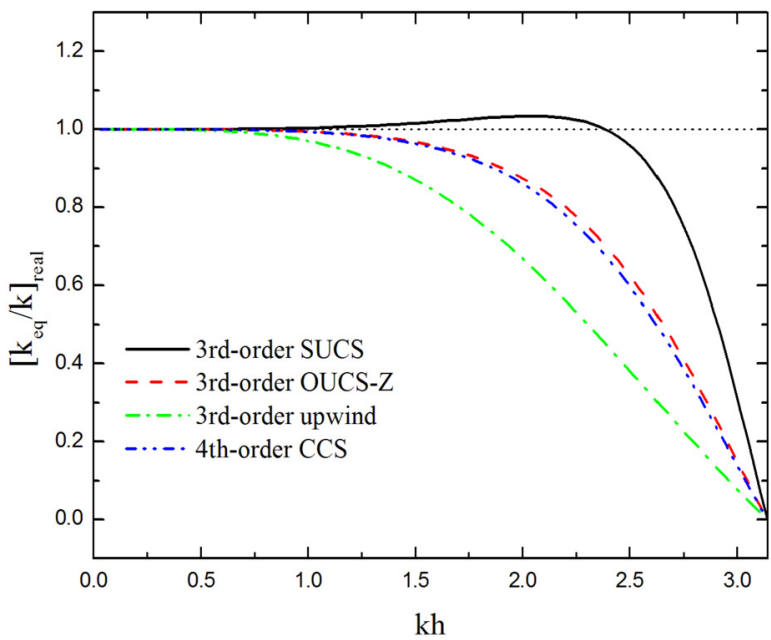

(a)

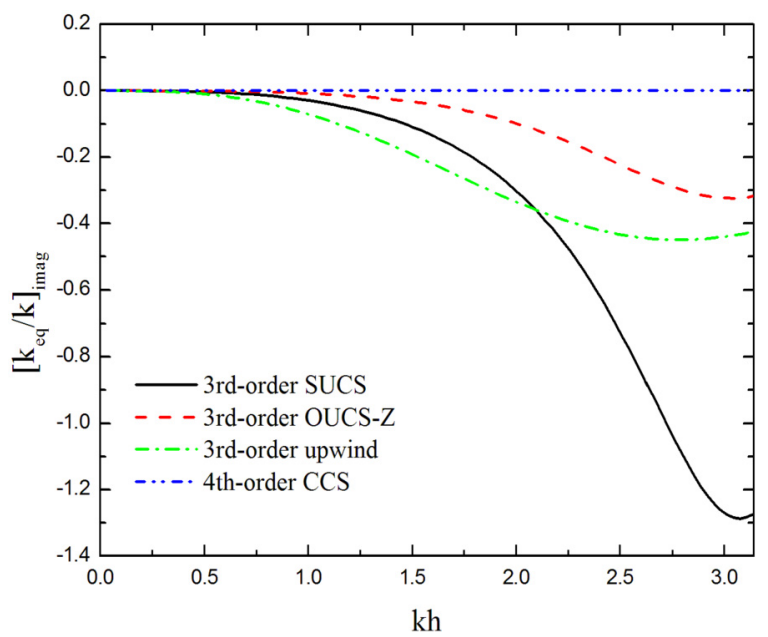

(b)

Fig. 1. (a) Comparison of the real part of $k_{e q} / k$ at the central node in the spectral plane computed by the 3rd-order SUCS, OUCS-Z, upwind and 4th-order CCS schemes (from Figs. 1 to 4 value of $h$ is taken as 0.01). (b) Comparison of the imaginary part of $k_{e q} / k$ at the central node in the spectral plane computed by the 3rd-order SUCS, OUCS-Z, upwind and 4th-order CCS schemes.

well with the existing OUCS schemes, the selected SUCS schemes are tri-diagonal. It is found that effects of the different boundary schemes are non-trivial only in limited regions close to the boundary. Therefore, in examining features of $k_{e q} / k$ at the central node the 4th-order explicit type boundary schemes are chosen typically. Before presenting results of GSA, it deserves to be noted that it is possible to do GSA for full domain analysis for each and every nodes. Here only the properties of interior stencil are shown for analyzed schemes. Also, it should be stressed that by spatial discretization, one can assess the trends of dissipation and dispersion, while a quantitative value can only be obtained by analyzing space-time discretization together.

In Figs. 1 the real and imaginary parts of $k_{e q} / k$ computed by the 3rd-order SUCS scheme at the central node in the spectral plane are plotted and compared with those using the 3rd-order OUCS-Z, 3rd-order upwind and 4th-order CCS schemes. As has been pointed out, the real part of $k_{e q} / k$ indicates the dispersion error of a scheme which is more accurate in spectral space provided the real part of $k_{e q} / k$ is more close to one. Seen from Fig. 1(a) where the real part of $k_{e q} / k$ is plotted, it is evident that the SUCS has the best dispersion accuracy among all of the schemes over the whole range of wavenumber. The upwind scheme presents the least dispersion accuracy, while the OUCS-Z is slightly better than CCS both of which occupy an intermediary position among the four schemes.

In Fig. 1(b), the imaginary part of $k_{e q} / k$ which depicts the numerical dissipation is plotted for different schemes. Evidently, the CCS has no dissipation errors while the dissipation accuracy of OUCS-Z is higher than those of the SUCS and upwind schemes over the whole range of wavenumber. At very high wavenumbers, the dissipation error of SUCS is the most significant which manifests the SUCS scheme has a strongest stability in damping out the small-scale spurious oscillations.

In Figs. 2 the real and imaginary parts of $k_{e q} / k$ computed by 5th-order SUCS scheme at the central node in the spectral plane are plotted and compared with those using 5th-order OUCS-Z, 5th-order OUCS-S, 5th-order upwind and 6th-order CCS schemes. The results exhibit a similar tendency as that shown in Figs. 1. The SUCS has the best dispersion accuracy over the whole range of wavenumber but presents the most significant dissipation errors for very high wavenumbers.

The results shown in these figures manifest a prominent property of SUCS schemes that the SUCS schemes not only have the highest resolution capability for the low and moderate wavenumbers but can also produce sufficient dissipations to attenuate the small-scale spurious oscillations at very high wavenumbers.

\section{Numerical experiments}

In order to test the newly developed SUCS schemes as well as demonstrate their capabilities on combining the stable and high resolution properties, the 1-D linear wave equation with discontinuous initial condition and the incompressible lid-driven cavity flows are simulated and the solutions are compared between the SUCS and other difference schemes.

\subsection{Solution of linear wave equation}

In this problem the first order linear wave equation

$$
\frac{\partial u}{\partial t}+c \frac{\partial u}{\partial x}=0
$$




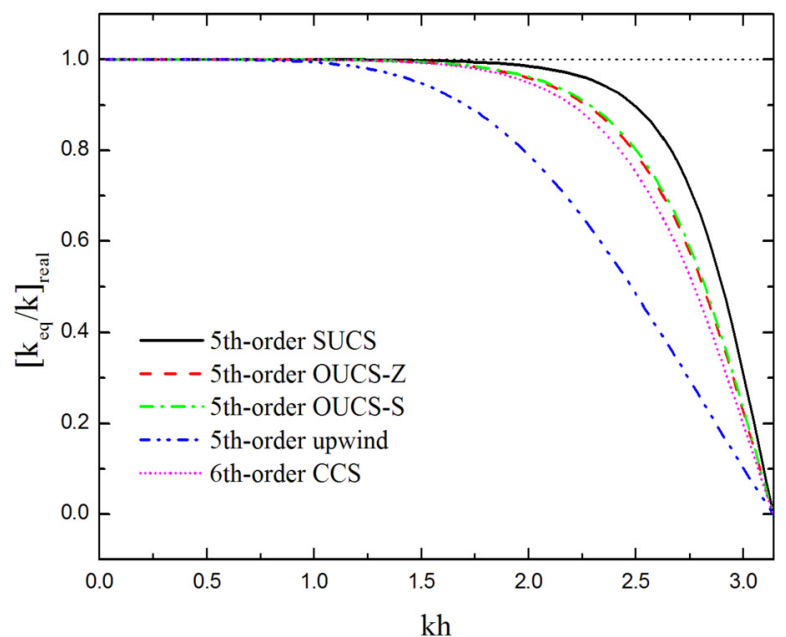

(a)

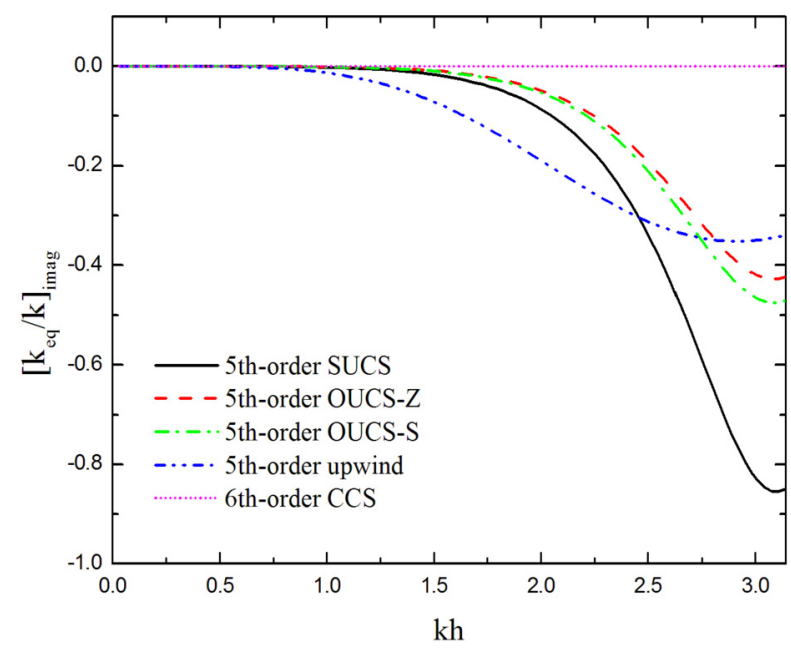

(b)

Fig. 2. (a) Comparison of the real part of $k_{e q} / k$ at the central node in the spectral plane computed by the 5th-order SUCS, OUCS-Z, OUCS-S, upwind and 6th-order CCS schemes. (b) Comparison of the imaginary part of $k_{e q} / k$ at the central node in the spectral plane computed by the 5th-order SUCS, OUCS-Z, OUCS-S, upwind and 6th-order CCS schemes.

is solved in the $0 \leq x \leq 1$ domain with 200 uniformly distributed points. The phase speed is taken as $c=0.05$ such that the initial signal input (as shown in the top panel of Fig. 3a) leaves the computational domain at $t=20$. As in [13], the initial condition has piecewise discontinuity at both the ends and is identically zero outside the domain. With the selected large number of grid points a Nyquist limit on wave number equal to $200 \pi$ is attained. Note that since the equation is linear there is no possibility of aliasing error. The analytical solution is identically zero in the computational domain for $t \geq 20$. A long time Euler explicit time integration with $\Delta t=10^{-4}$ is performed to reveal the stability property of the schemes.

Numerical solutions of the linear wave equation computed by the 5th-order SUCS and 4th-order CCS schemes are plotted in Figs. 3 and compared with the analytical solution. Note that in the bottom panel of Figs. 3 the scales are different and adjusted to the degree of spurious oscillations produced in each scheme after the solution has passed though the domain. It is clearly seen from Fig. 3(a) that the SUCS have sufficient dissipation to suppress the Gibbs' oscillations near the discontinuity. By contrast, the CCS solution leaves numerical oscillations in the domain long after the wave passes though it, as is shown in Fig. 3(b). This is because, being central scheme, the CCS scheme has no dissipation to attenuate the spurious oscillations caused by the discontinuity.

\subsection{Incompressible Navier-Stokes equations and projection procedure}

The governing equations are the unsteady incompressible Navier-Stokes equations which in dimensionless form can be written as

$$
\begin{aligned}
& \frac{\partial \mathbf{u}}{\partial t}=-(\mathbf{u} \cdot \nabla) \mathbf{u}-\nabla p+\frac{1}{R e} \Delta \mathbf{u} \\
& \nabla \cdot \mathbf{u}=0
\end{aligned}
$$

where $\mathbf{u}$ denotes the velocity vector, $p$ is the pressure including any body force that can be written in gradient form (such as gravity), $R e$ is the Reynolds number. The density is already absorbed in the pressure term and is not shown in the above equations.

The following two-step projection method $[18,19]$ is used to solve the governing equations $(10)$ for the whole domain on a staggered rectangular mesh as schematized in Fig. 4, where velocities are located at the cell faces and pressure is located at the cell centers. The first step in the projection method is to introduce an intermediate velocity field $\mathbf{u}^{*}$ calculated from the following relation

$$
\frac{\mathbf{u}^{*}-\mathbf{u}^{n}}{\Delta t}=-\left(\mathbf{u}^{n} \cdot \nabla\right) \mathbf{u}^{n}+\frac{1}{R e} \Delta \mathbf{u}^{n}
$$

where $\mathbf{u}^{n}$ is known at the beginning of a time step. Practically, an alternative form of the momentum equation expressed with velocities postulated at the cell faces is used, written by, 

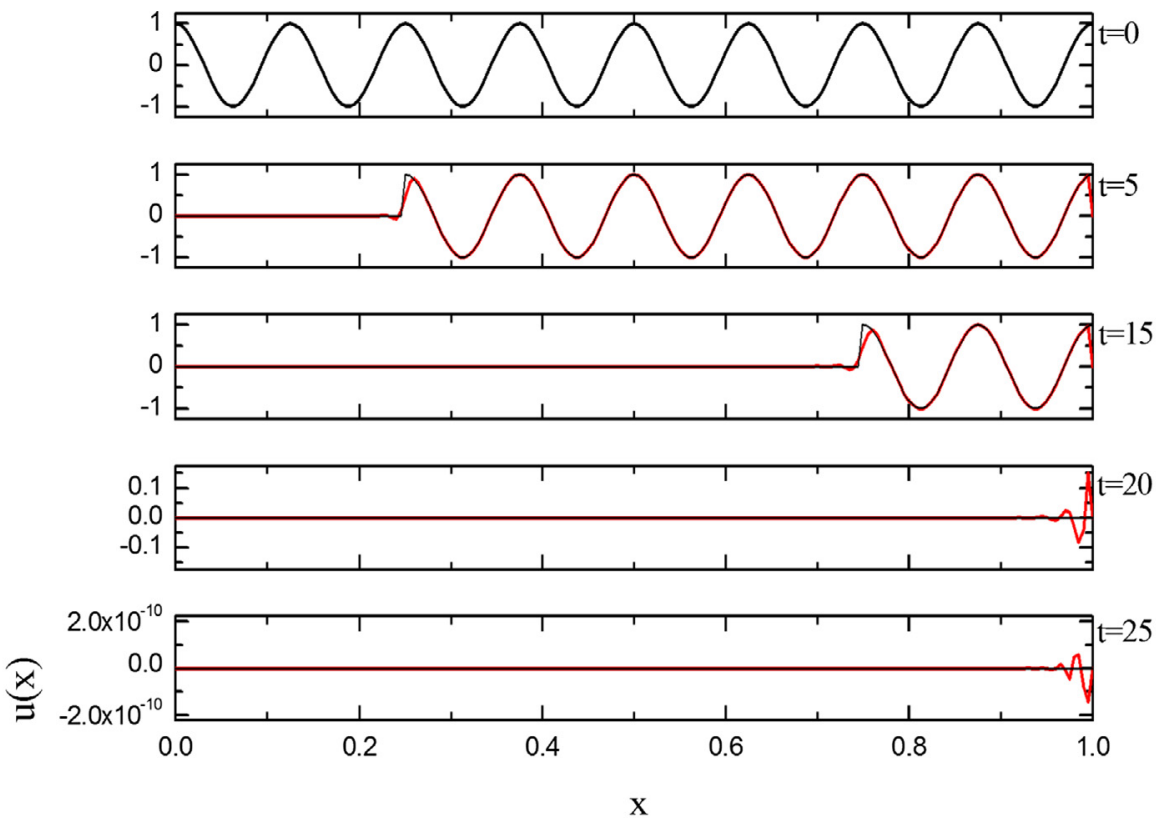

(a)
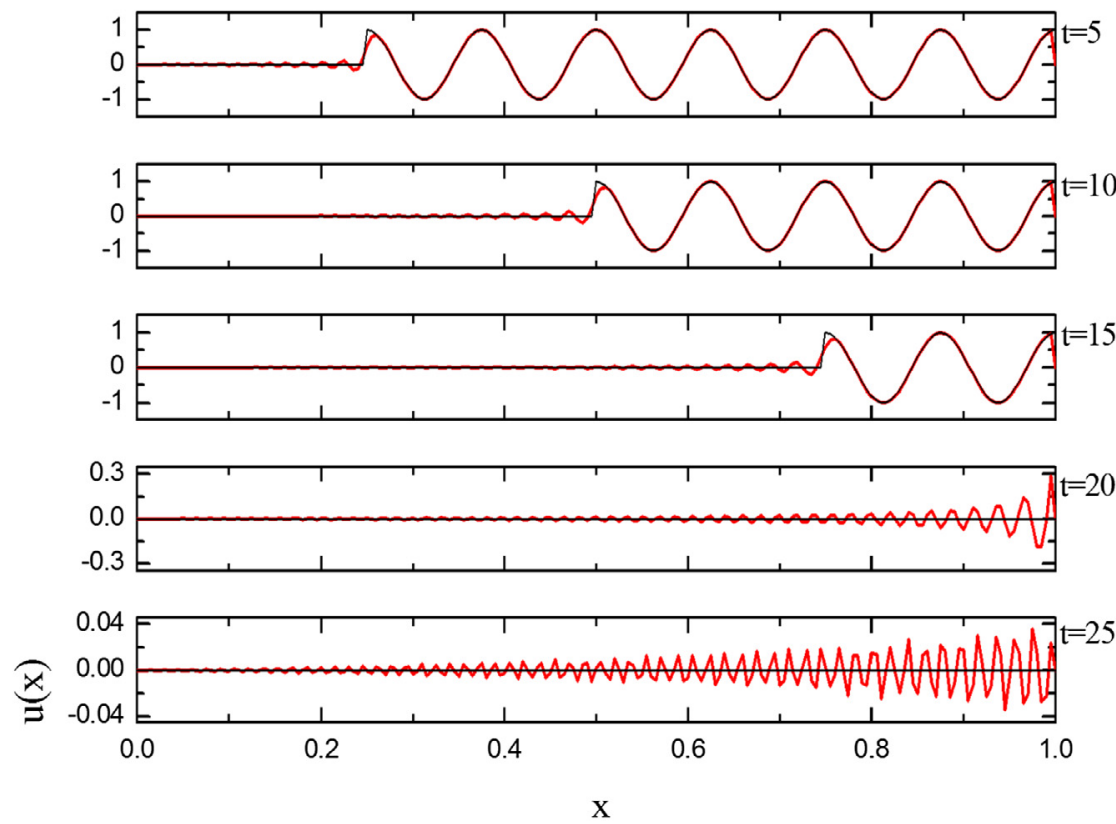

(b)

Fig. 3. Solutions of the linear wave equation computed by the 5th-order SUCS (a) and 4th-order CCS schemes (b) are compared with the analytical solution. The black (thin) solid line and red (thick) solid line denote the analytical and numerical solutions, respectively.

$$
\left\{\begin{array}{l}
\frac{u_{h}^{*}-u_{h}^{n}}{\Delta t}=-u_{h}^{n} \frac{\partial u_{h}^{n}}{\partial x}-v_{h}^{n} \frac{\partial u_{h}^{n}}{\partial y}+\frac{1}{R e}\left(\frac{\partial^{2} u_{h}^{n}}{\partial x^{2}}+\frac{\partial^{2} u_{h}^{n}}{\partial y^{2}}\right) \\
\frac{v_{v}^{*}-v_{v}^{n}}{\Delta t}=-u_{v}^{n} \frac{\partial v_{v}^{n}}{\partial x}-v_{v}^{n} \frac{\partial v_{v}^{n}}{\partial y}+\frac{1}{R e}\left(\frac{\partial^{2} v_{v}^{n}}{\partial x^{2}}+\frac{\partial^{2} v_{v}^{n}}{\partial y^{2}}\right)
\end{array}\right.
$$

in which $u_{h}$ and $v_{h}$ are, respectively, the horizontal and vertical velocity components defined at the cell faces normal to the horizontal $(i, j+1 / 2) ; u_{v}$ and $v_{v}$ are, respectively, the horizontal and vertical velocity components defined at the cell faces normal to the vertical $(i+1 / 2, j)$ (see Fig. 4 ). 


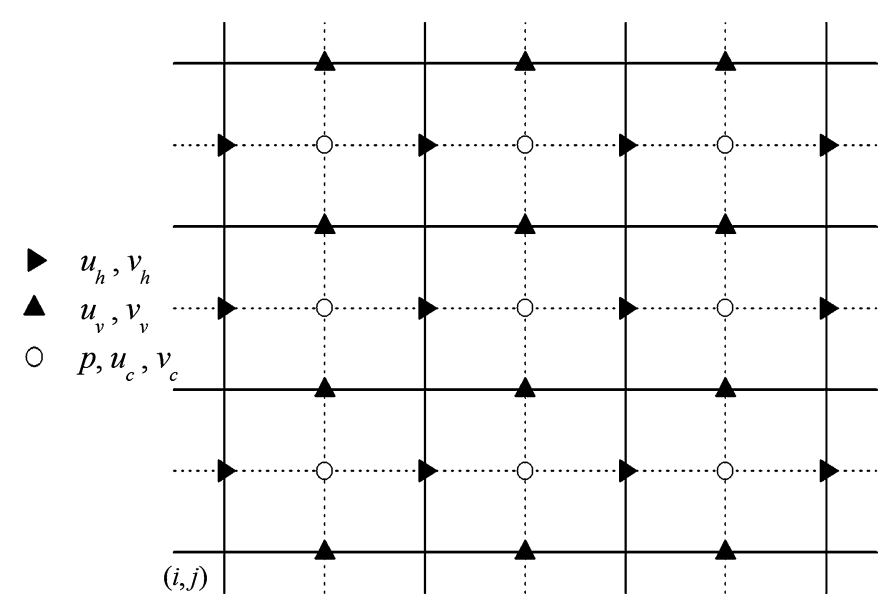

Fig. 4. The staggered grids. Pressure $p$, velocities $u_{c}$ and $v_{c}$ are defined at cell centers $(i+1 / 2, j+1 / 2)$; velocities $u_{h}$ and $v_{h}$ are defined at the cell faces normal to the horizontal $(i, j+1 / 2) ; u_{v}$ and $v_{v}$ are defined at the cell faces normal to the vertical $(i+1 / 2, j)$.

Clearly, the convective terms in the above momentum equations are expressed in the advective form. Note that from solving the above time integration equations one can obtain numerical solutions for $u_{h}$ and $v_{v}$ only. The other two velocity components $u_{v}$ and $v_{h}$ appeared on the RHS of the above equations can be obtained by using interpolation techniques. Specifically, we first evaluate the velocity components $u_{c}$ and $v_{c}$ from interpolating $u_{h}$ and $v_{h}$ at the cell center, respectively. Then, $u_{v}$ and $v_{h}$ can be obtained from interpolating $u_{c}$ and $v_{c}$ at the cell faces normal to the vertical and horizontal, respectively. In this paper, the above interpolation processes are done through using the 4th-order Lagrangian interpolation formulation. After all of the velocity components at the cell faces are given, the first derivatives of $\mathbf{u}$ in the convective terms can thus be evaluated using the left-biased or right-biased SUCS schemes depending on the signs of the variables multiplied to them are positive or negative. The second derivatives of $\mathbf{u}$ contained in the viscous term are evaluated by using the 6th-order CCS scheme.

In the second step, the velocity vector and the pressure are related by

$$
\frac{\mathbf{u}^{n+1}-\mathbf{u}^{*}}{\Delta t}=-\nabla p^{n+1}
$$

Combined with the incompressible constraint condition, the above equation can be reformed to a Poisson equation for solving the pressure variable which is postulated at the cell center, given by

$$
\nabla^{2} p^{n+1}=\frac{1}{\Delta t} \nabla \cdot \mathbf{u}^{*}
$$

As in the classic projection method, both the gradient and Laplacian of $p$ and the divergence of $u^{*}$ in the above two equations are discretized by the 2nd-order central difference scheme. Specifically, the difference scheme for the horizontal component of $\nabla p$ at $(i, j+1 / 2)$ with 2 nd-order of accuracy can be expressed as,

$$
\frac{\partial p}{\partial x}_{i, j+\frac{1}{2}}=\frac{p_{i+\frac{1}{2}, j+\frac{1}{2}}-p_{i-\frac{1}{2}, j+\frac{1}{2}}}{\Delta x}
$$

and the divergence of $\mathbf{u}^{*}$ at $(i+1 / 2, j+1 / 2)$ with 2 nd-order of accuracy can be approximated from

$$
\nabla \cdot \mathbf{u}_{i+\frac{1}{2}, j+\frac{1}{2}}^{*}=\frac{u_{i+1, j+\frac{1}{2}}^{*}-u_{i, j+\frac{1}{2}}^{*}}{\Delta x}+\frac{v_{i+\frac{1}{2}, j+1}^{*}-v_{i+\frac{1}{2}, j}^{*}}{\Delta y}
$$

At an inner grid center $(i+1 / 2, j+1 / 2)$, the second $y$-derivative (for instance) of $p$ can be discretized as

$$
{\frac{\partial^{2} p}{\partial y^{2}}}_{i+\frac{1}{2}, j+\frac{1}{2}}=\frac{p_{i+\frac{1}{2}, j+\frac{3}{2}}+p_{i+\frac{1}{2}, j-\frac{1}{2}}-2 p_{i+\frac{1}{2}, j+\frac{1}{2}}}{\Delta y^{2}}
$$

To solve the pressure Poisson equation (PPE) (14), all of the pressure variables in the interior of the domain are unknown and to be determined from solving a large sparse linear system

$$
\left\{a_{l, l}\right\}_{(M \times N) \times(M \times N)}\left\{p_{l}\right\}_{M \times N}=\left\{b_{l}\right\}_{M \times N}
$$


where $M$ and $N$ are, respectively, the total grid numbers in the horizontal and vertical directions of a rectangular domain. For an interior grid center $(i+1 / 2, j+1 / 2), 0<i<M-1,0<j<N-1$, and the second-order accuracy requirement, the number of elements in the $l$ th row $(l=i \times N+j)$ of this system is 5 , and the coefficients are given by

$$
\begin{aligned}
& a_{l-N, l}=a_{l+N, l}=\frac{1}{\Delta x^{2}}, \quad a_{l-1, l}=a_{l+1, l}=\frac{1}{\Delta y^{2}}, \quad a_{l, l}=-\frac{2}{\Delta x^{2}}-\frac{2}{\Delta y^{2}}, \\
& b_{l}=\frac{1}{\Delta t}\left(\nabla \cdot \mathbf{u}^{*}\right)_{i+\frac{1}{2}, j+\frac{1}{2}}
\end{aligned}
$$

Furthermore, to close this system and make this system solvable, values or gradient values of $p$ on the boundaries should be given as known variables (which should be relegated to the right hand side of the linear system). If an open boundary problem is studied, the values of $p$ on the boundary should be given as known variables, while, for solid boundary problems, the gradient values of $p$ on the boundary are usually used to close the linear system of $p$. Considering a solid boundary grid, for example, $(1 / 2, j+1 / 2), 0<j<N-1$, the discretization of the second $x$-derivative of $p$ can be written as

$$
{\frac{\partial^{2} p}{\partial x^{2} \frac{1}{2}, j+\frac{1}{2}}}=\frac{1}{\Delta x}\left[\frac{p_{\frac{3}{2}, j+\frac{1}{2}}-p_{\frac{1}{2}, j+\frac{1}{2}}}{\Delta x}-\left(\frac{\partial p}{\partial x}\right)_{0, j+\frac{1}{2}}\right]
$$

Therefore, the number of elements in the $j$ th row of the system reduces to 4 , and the coefficients of the system are given by

$$
\begin{aligned}
& a_{j+N, j}=\frac{1}{\Delta x^{2}}, \quad a_{j-1, j}=a_{j+1, j}=\frac{1}{\Delta y^{2}}, \quad a_{j, j}=-\frac{1}{\Delta x^{2}}-\frac{2}{\Delta y^{2}}, \\
& b_{j}=\frac{1}{\Delta t}\left(\nabla \cdot \mathbf{u}^{*}\right)_{\frac{1}{2}, j+\frac{1}{2}}+\frac{1}{\Delta x}\left(\frac{\partial p}{\partial x}\right)_{0, j+\frac{1}{2}}
\end{aligned}
$$

Note that in the above equations the gradient values of $p$ at location $(0, j+1 / 2)$ denoted by $(\boldsymbol{\partial} \boldsymbol{p} / \boldsymbol{\partial x})_{\mathbf{0}}, \boldsymbol{j}+\mathbf{1} / \mathbf{2}$ should be postulated as known variables. To acquire the gradient values of $p$ on the solid boundaries, the following Neumann boundary condition derived from the momentum equation is usually applied [20-24]

$$
\left.\mathbf{n} \cdot \nabla p\right|_{\partial \Omega}=\mathbf{n} \cdot\left[-\frac{\partial \mathbf{u}}{\partial t}-(\mathbf{u} \cdot \nabla) \mathbf{u}+\frac{1}{\operatorname{Re}} \Delta \mathbf{u}\right]
$$

where $\mathbf{n}$ denotes the unit outer normal vector on the boundary. Since all of the solid boundaries considered in this paper are at rest, the above formulation can be further simplified to

$$
\left.\mathbf{n} \cdot \nabla p\right|_{\partial \Omega}=\frac{1}{R e} \mathbf{n} \cdot \Delta \mathbf{u}
$$

The above equation means that the gradient values of $p$ on the solid boundary can be determined and substituted by the value of normal component of the viscous term on the boundary. Here, the second derivatives of $\mathbf{u}$ involved in the viscous term on the boundary $\partial \Omega$ are evaluated by using the 4th-order explicit difference scheme. After the term on the right hand side of (19) is evaluated, the gradient values of $p$ on the solid boundary are then known and the linear system of $p$ for the whole domain is closed and can thus be solved.

It should be remarked particularly that to adequately solve the pressure Poisson equation (PPE) (14) the boundary condition for the intermediate velocity $\mathbf{u}^{*}$ should also be specified. It is clear from Eq. (13) that the boundary condition of the $\mathbf{u}^{*}$ on a solid boundary is equal to

$$
\left(\mathbf{u}^{*}\right)_{\partial \Omega}=\Delta t\left(\nabla p^{n+1}\right)_{\partial \Omega}
$$

If the divergence of $\mathbf{u}^{*}$ in Eq. (17) is discretized with a second-order accuracy, $b_{j}$ can be rewritten into the following form

$$
b_{j}=\frac{u_{1, j+\frac{1}{2}}^{*}-u_{0, j+\frac{1}{2}}^{*}}{\Delta t \Delta x}+\frac{v_{\frac{1}{2}, j+1}^{*}-v_{\frac{1}{2}, j}^{*}}{\Delta t \Delta y}+\frac{1}{\Delta x}\left(\frac{\partial p}{\partial x}\right)_{0, j+\frac{1}{2}}
$$

After substituting the following boundary condition

$$
u_{0, j+\frac{1}{2}}^{*}=\Delta t\left(\frac{\partial p}{\partial x}\right)_{0, j+\frac{1}{2}}
$$

into the expression of $b_{j}$, one can have immediately that

$$
b_{j}=\frac{u_{1, j+\frac{1}{2}}^{*}}{\Delta t \Delta x}+\frac{v_{\frac{1}{2}, j+1}^{*}-v_{\frac{1}{2}, j}^{*}}{\Delta t \Delta y}
$$


Clearly, both the boundary conditions for $\mathbf{u}^{*}$ and $\nabla p$ are eliminated from the above equation. According to this observation, the following vanishing boundary conditions for $\mathbf{u}^{*}$ and $\nabla p$ is thus chosen practically in our simulations

$$
\left(\mathbf{u}^{*}\right)_{\partial \Omega}=\Delta t\left(\nabla p^{n+1}\right)_{\partial \Omega}=0
$$

It should be noted additionally that if the divergence of $\mathbf{u}^{*}$ in Eq. (17) is discretized with a higher order (for example, 4th-order) accuracy, the boundary conditions for $\mathbf{u}^{*}$ and $\nabla p$ will not be eliminated simultaneously from the expression of $b_{j}$ any more and the boundary condition for $\nabla p$ defined by Eq. (19) will be useful under this situation. This topic can be discussed thoroughly in another study.

In choosing boundary schemes to evaluate derivatives of $\mathbf{u}$, it is found that there is no significant difference between using the 4th-order explicit and implicit boundary schemes. Therefore, the 4th-order explicit boundary schemes are selected typically for approximating first and second derivatives of $\mathbf{u}$ at the solid boundary points and near-boundary points. The explicit forms of these schemes are

$$
\begin{aligned}
& u_{0}^{\prime}=\frac{ \pm 1}{12 h}\left(-25 u_{0}+48 u_{ \pm 1}-36 u_{ \pm 2}+16 u_{ \pm 3}-3 u_{ \pm 4}\right) \\
& u_{ \pm 1}^{\prime}=\frac{ \pm 1}{12 h}\left(-3 u_{0}-10 u_{ \pm 1}+18 u_{ \pm 2}-6 u_{ \pm 3}+u_{ \pm 4}\right) \\
& u_{0}^{\prime \prime}=\frac{1}{12 h^{2}}\left(45 u_{0}-154 u_{ \pm 1}+214 u_{ \pm 2}-156 u_{ \pm 3}+61 u_{ \pm 4}-10 u_{ \pm 5}\right) ; \\
& u_{ \pm 1}^{\prime \prime}=\frac{1}{12 h^{2}}\left(10 u_{0}-15 u_{ \pm 1}-4 u_{ \pm 2}+14 u_{ \pm 3}-6 u_{ \pm 4}+u_{ \pm 5}\right)
\end{aligned}
$$

in which the positive (negative) signs are taken if the left (right) boundary is considered.

To enhance the accuracy in time, high order Runge-Kutta method can be taken into consideration. For instance, the second-order Runge-Kutta method can be implemented via

$$
\begin{aligned}
& \mathbf{u}^{(1)}=\mathbf{u}^{n}+\Delta t \cdot L_{u}\left(\mathbf{u}^{n}\right) \\
& \mathbf{u}^{n+1}=\frac{1}{2} \mathbf{u}^{n}+\frac{1}{2} \Delta t \cdot L_{u}\left(\mathbf{u}^{(1)}\right)
\end{aligned}
$$

in which $L_{u}(\cdot)$ represents the operator for evaluating $\mathbf{u}^{n+1}$ from $\mathbf{u}^{n}$ in (11)-(14). To acquire stable numerical solutions a $\mathrm{CFL}=0.4$ condition is used.

\subsection{Lid-driven cavity flow}

In this section, we choose the most classical and celebrated lid-driven cavity flow to test the newly developed SUCS schemes. The flow inside a lid-driven cavity constitutes a classical benchmark problem which has been widely used for the assessment of numerical methods and the validation of Navier-Stokes solvers [25,10,11,26].

For this problem, the normal and tangential velocity boundary conditions are imposed at each boundary of a square domain $[0,1] \times[0,1]$. The boundary conditions are those of no slip: on the upper wall $u=1$ and $v=0$, while on all other walls $u=v=0$.

For steady solutions to the lid-driven cavity problem many benchmark datasets exist over a broad range of Reynolds numbers. While there are many papers on this topic, we refer to the oft quoted results in [27] to compare our results obtained using SUCS schemes. In Figs. 5 the stream function contours are plotted for Reynolds number $R e=5000$ and 7500 by using the 5th-order SUCS scheme and a uniform grid with $(128 \times 128)$ points. In Figs. 6 the $x$ - and $y$-components of velocity along, respectively, the vertical and horizontal central lines are presented and compared with the classic results. It is clear that our numerical results are in good agreement with the benchmark values.

To further identify distinctions between the high order schemes, more of our attentions are paid on the high Reynolds number cases. This is because with the increase of the Reynolds number, the flows in the lid-driven cavity exhibit many complex and unique vortex patterns. Several secondary and tertiary vortices with characteristics depending on $R e$ can be observed. These special flow configurations have attracted long-term interests of the researches. For example, some pioneer and recent works $[27,28]$ have acquired stable steady solutions until $R e=10,000$. Furthermore, at a Reynolds number between 7500 and 8000 a Hopf bifurcation was identified and the time-periodic solutions for Reynolds number higher than this critical value were obtained, for example, by Auteri et al. [29] and Bruneau and Saad [30]. The major work of this paper is to scrutinize the global (local) time periodic characteristics of the flows for $R e=10,000(20,000)$ and identify the unique vortex structures that can be revealed by using the finer grids systems and the high resolution numerical schemes.

In Figs. 7 full cycles of the stream function contours obtained by using the 5th-order SUCS scheme and a uniform grid of size $(64 \times 64)$ is shown for $R e=10,000$. The periodic feature can be evidenced obviously from comparing the similarities between (a), (e) and (f). Thus, the averaged time period can be approximated by $(4.25-0) / 2=2.13$ s. Note that the initial state depicted by Fig. 7(a) should be obtained after a long-time continuous computation from the static state. However, it is not necessary to provide the accurate computation time that has been spent to arrive at this initial state. This is 


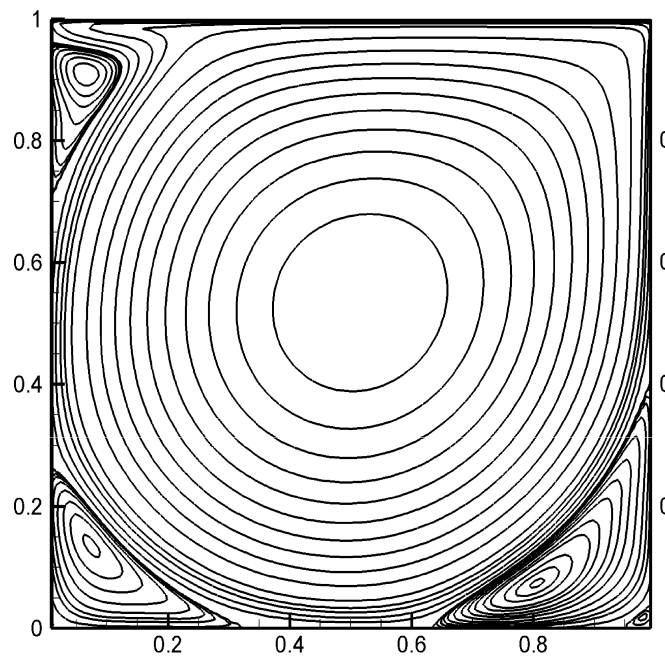

(a)

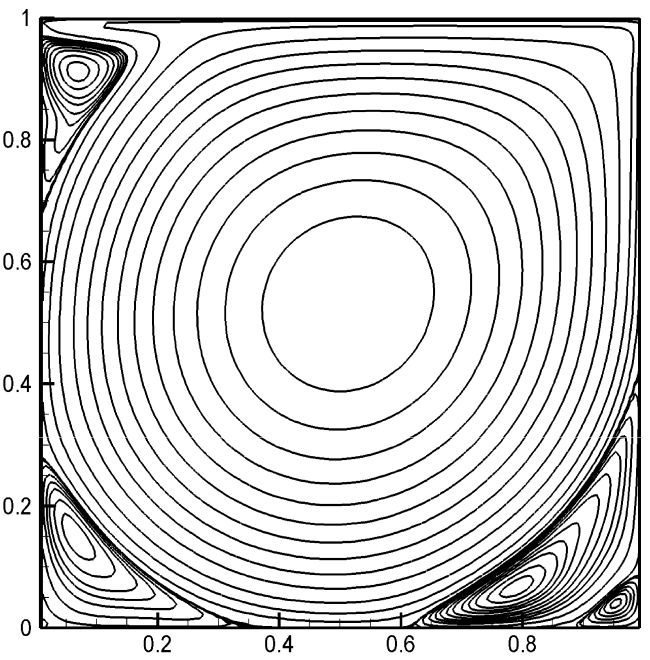

(b)

Fig. 5. Stream function contours for the lid-driven cavity problem upon using the 5th-order SUCS scheme and a grid of $(128 \times 128)$. The Reynolds numbers are $R e=5000$ (a) and $R e=7500$ (b), respectively.

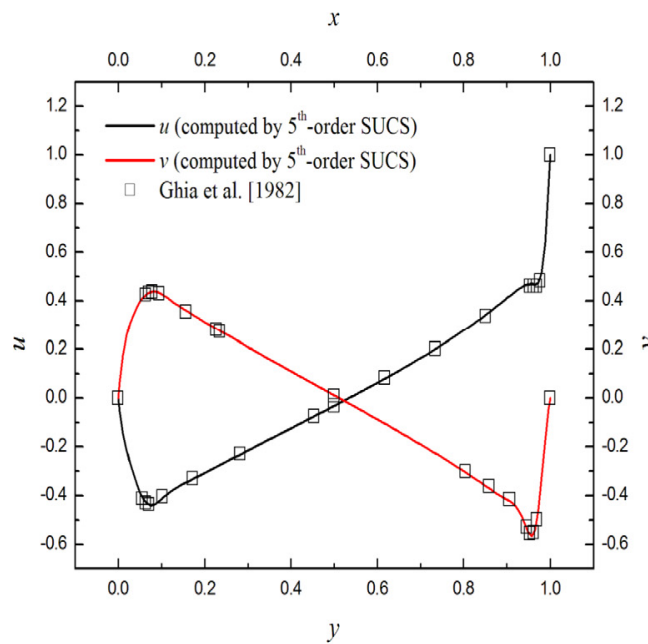

(a)

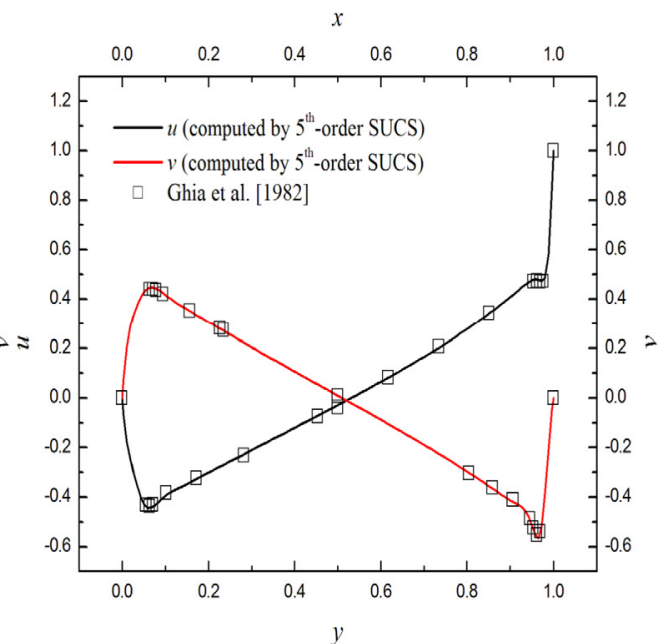

(b)

Fig. 6. Comparison of $u$-velocity ( $v$-velocity) along the vertical (horizontal) central line of the lid-driven cavity with the benchmark solutions obtained at $\operatorname{Re}=5000$ (a) and $R e=7500$ (b).

because practically in order to save the computation time the computation was started not directly at the static state with $R e=10,000$ but using the obtained steady results of lower Reynolds number such as $R e=7500$ as the initial condition. Therefore, we can provide merely an approximate computation time cost to explain how long the given initial state is achieved from the static state which is over $1000 \mathrm{~s}$. On the other hand, since this paper cares chiefly about the periodic characteristics of the flow, we set $t=0 \mathrm{~s}$ to illustrate the initial state figured by Fig. 7(a) and to facilitate grasping the time period of this process.

Figs. 8 present full cycles of the stream function contours obtained by using the 5th-order SUCS scheme and a uniform grid of size $(128 \times 128)$ for $R e=10,000$. The initial state represented by Fig. $8(\mathrm{a})$ is obtained after a continuous computation from the static state has been run for over $1000 \mathrm{~s}$. The periodic feature is also evident and can be identified from comparing the similarities between (a), (f), ( $\mathrm{g}$ ) and (h). The averaged time period of this case can be computed approximately to be $(4.75-0) / 2=2.38 \mathrm{~s}$ by comparing (a) and $(\mathrm{g})$ or to be $(7-0) / 3=2.33 \mathrm{~s}$ by comparing $(\mathrm{a})$ and $(\mathrm{h})$. It may be noted that similar periodic features of the flow field were presented in [10] and [30], while, the grids used in these two references were $(256 \times 256)$ and $(1024 \times 1024)$, respectively. Moreover, it can be found in the following part of this paper that if a 


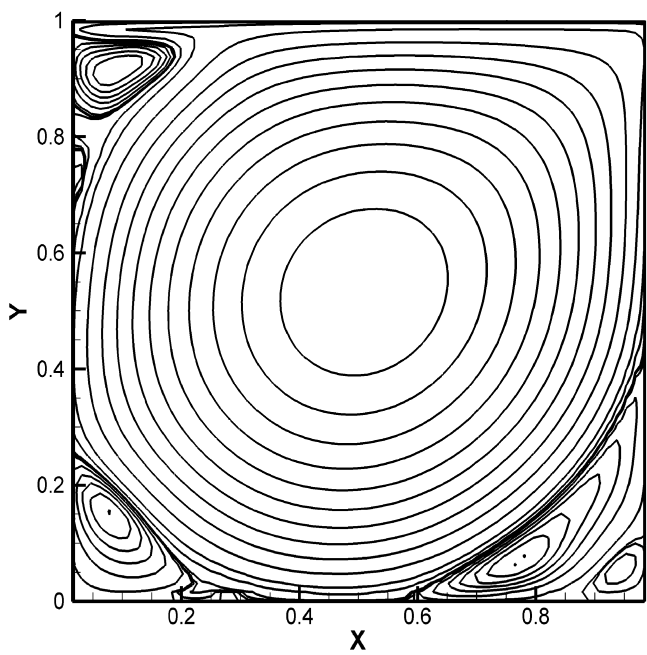

(a) $t=0 \mathrm{~s}$

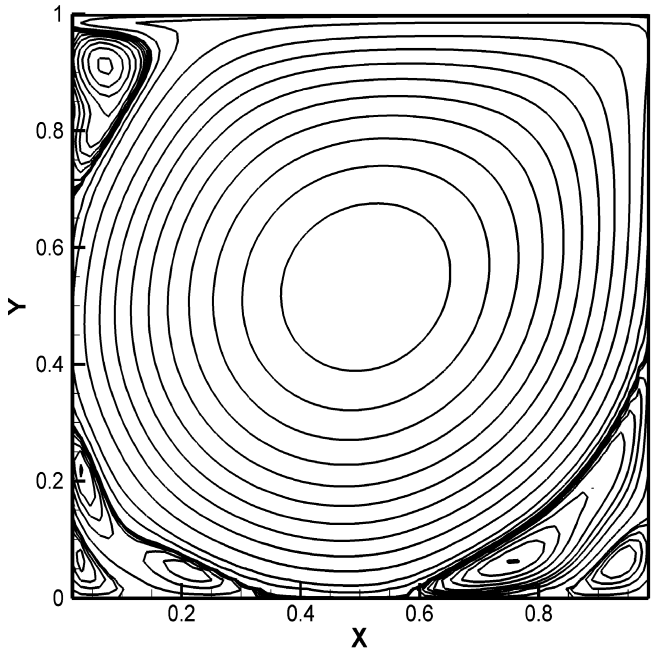

(c) $t=1 \mathrm{~s}$

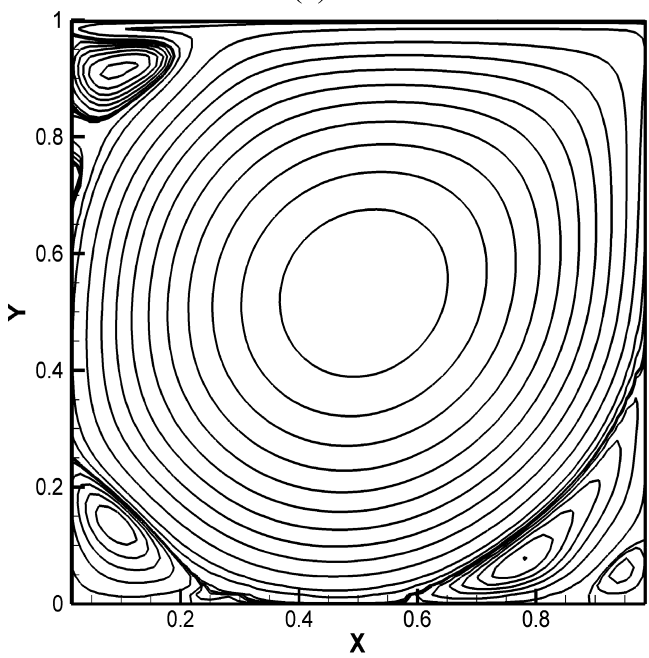

(e) $t=2 \mathrm{~s}$

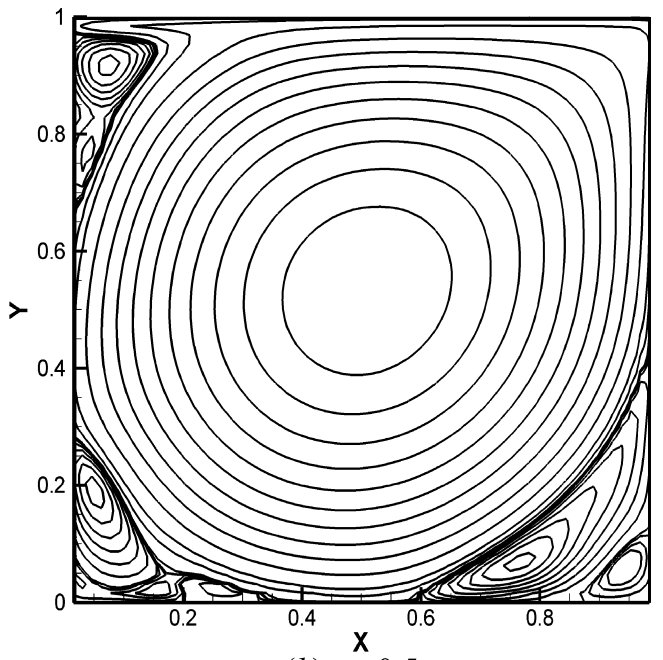

(b) $t=0.5 \mathrm{~s}$

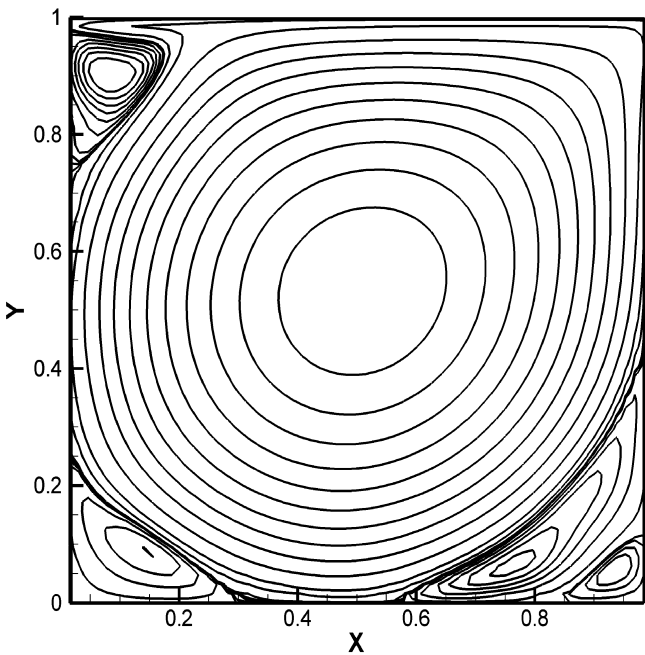

(d) $t=1.5 \mathrm{~s}$

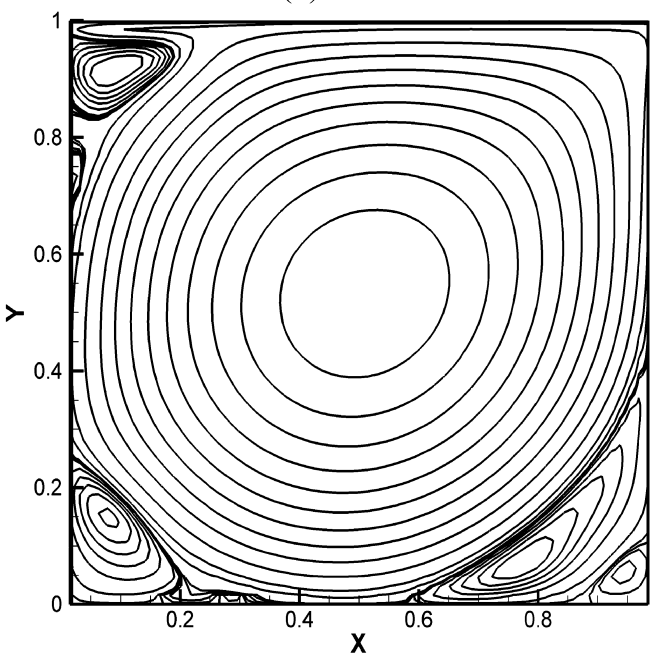

(f) $t=4.25 \mathrm{~s}$

Fig. 7. Full cycles of the stream function contours of the flow in a lid-driven cavity for Re $=10,000$ solved using the 5 th-order SUCS scheme and a uniform grid of size $(64 \times 64)$. The initial state described by Fig. $7(\mathrm{a})$ is achieved after a continuous computation from the static state has been run for over 1000 s. 


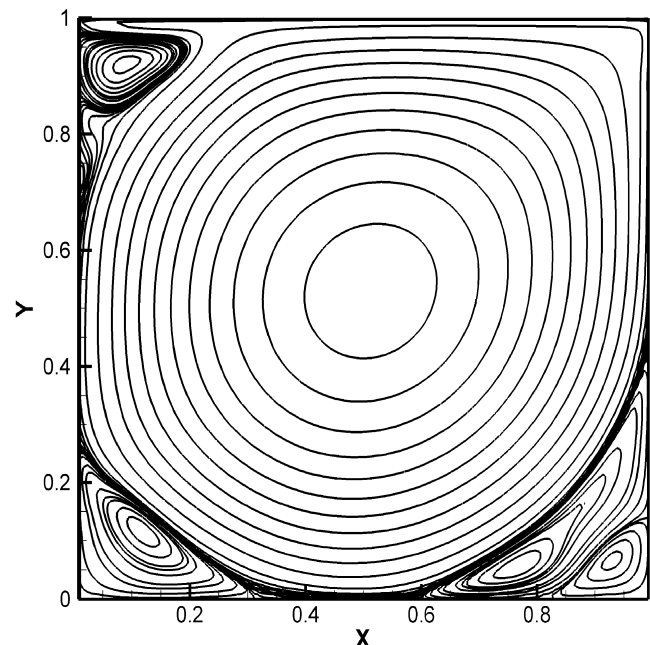

(a) $t=0 \mathrm{~s}$

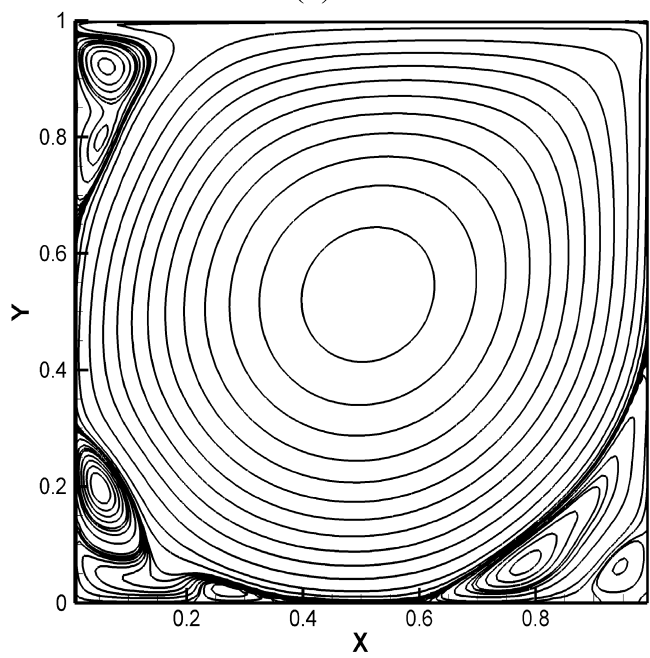

(c) $t=1 \mathrm{~s}$

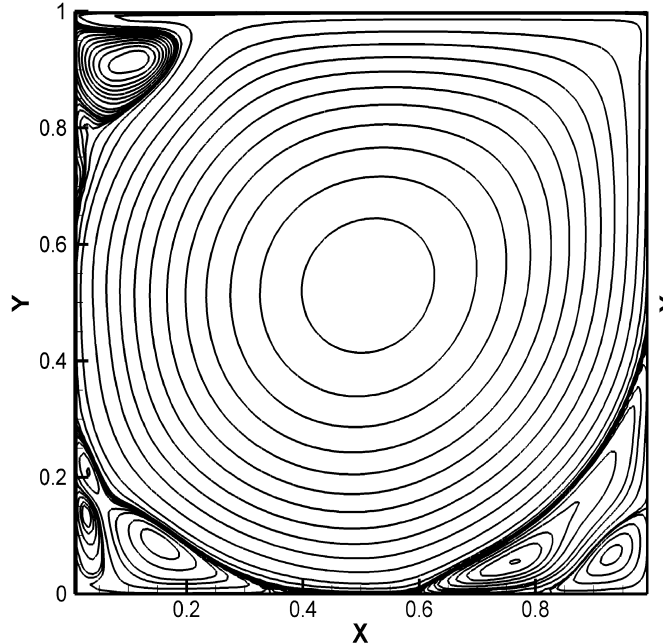

(e) $t=2 \mathrm{~s}$

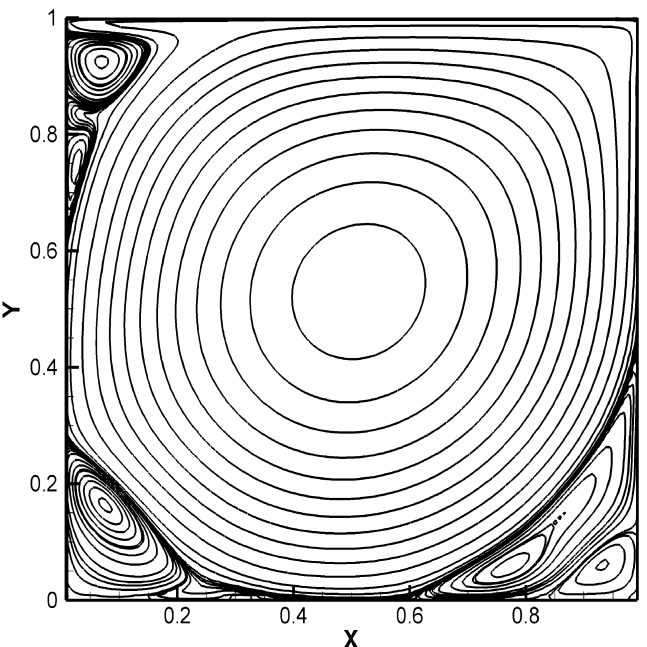

(b) $t=0.5 \mathrm{~s}$

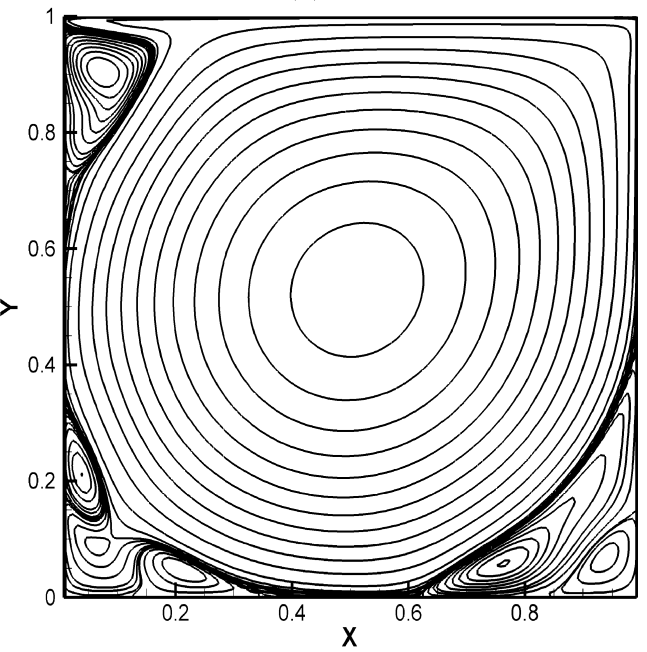

(d) $t=1.5 \mathrm{~s}$

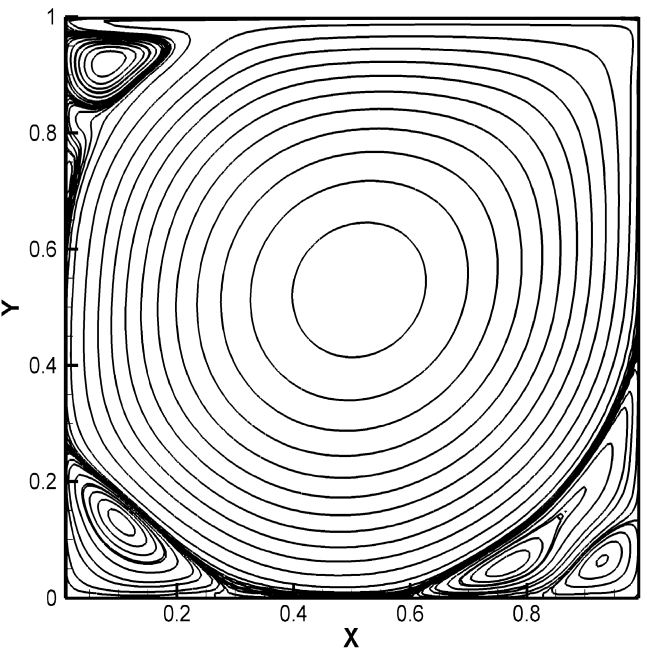

(f) $t=2.5 \mathrm{~s}$

Fig. 8. Full cycles of the stream function contours of the flow in a lid-driven cavity for $R e=10,000$ solved using the 5 th-order SUCS scheme and a uniform grid of size $(128 \times 128)$. The initial state described by Fig. $8(\mathrm{a})$ is achieved after a continuous computation from the static state has been run for over $1000 \mathrm{~s}$. 


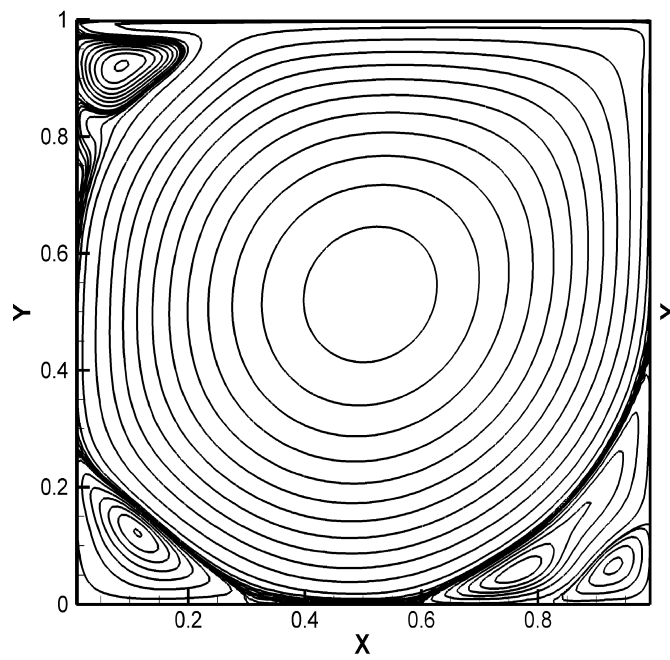

(g) $t=4.75 \mathrm{~s}$

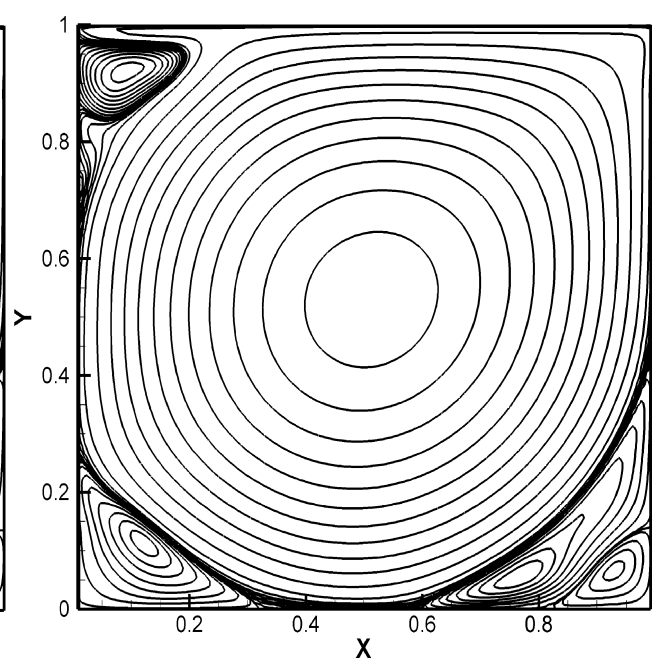

(h) $t=7 \mathrm{~s}$

Fig. 8. (continued)

finer mesh $(256 \times 256)$ is used more vortex structures can be captured. This different result provides an evidence for the high accuracy of the scheme used in this paper compared with those used in the mentioned literatures.

From comparing Figs. 7 and 8, the grid convergence of the computation is demonstrated apparently. The flow fields in these two figures are similar except in Figs. 8 the streamlines are more smooth. Furthermore, there is a small difference between the time periods of the two cases. This difference may be largely ascribed to that under the finer grid $(128 \times 128)$ more minor flow processes have been captured which are not evidently exhibited but have altered the time period of the flow.

The above inference can be further substantiated by running more simulations under a finer mesh. Figs. 9 and 10 present two consecutive cycles of the stream function contours obtained by using the 5th-order SUCS scheme and a uniform grid of size $(256 \times 256)$ for $R e=10,000$. The initial state denoted by Fig. 9(a) is obtained after a continuous computation from the static state has been run for over $1000 \mathrm{~s}$. The periodic feature in Figs. 9 can be identified from comparing the similarities between Figs. 9(a) and (o) and between Figs. 9(b) and (p). From comparing Figs. 9(a) and (o) it can be inferred that the time period is $7 \mathrm{~s}$ which is significantly longer than those indicated from Figs. 7 and 8 . This longer time period can be attributed to that a new sub-process represented by V2 in Fig. 9(h) is captured from using this finer grid. The other process that is denoted by V1 and shown in Figs. 9(c) and (1) resembles closely the result shown in Fig. 8(c). Two implications can thus be revealed from these results. First, the common V1 structures obtained in Figs. 8 and 9 manifest the grid convergence of the scheme. Second, the unique V2 structure portrayed in Figs. 9 exhibits the advantage of using finer grids.

To consolidate the periodic nature of the flow, more numerical results are presented. Figs. 10 present another cycle of the stream function contours subsequent to that shown in Figs. 9. The periodic feature in Figs. 10 can be found from comparing the similarities between Fig. 9(o) and Fig. 10(v) and between Fig. 9(p) and Fig. 10(w). The time period in Figs. 10 can then be approximated by $18.5-7=11.5 \mathrm{~s}$, different considerably to the time period found in Figs. 9. The reason for the different time periods may be due to the random and unpredictable emergence of the sub-process V2 shown in Fig. 10(f).

In summary, the numerical results shown in Figs. 7, 8, 9 and 10 have given sufficient demonstrations for the grid convergence of the SUCS scheme. The advantage of refining the grids is manifested by the appearance of the new sub-process of the vortexes which cannot be captured when a coarse grid is used.

Although the grid convergence of the scheme has been demonstrated successfully, it is still hard to examine order convergence for the new schemes. This is understandable by noting that the lowest order of accuracy is dominated by the accuracy of the solution of the pressure Poisson equation. Therefore, to properly analyze the order convergence for the new SUCS scheme, a model that can acquire higher order solutions for the pressure Poisson equation must be used concurrently. This can be done in another study.

In addition to the oft simulated case with $R e=10,000$, we also examine a higher Reynolds number case with $R e=20,000$ under which more unique flow patterns with secondary and tertiary vortices at the corner of the cavity can be observed. In the following results, it can be seen that the results for $R e=20,000$ are quite different to those for $R e=10,000$. No evident global periodic features can be grasped even if a rather coarse grid $(64 \times 64)$ is used. As has been shown previously, for the case of $R e=10,000$ the small scale sub-processes of the flow are filtered out and hence large scale periodic feature can be retained when a coarse grid is used. However, for $R e=20,000$ the flow is always in an aperiodic state even if a coarse grid of size $(64 \times 64)$ is used which manifests the unique nature of the higher Reynolds number flows in a lid-driven cavity. 


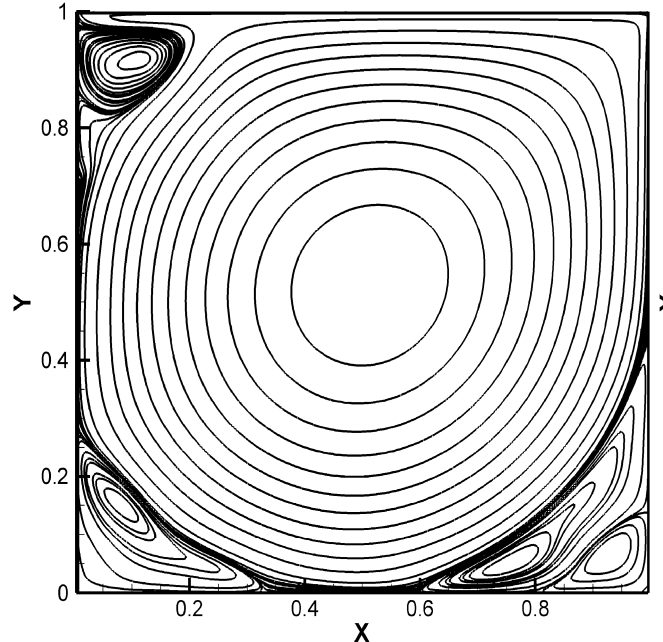

(a) $t=0 \mathrm{~s}$

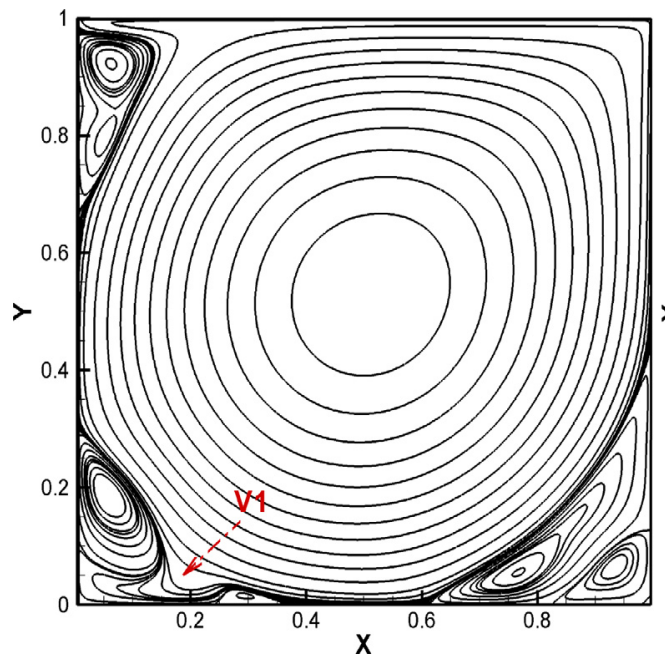

(c) $t=1 \mathrm{~s}$

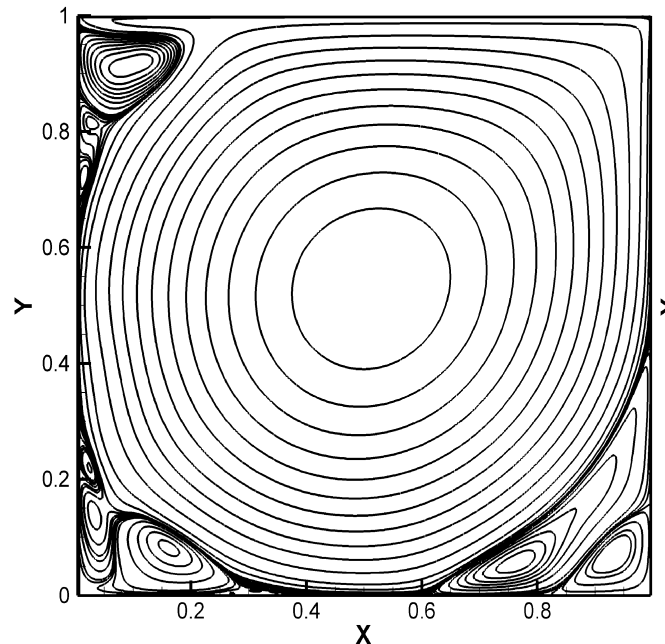

(e) $t=2 \mathrm{~s}$

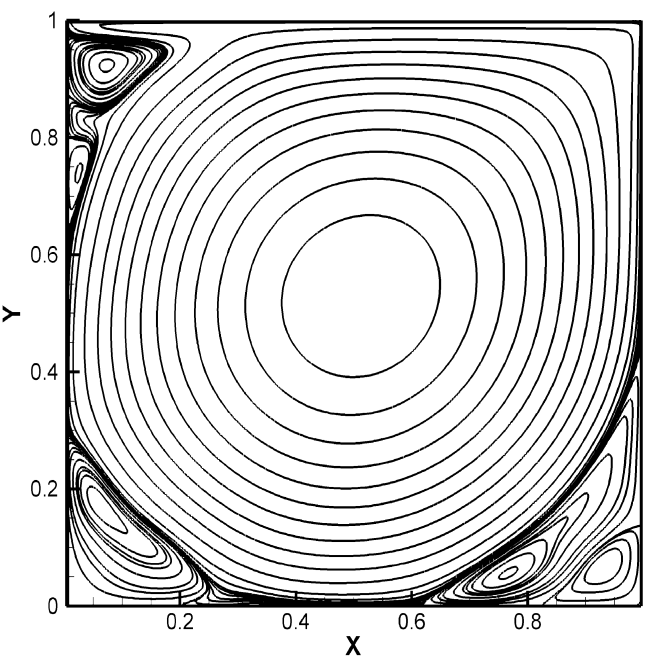

(b) $t=0.5 \mathrm{~s}$

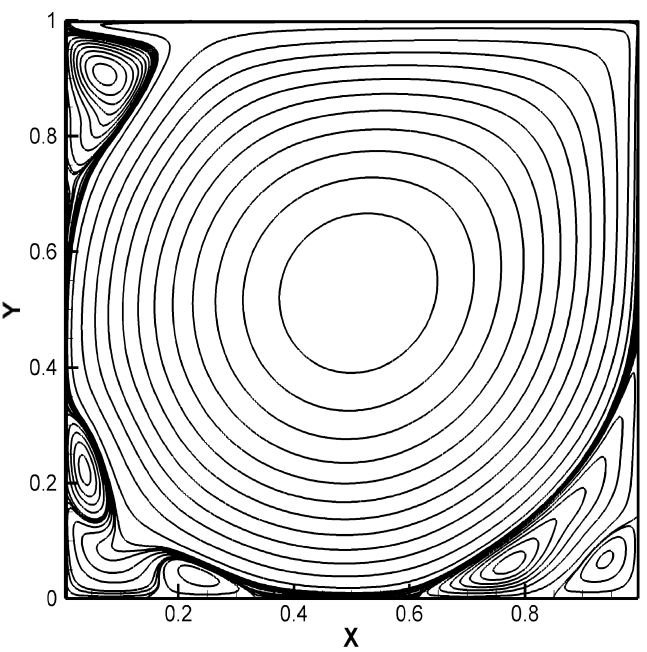

(d) $t=1.5 \mathrm{~s}$

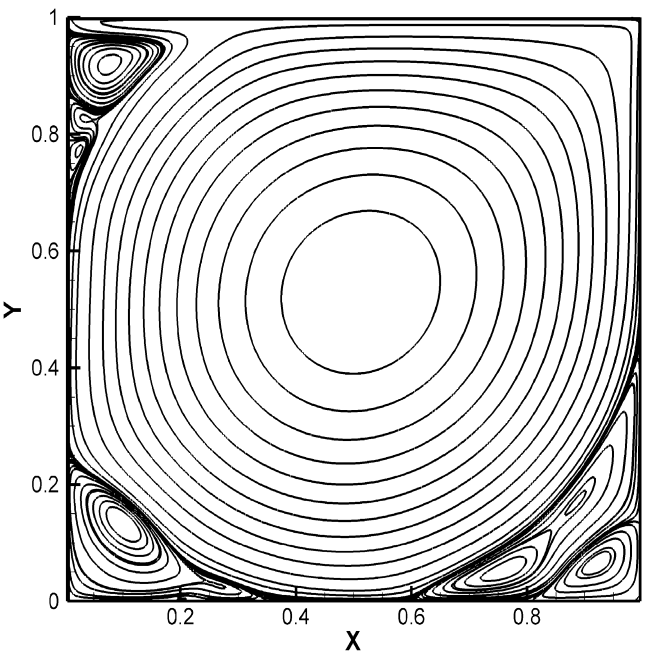

(f) $t=2.5 \mathrm{~s}$

Fig. 9. A full cycle of the stream function contours of the flow in a lid-driven cavity for Re $=10,000$ solved using the 5 th-order SUCS scheme and a uniform grid of size $(256 \times 256)$. The initial state described by Fig. 9(a) is achieved after a continuous computation from the static state has been run for over $1000 \mathrm{~s}$. 


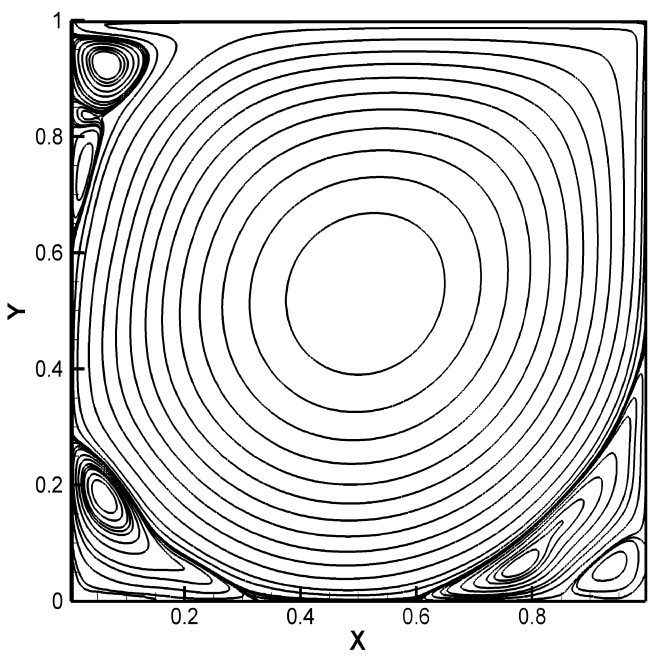

(g) $t=3 \mathrm{~s}$

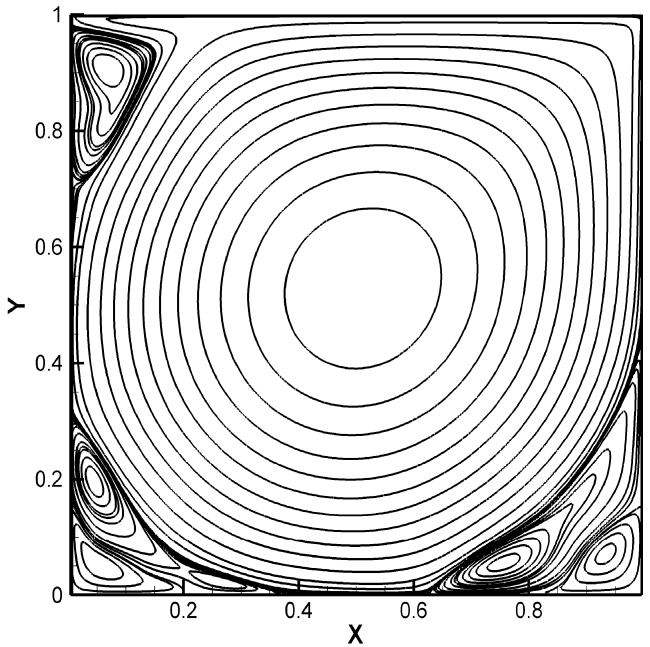

(i) $t=3.75 \mathrm{~s}$

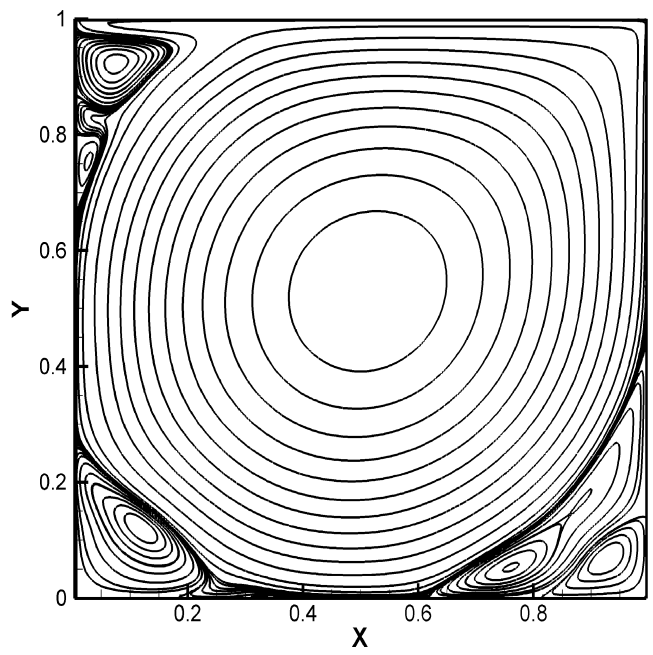

(k) $t=5 \mathrm{~s}$

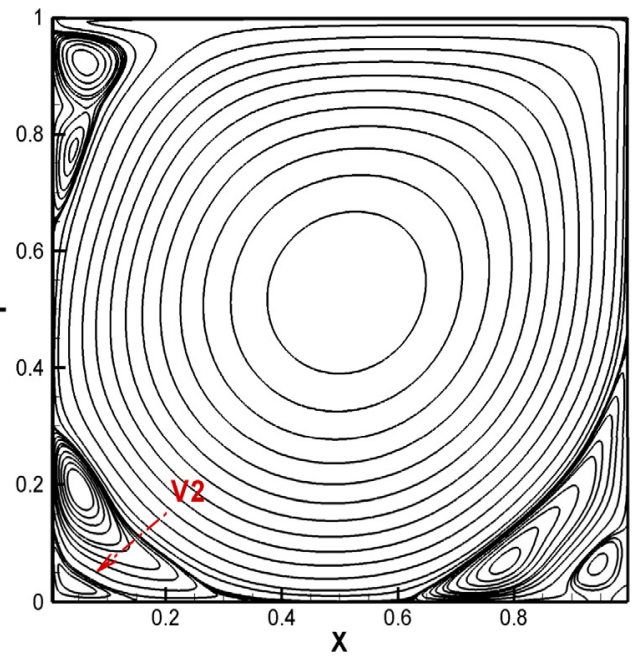

(h) $t=3.25 \mathrm{~s}$

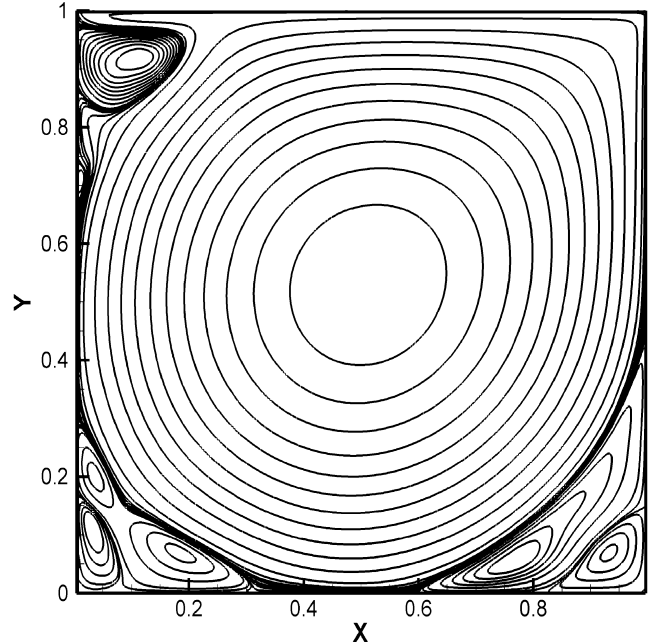

(j) $t=4.5 \mathrm{~s}$

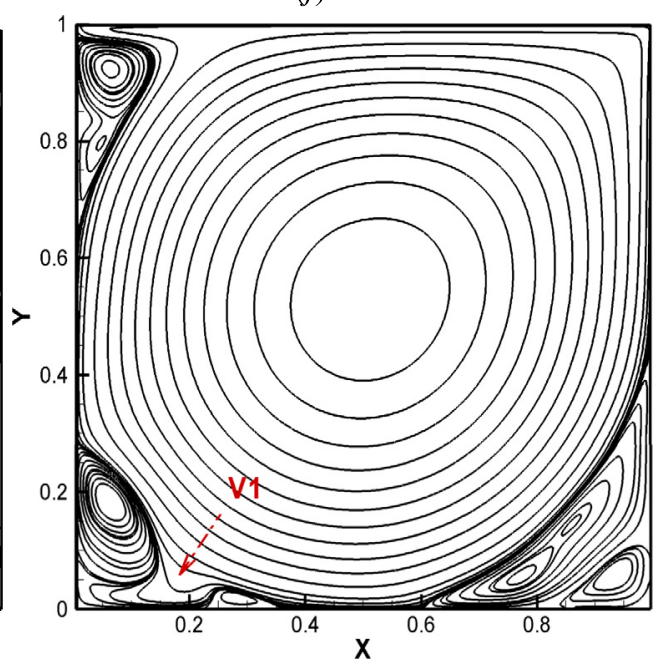

(l) $t=5.5 \mathrm{~s}$

Fig. 9. (continued) 


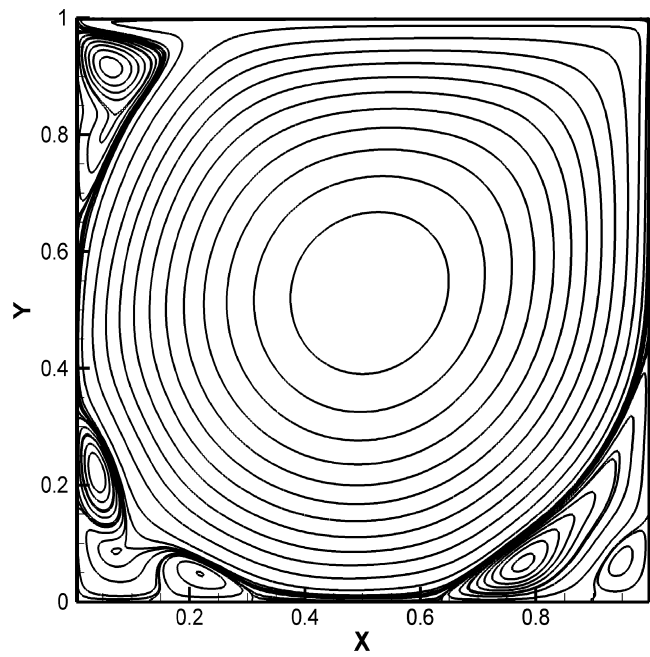

(m) $t=6 \mathrm{~s}$

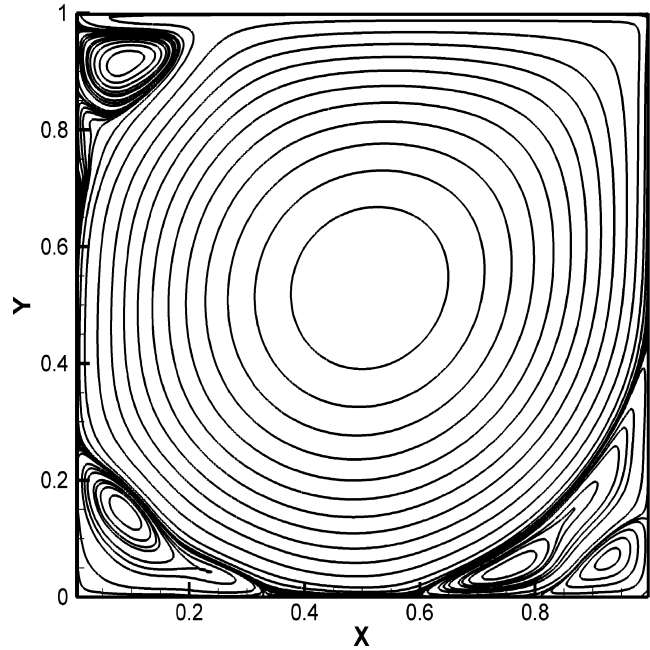

(o) $t=7 \mathrm{~s}$

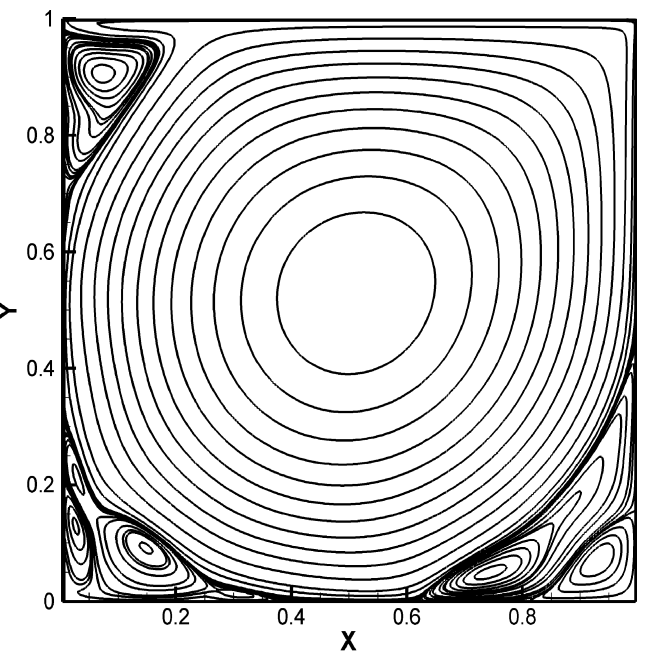

(n) $t=6.5 \mathrm{~s}$

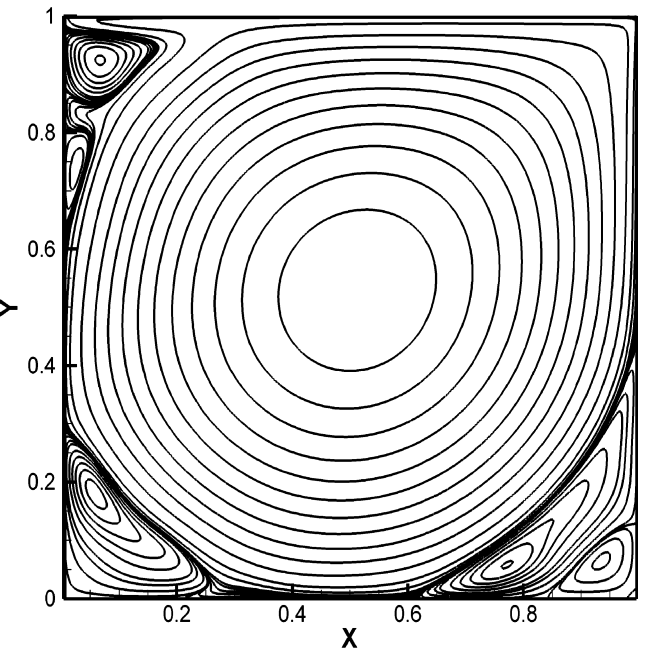

(p) $t=7.5 \mathrm{~s}$

Fig. 9. (continued)

Thus, the study for validating the grid convergence cannot be carried out for $R e=20,000$ any more as no two similar flow states can be found between two sizes of meshes and no reference states can be chosen for comparison.

In Figs. 11 the stream function contours at various times obtained by the 5th-order SUCS scheme for Re $=20,000$ are displayed. The mesh resolution is $64 \times 64$ zone. The initial state denoted by Fig. 11(a) is obtained after a continuous computation time over $6000 \mathrm{~s}$ from the static state is completed. It is seen first from this figure that the flow state is aperiodic because no two similar states can be found. Moreover, a unique vortex motion can be observed evidently: a solitary vortex (denoted by the red arrow) is shedding from the corner of the cavity and moving around the cavity along the boundary.

In order to further validate the non-periodic nature for the above case, the computation is run continuously and the results after $2000 \mathrm{~s}$ are shown in Figs. 12 and 13. First, we obtain a different result, as shown in Figs. 12 in which the flow state is still non-periodic but a solitary vortex (denoted by the red arrow) is moving around the cavity along the inner closed streamlines not along the boundary. Then, in Figs. 13 a solitary vortex can be observed again moving around the cavity along the boundary. The process shown in Figs. 13 is somewhat similar to but not the same as that shown in Figs. 11 for the vortex structures at the corners are obviously different.

As the last instance, we simulate the flows in the lid-driven cavity at $R e=20,000$ by using the 5th-order SUCS scheme and a uniform grid of size $(128 \times 128)$. The stream function contours are presented in Figs. 14 and 15 . We remark here that the initial state portrayed by Fig. 14(a) is obtained after a computation time over $4000 \mathrm{~s}$ from the static state is completed. As previously, we cannot find two similar flow states at different moments in these results even such a sufficiently long computation time has been spent. This demonstrate the non-periodic nature of the flow at $R e=20,000$. Also, the flow 


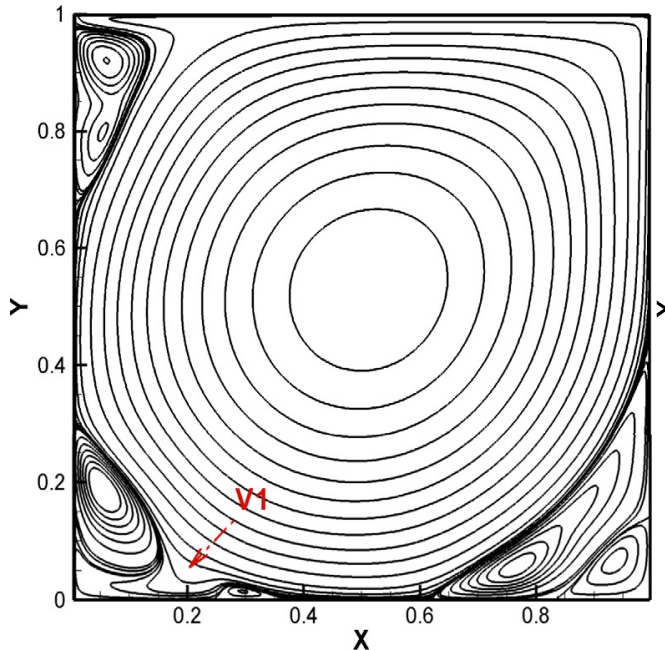

(a) $t=8 \mathrm{~s}$

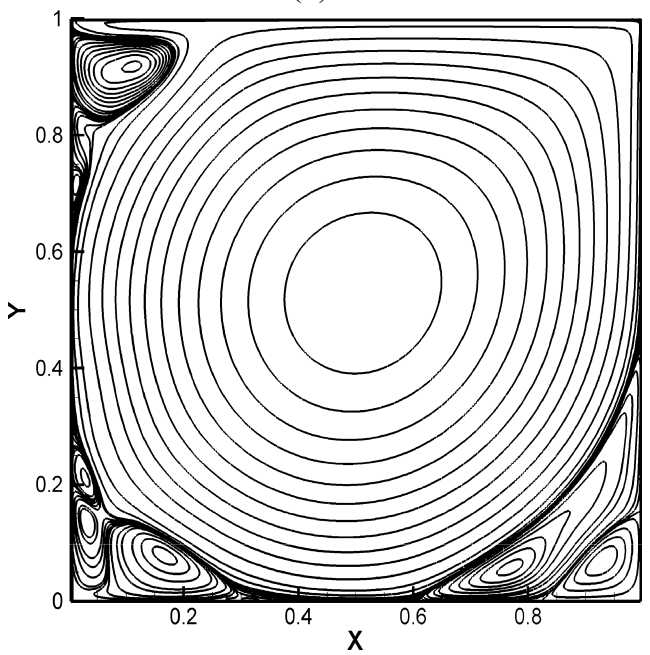

(c) $t=9 \mathrm{~s}$

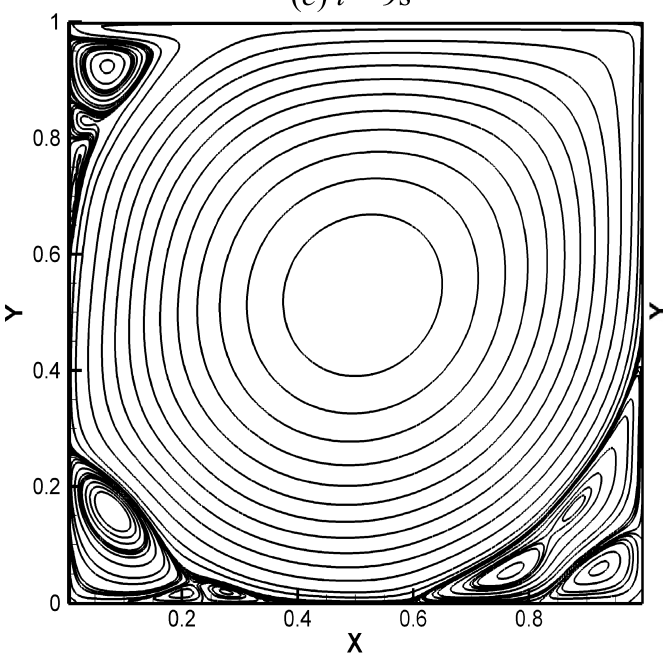

(e) $t=9.75 \mathrm{~s}$

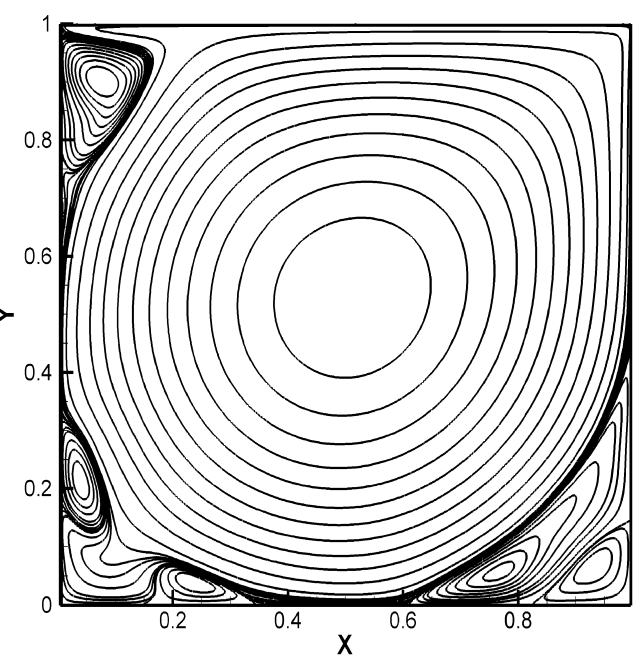

(b) $t=8.5 \mathrm{~s}$

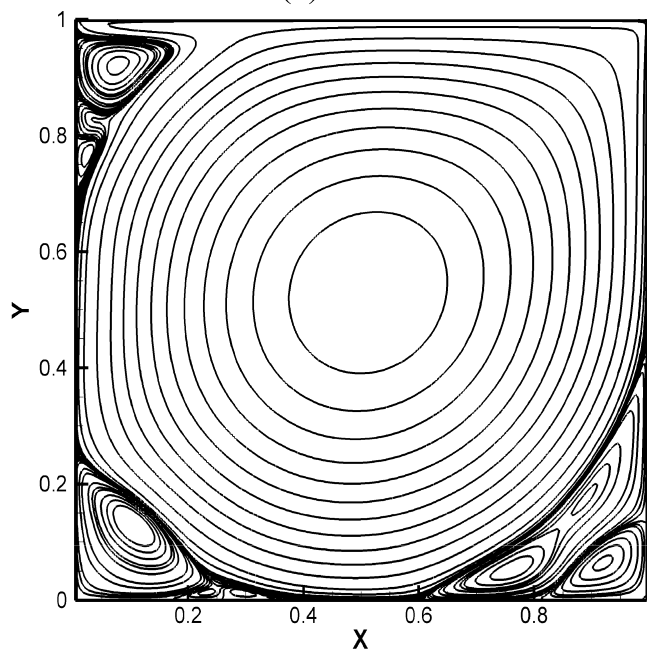

(d) $t=9.5 \mathrm{~s}$

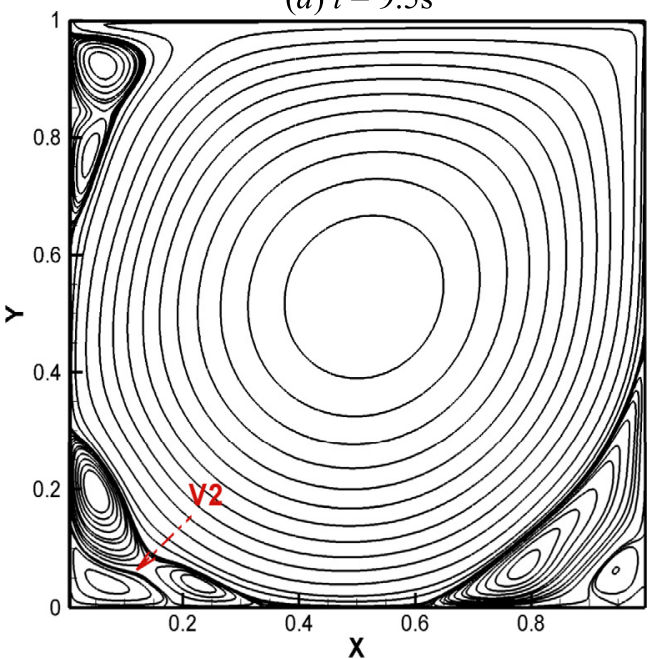

(f) $t=10.25 \mathrm{~s}$

Fig. 10. A consecutive cycle of the stream function contours of the flow in a lid-driven cavity for $R e=10,000$ solved using the 5 th-order SUCS scheme and a uniform grid of size $(256 \times 256)$. The periodic feature can be evidenced from comparing the similarities between Fig. $9(0)$ and Fig. $10(v)$ and between Fig. 9(p) and Fig. 10(w). 

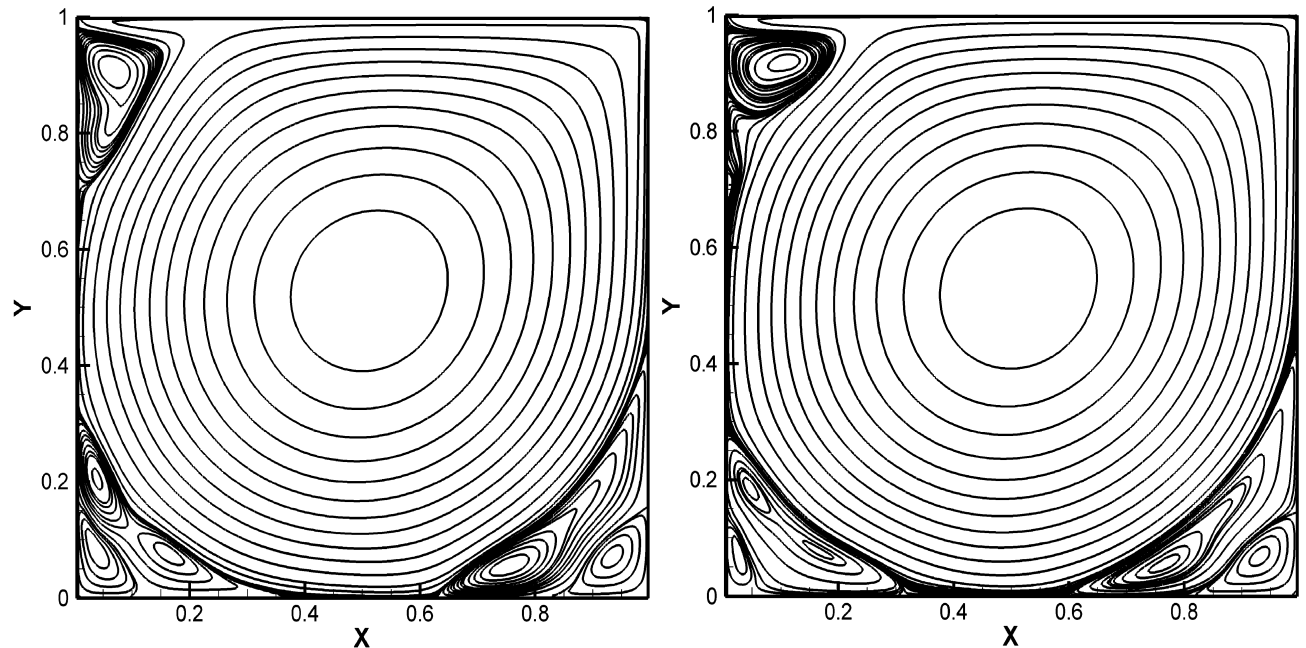

(g) $t=10.75 \mathrm{~s}$

(h) $t=11.5 \mathrm{~s}$
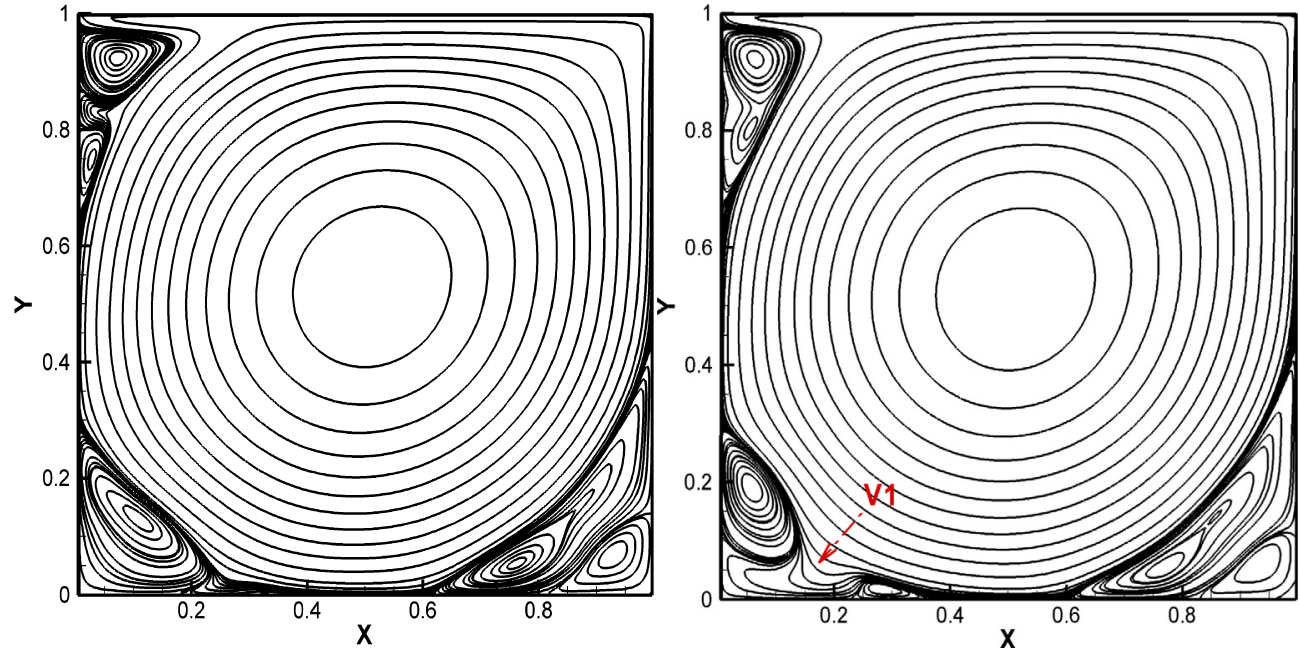

(i) $t=12 \mathrm{~s}$

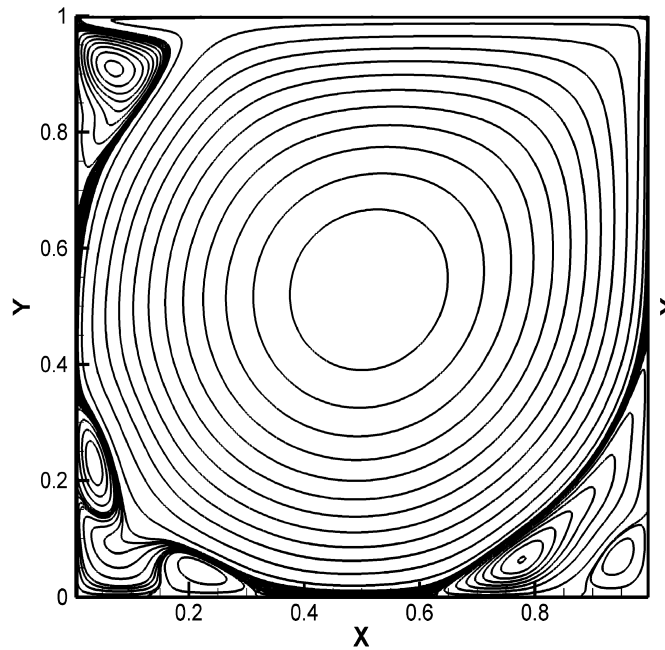

(j) $t=12.5 \mathrm{~s}$

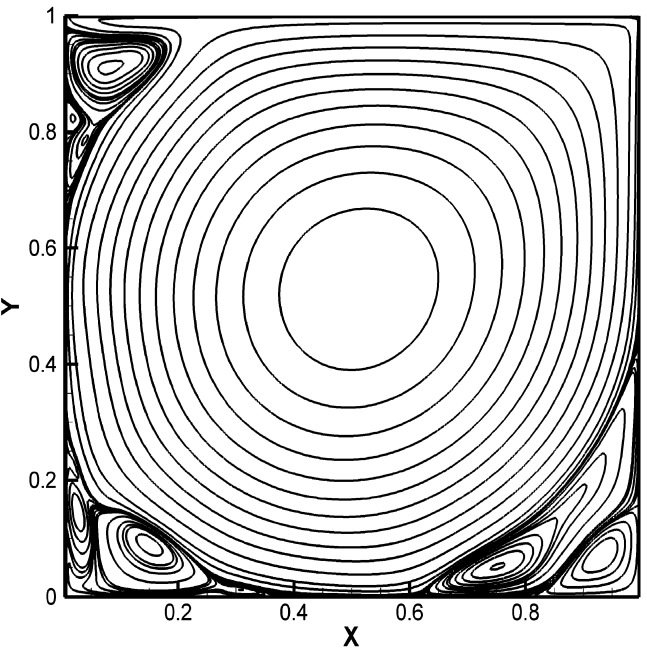

(l) $t=13.5 \mathrm{~s}$

Fig. 10. (continued) 


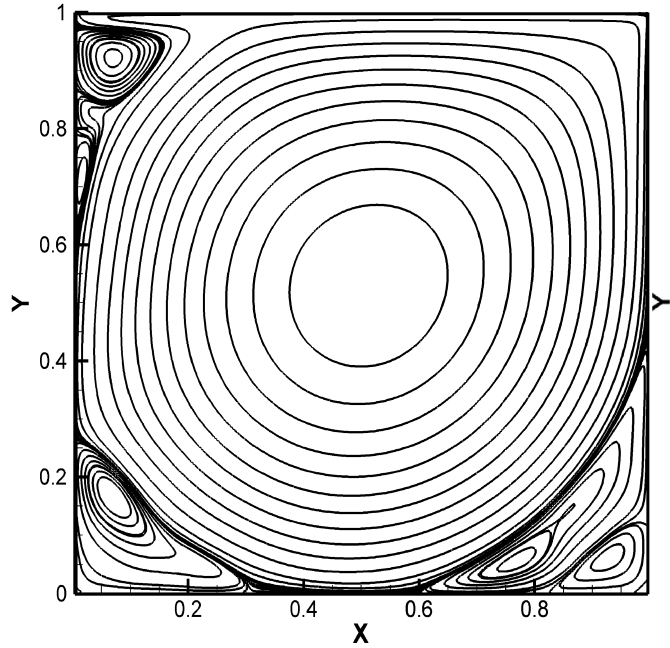

(m) $t=14.25 \mathrm{~s}$

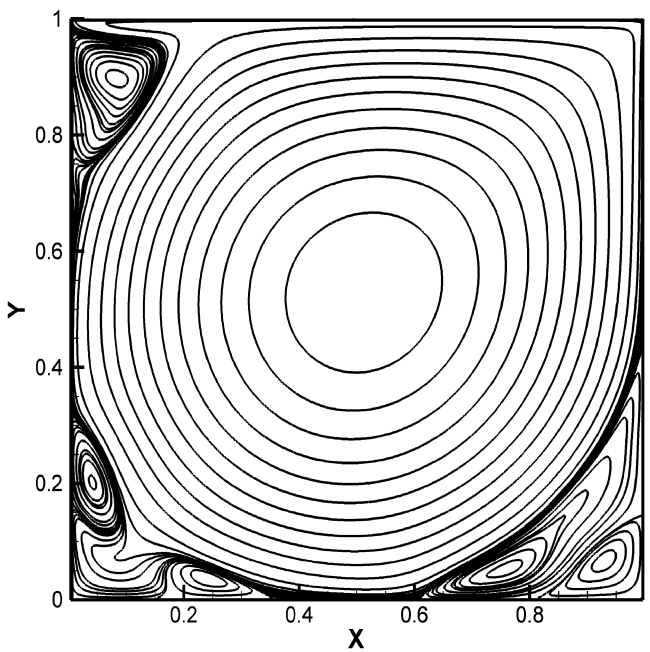

(o) $t=15.5 \mathrm{~s}$

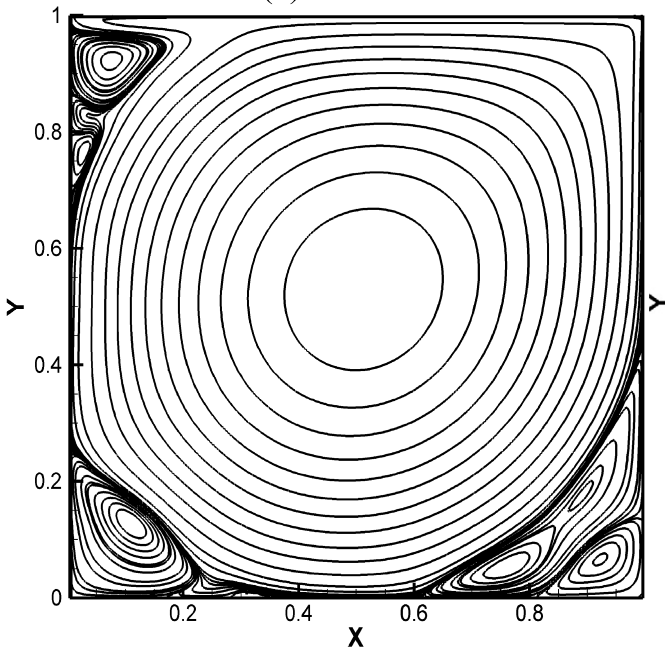

(q) $t=16.5 \mathrm{~s}$

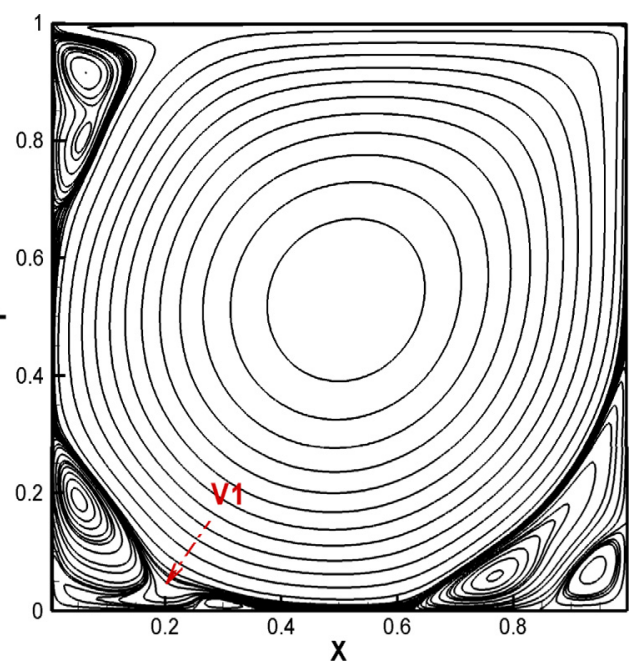

(n) $t=15 \mathrm{~s}$

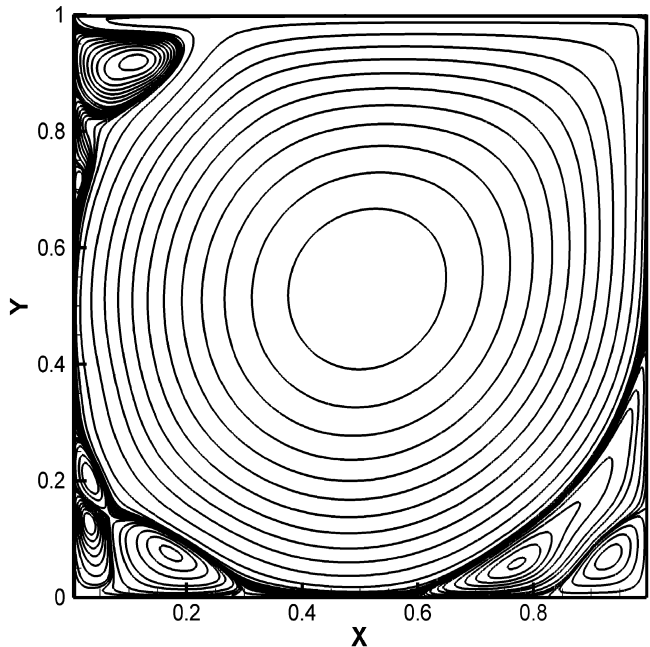

(p) $t=16 \mathrm{~s}$

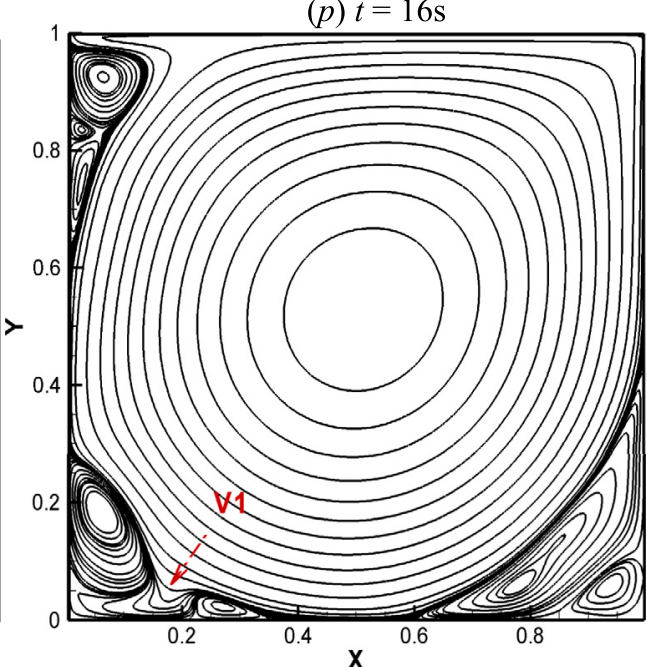

(r) $t=17 \mathrm{~s}$

Fig. 10. (continued) 


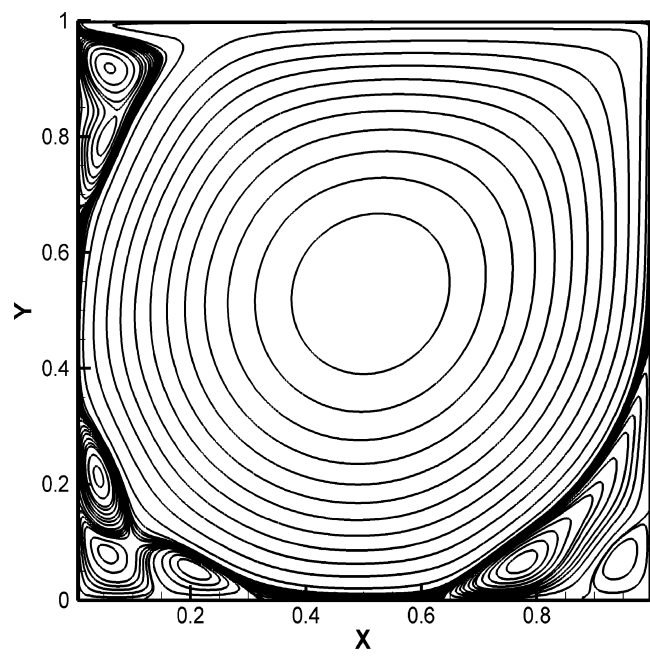

(s) $t=17.5 \mathrm{~s}$

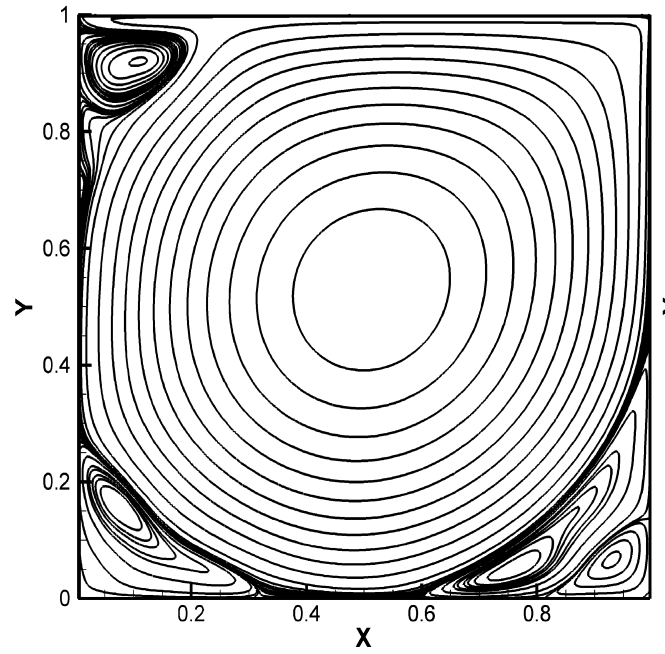

(v) $t=18.5 \mathrm{~s}$

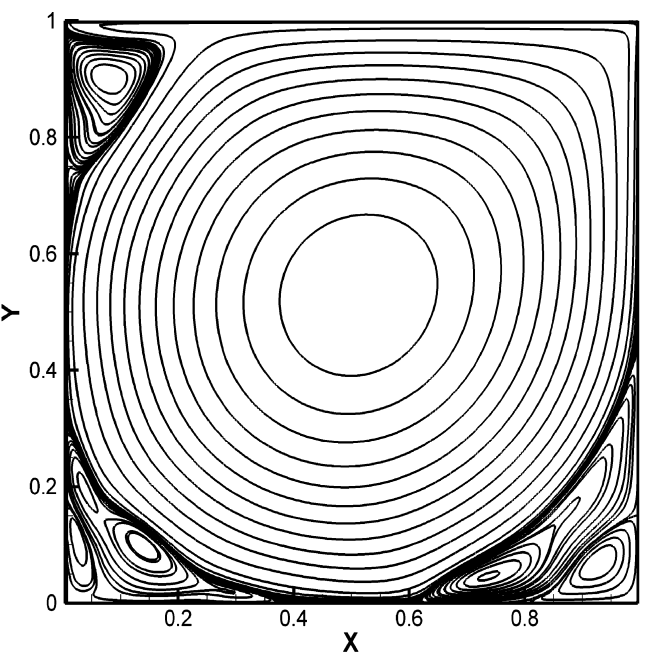

(u) $t=18 \mathrm{~s}$

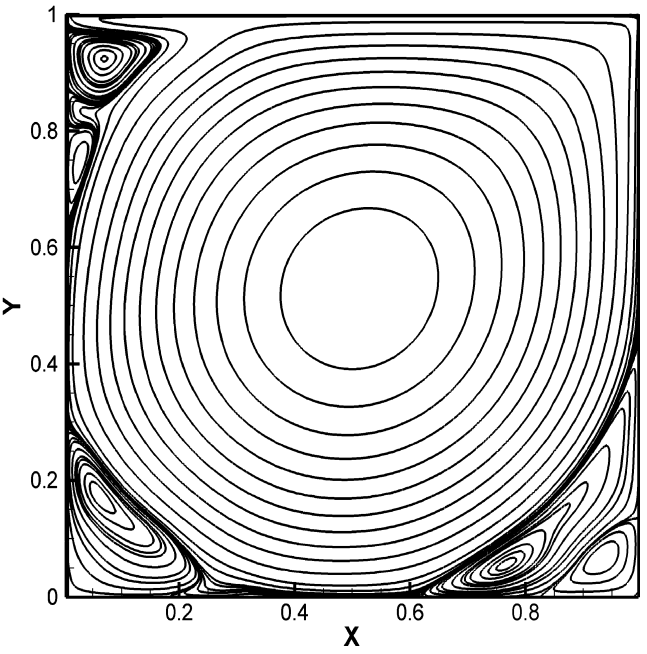

(w) $t=19 \mathrm{~s}$

Fig. 10. (continued)

details are quite complex than the case with $R e=10,000$, mostly in that more sub-processes of the vortex are generated under this smaller Reynolds number.

The computation times shown in Figs. 14 and 15 are totally rather long. So, it is not necessary to present all of the results in the paper but select some representative ones to exhibit that at the local region (top-left corner) of the cavity repeated flow states can still exit. This conclusion comes from the comparison between the areas enclosed by the red dashed lines and those by the green dash-dotted lines at the top-left corner of the cavity where two types of vortex structures are generated repeatedly. Note that the time period demarcated by a same vortex structure can still be different. For example, the time period between Figs. 14(a) and (f) is easily found to be $2 \mathrm{~s}$ and that between Figs. 14(g) and (j) is $1.75 \mathrm{~s}$. Moreover, from comparing Figs. 14(i) and ( $\mathrm{l}$ ) and comparing Figs. 14(m) and ( $\mathrm{r}$ ) the time periods for these two processes can be approximated by $(16-11.75) / 2=2.13 \mathrm{~s}$ and $(31-27.5) / 2=1.75 \mathrm{~s}$, respectively.

Although it has been remarked previously that the grid convergence cannot be validated effectively from the computations with $R e=20,000$, we can still find some evidences to show that the flow states described by a finer grid comprise some features of the flows described by the coarser grids. In Figs. 15 a solitary vortex (denoted by the red arrow) can be observed shedding from the bottom-right corner and moving along the boundary to the bottom-left corner of the cavity. This process is somewhat analogous to that shown in Figs. 11 and 13.

In the end, with the purpose of manifesting the high resolution property of SUCS scheme, we compare the results for $R e=20,000$ between the SUCS and explicit upwind schemes. Figs. 16 show the stream function contours at time $t=$ $26 \mathrm{~s}$ computed by the 3rd- and 5th-order explicit upwind schemes and 3rd- and 5th-order SUCS schemes. The differences between the results obtained by these schemes are obvious. Compared to the explicit scheme, more small vortices are 


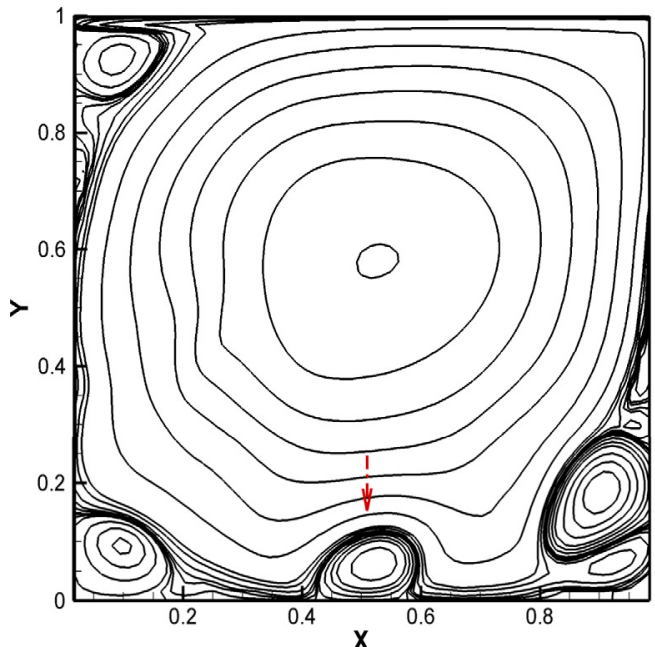

(a) $t=0 \mathrm{~s}$

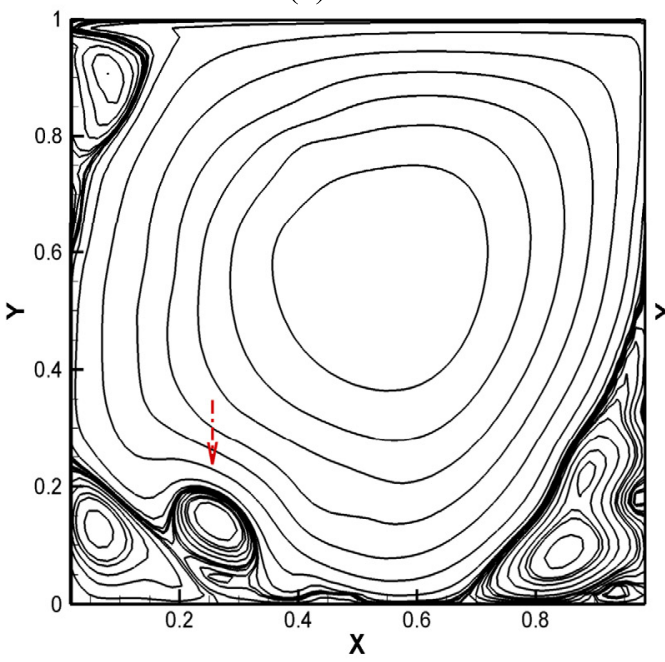

(c) $t=2 \mathrm{~s}$

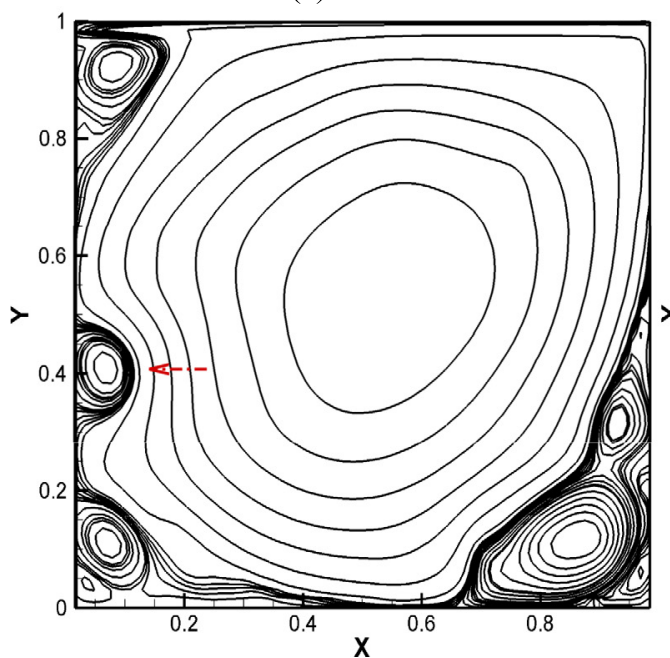

(e) $t=4 \mathrm{~s}$

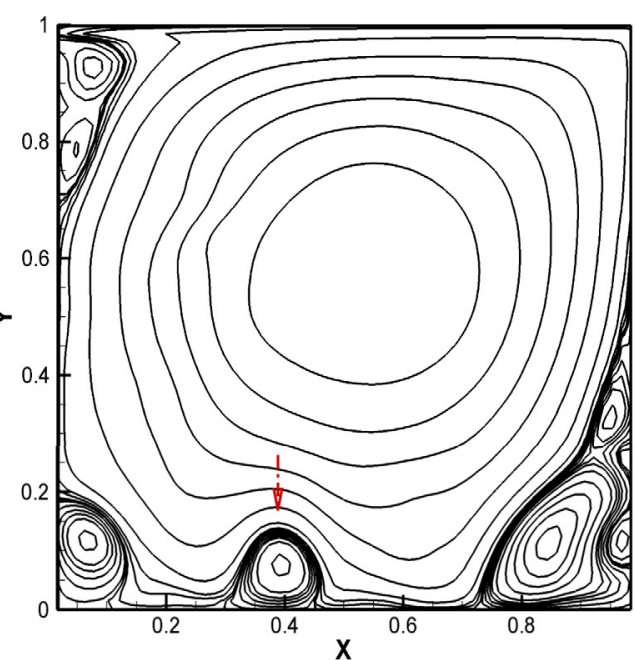

(b) $t=1 \mathrm{~s}$

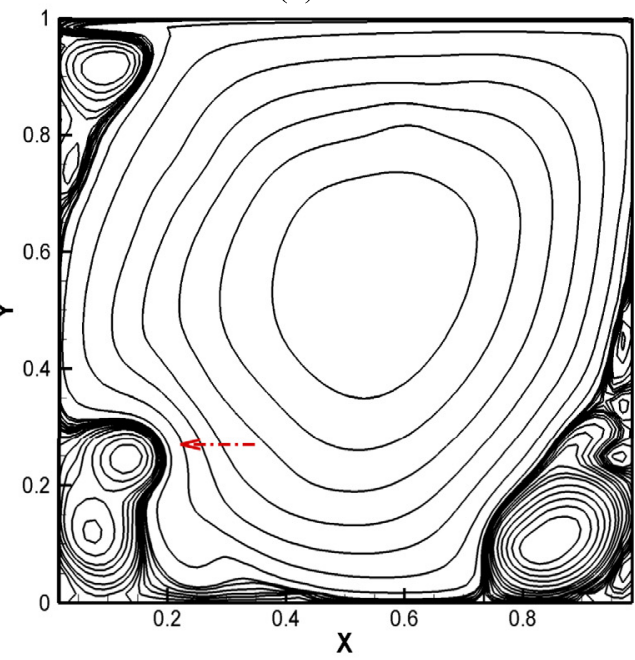

(d) $t=3 \mathrm{~s}$

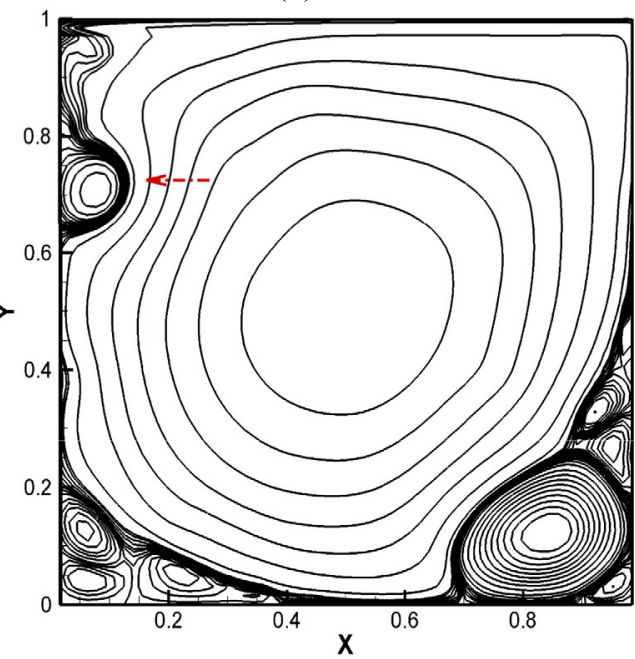

(f) $t=6 \mathrm{~s}$

Fig. 11. The stream function contours of the flow in a lid-driven cavity for $R e=20,000$ solved using the 5 th-order SUCS scheme and a uniform grid of size $(64 \times 64)$. The initial state depicted in Fig. 11(a) is obtained after a continuous computation time over $6000 \mathrm{~s}$ from the static state is completed. A solitary vortex (denoted by the red arrow) can be observed evidently shedding from the corner of the cavity and moving around the boundary. 


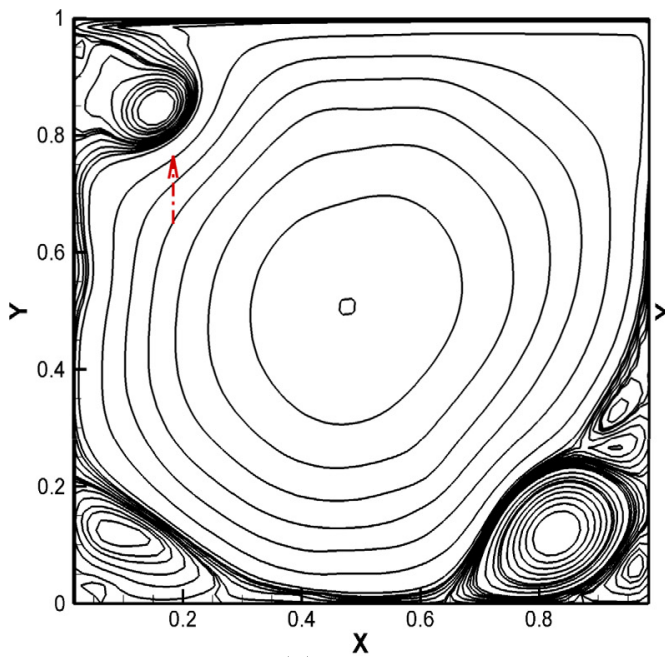

(g) $t \stackrel{\mathrm{X}}{=} 7 \mathrm{~s}$

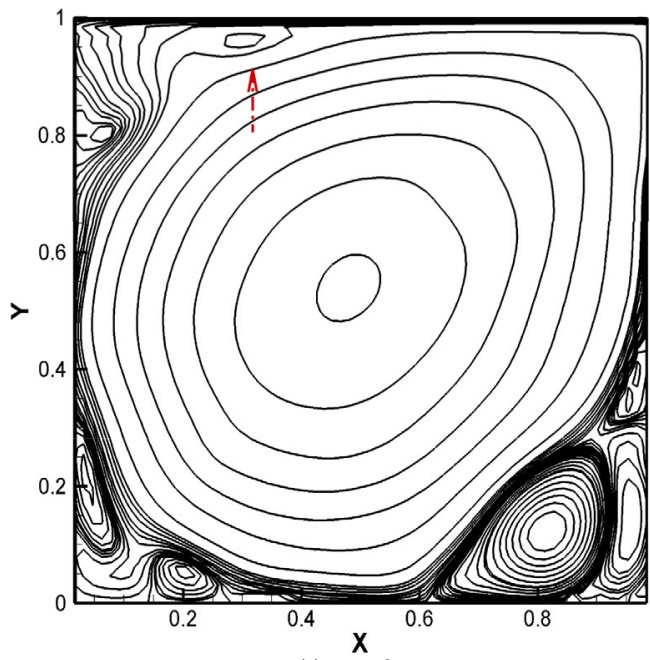

(i) $t=9 \mathrm{~s}$

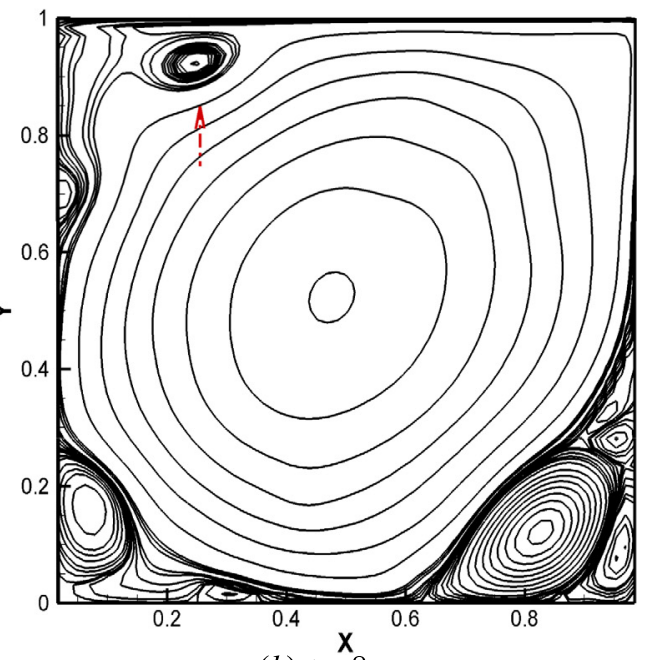

(h) $t=8 \mathrm{~s}$

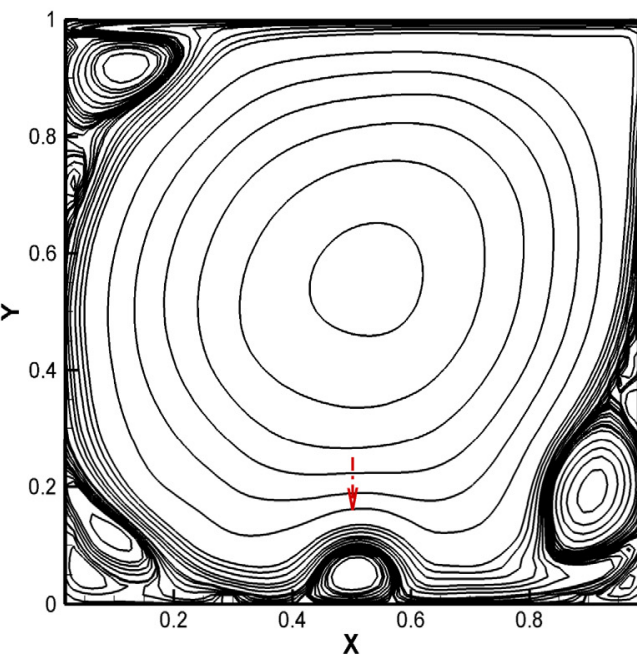

(j) $t=14 \mathrm{~s}$

Fig. 11. (continued)

generated at the bottom of the cavity upon using the 5th-order SUCS scheme, which are clearly illustrated in the area enclosed by the red dashed lines.

\section{Derivations of the generalized forms of SUCS schemes}

In this section, we attempt to derive the generalized forms of the SUCS schemes for non-uniform grids. As mentioned in the introduction, derivations of the compact schemes for non-uniform grids have been attempted previously by using the Hermite interpolation polynomial based methods $[8,9,17]$. However, from this kind of methodology the truncation errors (TE) of the difference schemes cannot be obtained simultaneously and, as a consequence, the basic numerical features of the schemes (including order of accuracy and dissipation or dispersion property) cannot be elucidated directly. In this paper we apply a new stepwise weighted summation method together with the mathematical induction principle to derive the generalized form for the compact schemes. With this method, not only the fully explicit presentations of the coefficients but also the TE of the schemes can be obtained simultaneously.

It should be mentioned particularly that during the revision of this paper the author noticed a new reference [31] in which a new compact scheme for the non-uniform grids was derived by using a similar weighted summation method. Some of the results in this literature are similar to those in section 5.1 .1 of this paper. Therefore, it seems necessary to point out here that an earlier edition of this paper was submitted to the same journal on 16 March 2015 (the manuscript number was JCOMP-D-15-00335 and one critical review suggested adding the GSA as presented in section 3 of the current paper) in which the derivations of the compact schemes for non-uniform grids are completely the same as those in the current paper. The above statement can be regarded as a clarification for the originality of this paper. 


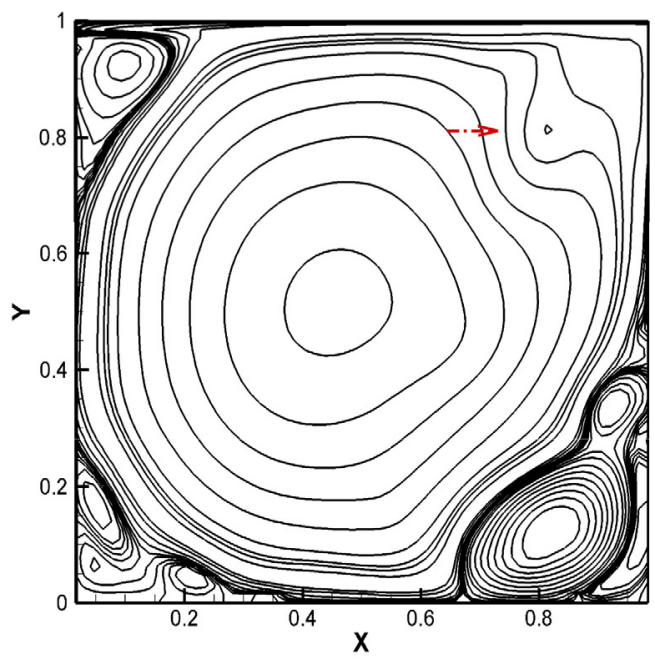

(a) $t=2014 \mathrm{~s}$

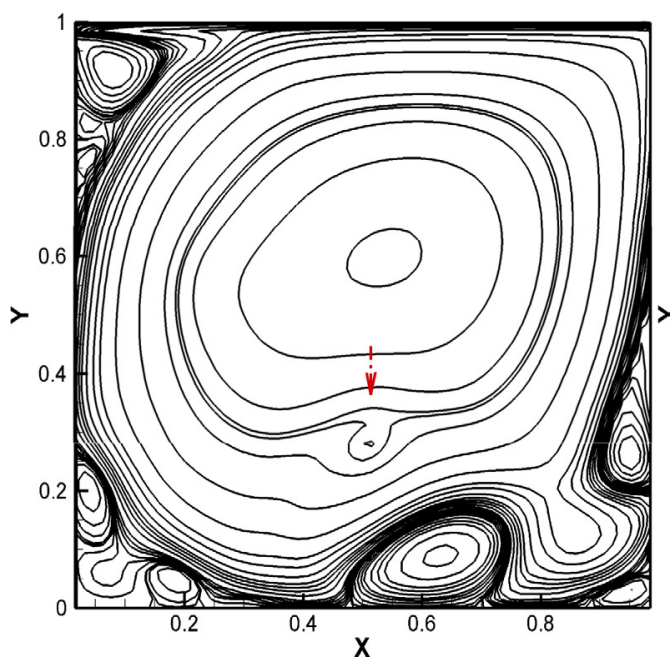

(c) $t=2019 \mathrm{~s}$

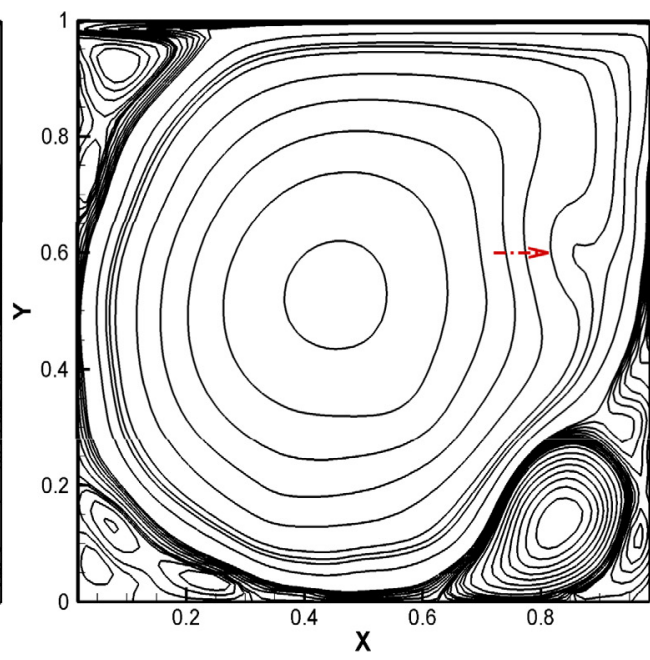

(b) $t=2015 \mathrm{~s}$

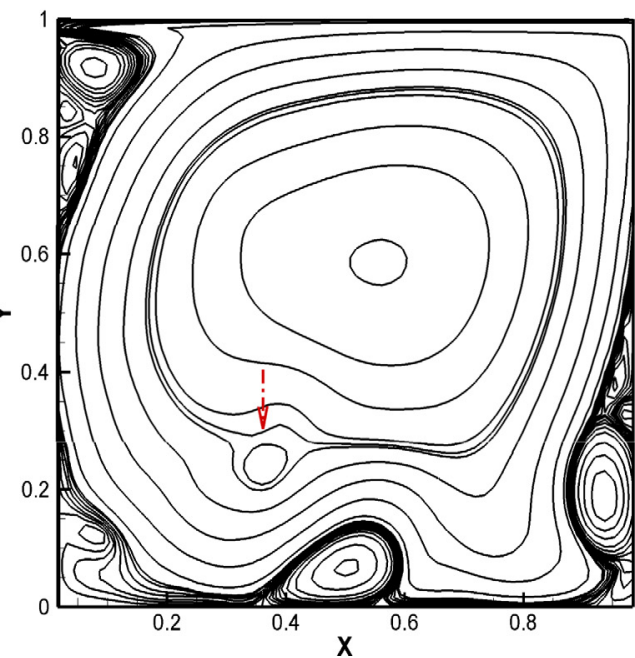

(d) $t=2020 \mathrm{~s}$

Fig. 12. The stream function contours of the flow in a lid-driven cavity for $R e=20,000$ solved using the 5 th-order SUCS scheme and a uniform grid of size $(64 \times 64)$. A solitary vortex (denoted by the red arrow) is exhibited moving around the cavity along inner closed streamlines.

\subsection{Bi-diagonal}

\subsubsection{3rd-order of accuracy $\left(B_{3}\right)$}

(i) $\boldsymbol{B}_{\mathbf{2 a}}$. We start our derivations with the bi-diagonal cases. Considering a 2nd-order approximation to $u_{0}^{\prime}, \boldsymbol{B}_{\mathbf{2 a}}$, based on stencil $\left\{\boldsymbol{u}_{-1}^{\prime}, \boldsymbol{u}_{-1}, \boldsymbol{u}_{\mathbf{0}}\right\}$, this problem can be separated into two 1st-order approximations, $B_{1 a}$ and $B_{1 b}$, which are based on stencils $\left\{u_{-1}^{\prime}\right\}$ and $\left\{u_{-1}, u_{0}\right\}$, respectively, and can be expressed as

$$
\begin{aligned}
& B_{1 a}=u_{-1}^{\prime}=u_{0}^{\prime}+\sum_{n=1}^{\infty} e_{1 a}^{(n)} u_{0}^{(n+1)} \\
& B_{1 b}=\frac{u_{-1}-u_{0}}{h_{-1}}=u_{0}^{\prime}+\sum_{n=1}^{\infty} e_{1 b}^{(n)} u_{0}^{(n+1)}
\end{aligned}
$$

in which $h_{l}=x_{l}-x_{0}, u_{l}=u\left(x_{l}\right)$ and $u_{l}^{\prime}=u^{\prime}\left(x_{l}\right)(l=0, \pm 1, \pm 2, \ldots)$. Coefficients of TE of the above two approximations are given by

$$
e_{1 a}^{(n)}=\frac{h_{-1}^{n}}{n !} ; \quad e_{1 b}^{(n)}=\frac{h_{-1}^{n}}{(n+1) !}
$$




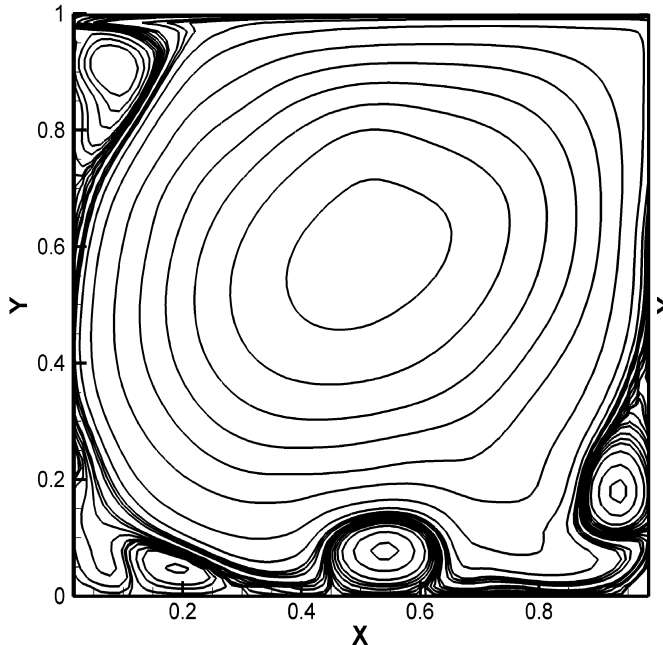

(a) $t=2035 \mathrm{~s}$

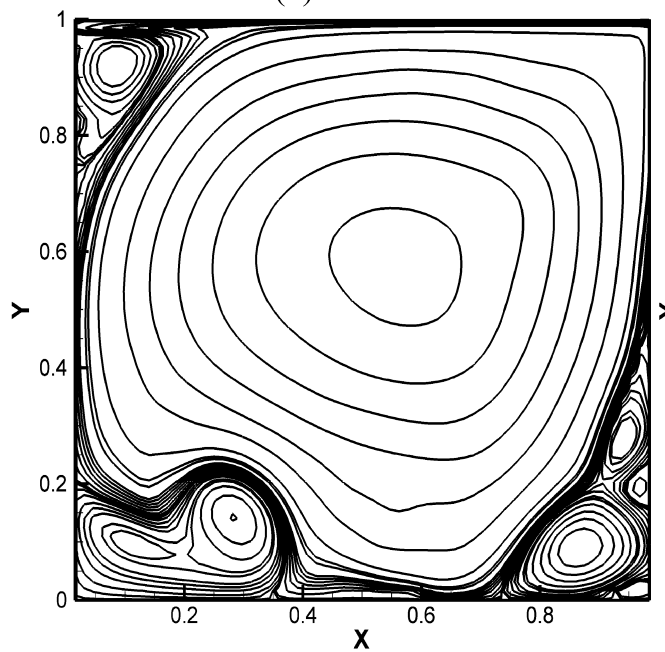

(c) $t=2037 \mathrm{~s}$

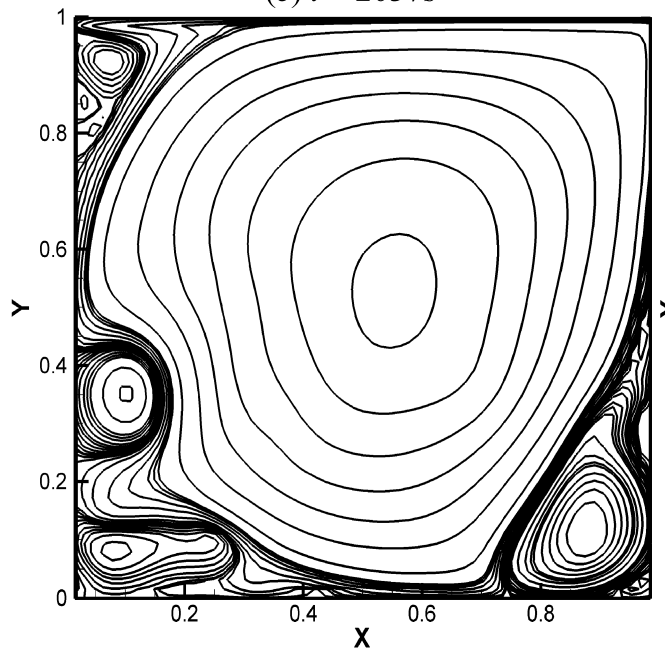

(e) $t=2039 \mathrm{~s}$

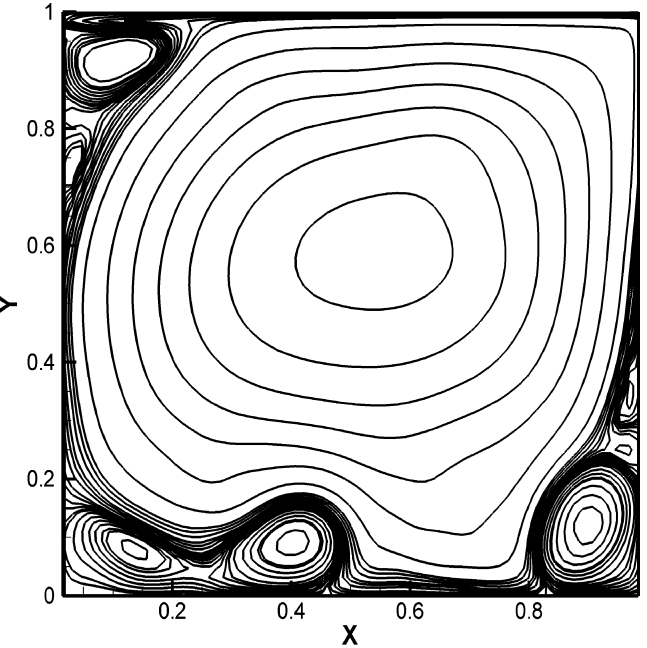

(b) $t=2036 \mathrm{~s}$

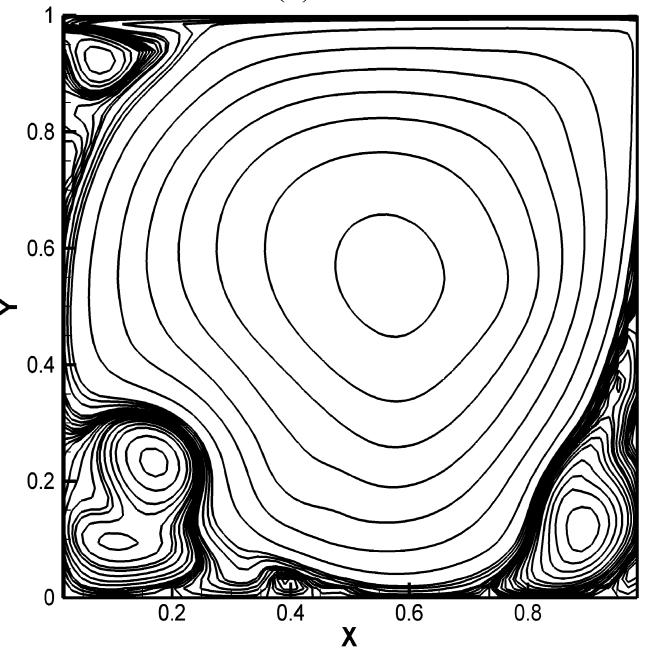

(d) $t=2038 \mathrm{~s}$

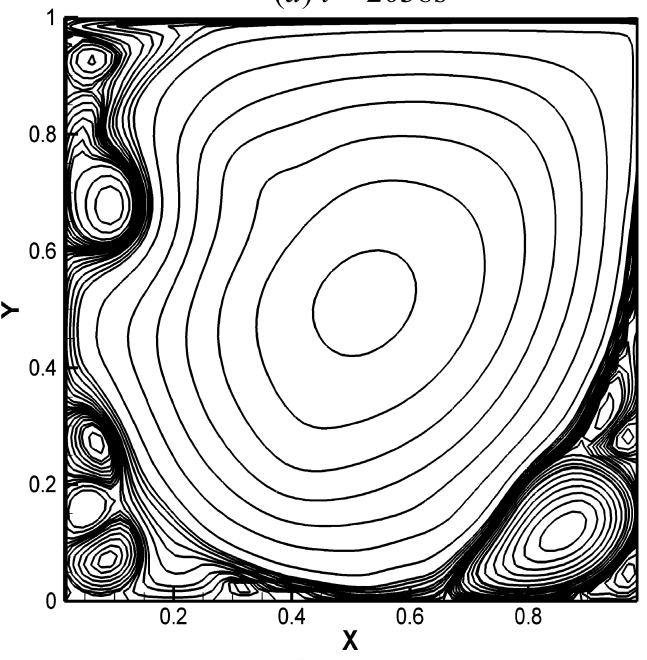

(f) $t=2041 \mathrm{~s}$

Fig. 13. The stream function contours of the flow in a lid-driven cavity for $R e=20,000$ solved using the 5th-order SUCS scheme and a uniform grid of size $(64 \times 64)$. 


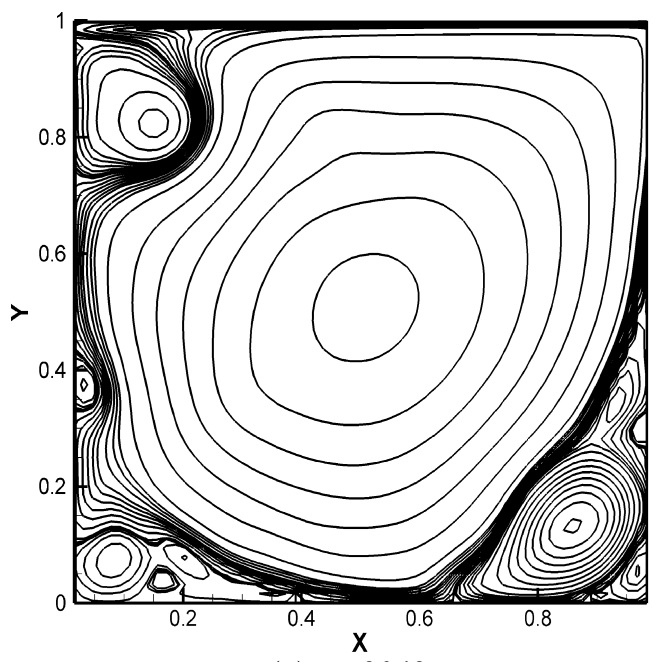

(g) $t=2042 \mathrm{~s}$

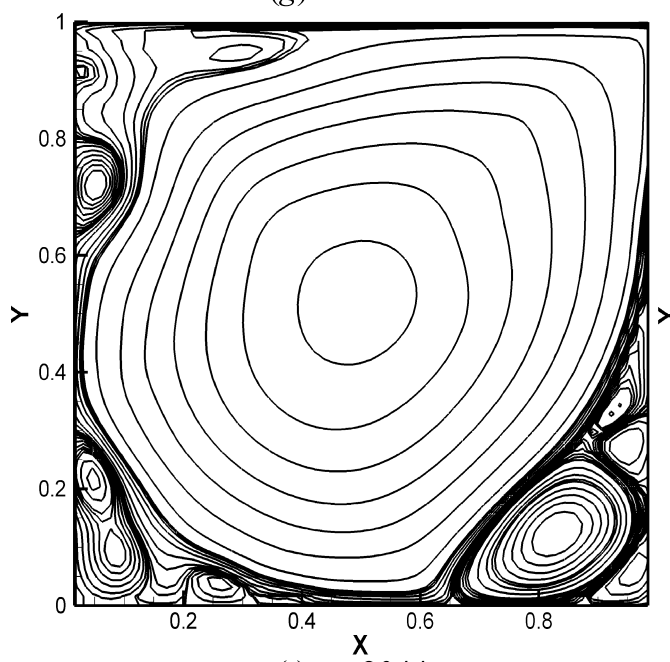

(i) $t=2044 \mathrm{~s}$

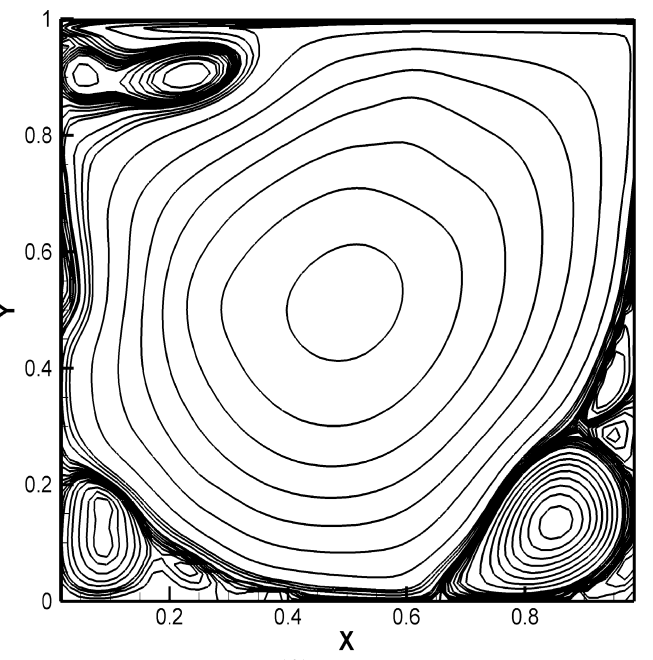

(h) $t=2043 \mathrm{~s}$

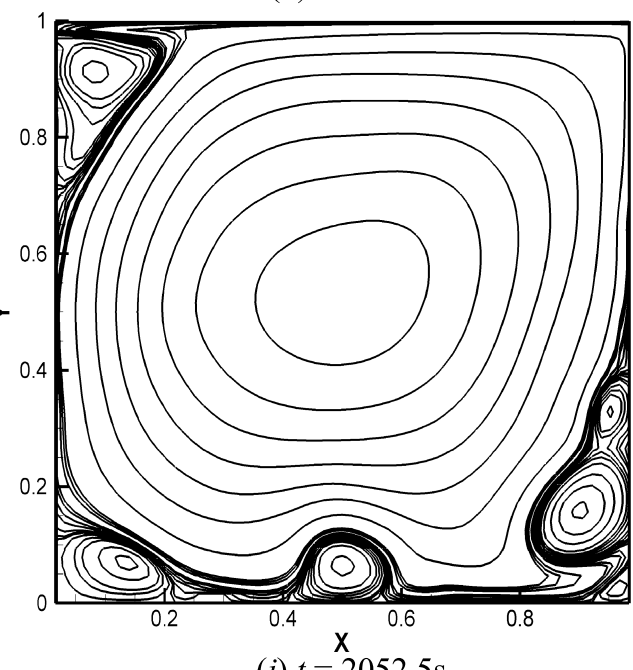

(j) $t=2052.5 \mathrm{~s}$

Fig. 13. (continued)

It is not difficult to realize that a 2nd-order approximation to $u_{0}^{\prime}$ can be yielded from the following weighted summation operation, given by

$$
B_{2 a}=\omega B_{1 a}+(1-\omega) B_{1 b}=u_{0}^{\prime}+\sum_{n=2}^{\infty} e_{2 a}^{(n)} u_{0}^{(n+1)}
$$

in which the weight can be calculated from

$$
\omega=\frac{e_{1 b}^{(1)}}{e_{1 b}^{(1)}-e_{1 a}^{(1)}}=-1
$$

By substituting (26) into (25), the explicit expression for the 2nd-order approximation $B_{2 a}$ can be obtained, given by

$$
B_{2 a}=-u_{-1}^{\prime}+2 \frac{u_{-1}-u_{0}}{h_{-1}}
$$

Also, the TE of $B_{2 a}$ can be obtained from a similar weighted summation procedure

$$
e_{2 a}^{(n)}=\omega e_{1 a}^{(n)}+(1-\omega) e_{1 b}^{(n)}=\frac{(1-n) h_{-1}^{n}}{(n+1) !}
$$




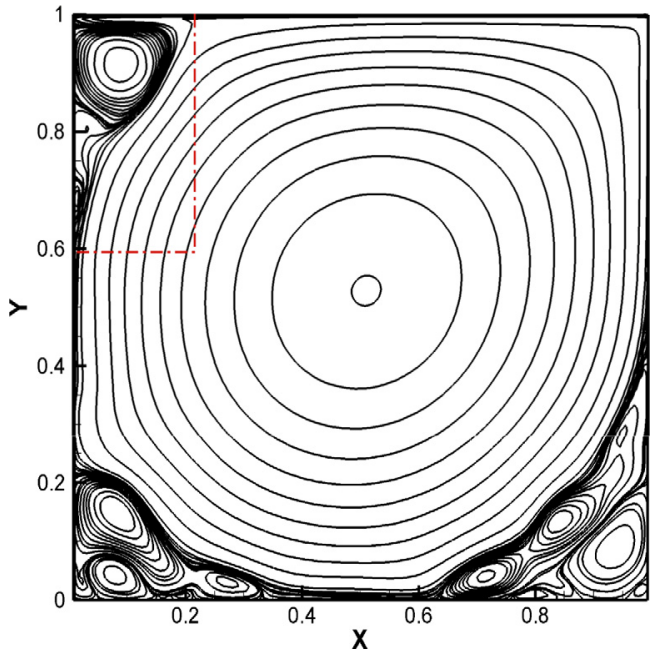

(a) $t=0 \mathrm{~s}$

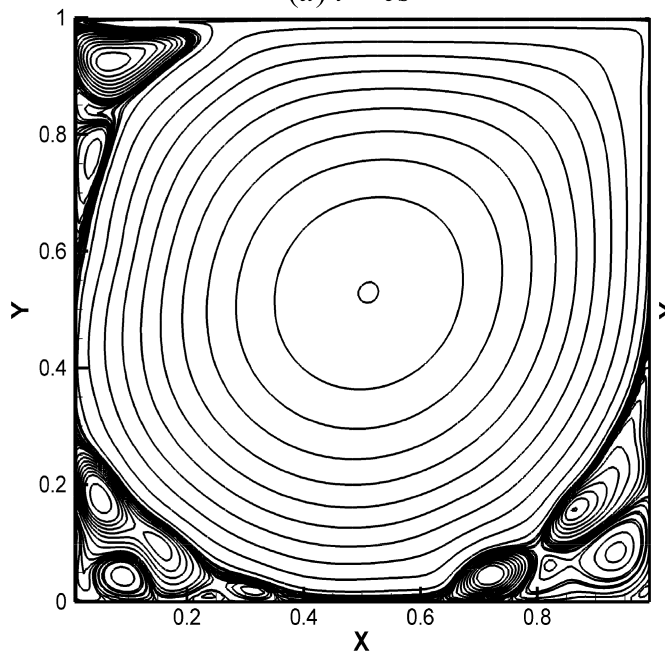

(c) $t=0.75 \mathrm{~s}$

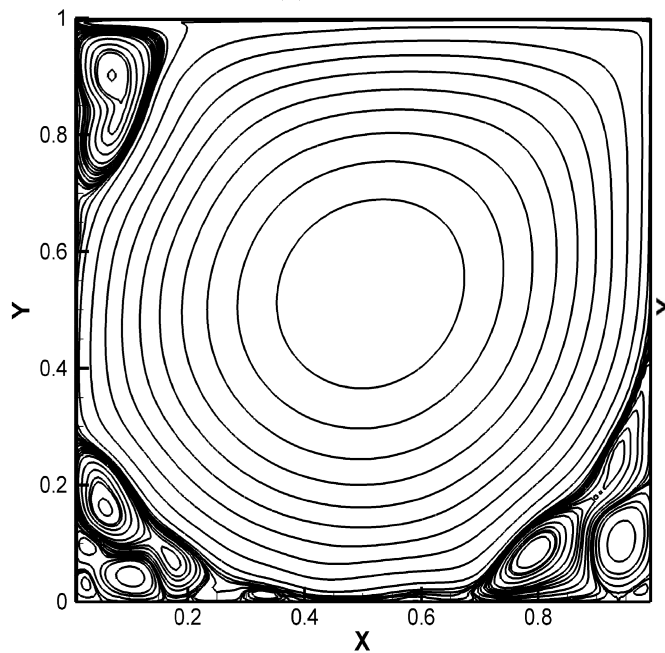

(e) $t=1.5 \mathrm{~s}$

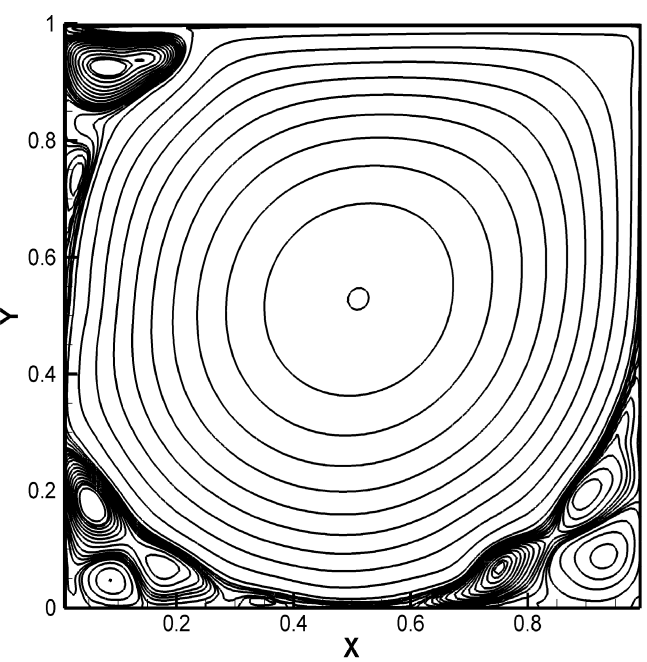

(b) $t=0.5 \mathrm{~s}$

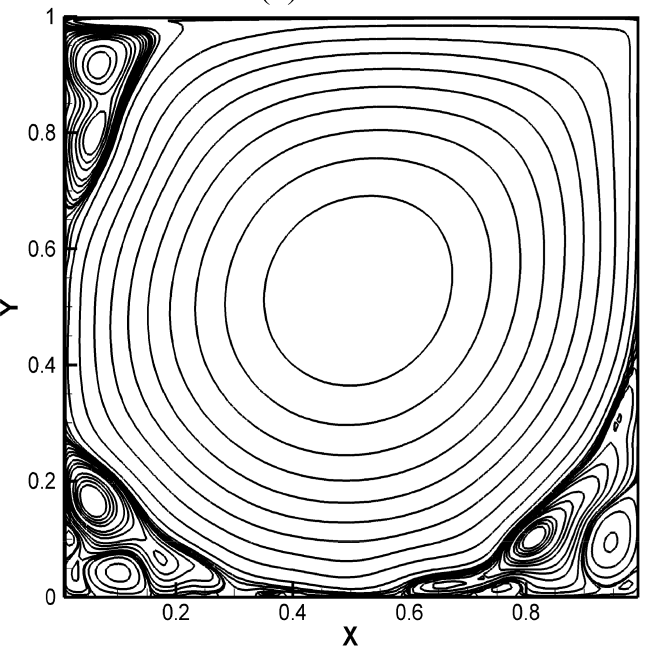

(d) $t=1.25 \mathrm{~s}$

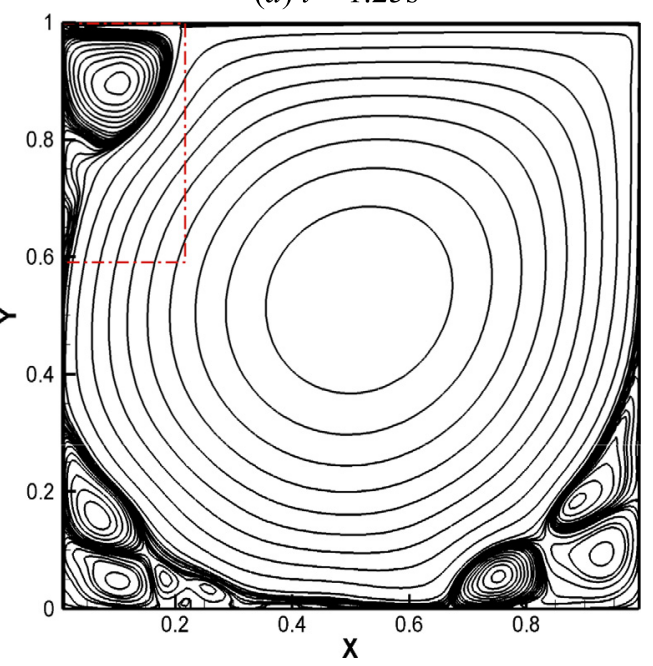

(f) $t=2 \mathrm{~s}$

Fig. 14. Stream function contours for the lid-driven cavity problem at $R e=20,000$, obtained by the 5th-order SUCS scheme using a uniform grid of size $(128 \times 128)$. The initial state portrayed in Fig. $14(\mathrm{a})$ is obtained after a continuous computation time over $4000 \mathrm{~s}$ from the static state is completed. The areas enclosed by red (dashed) lines and green (dash-dotted) lines at the top-left corner of the cavity represent two types of repeatedly generated vortexes. 


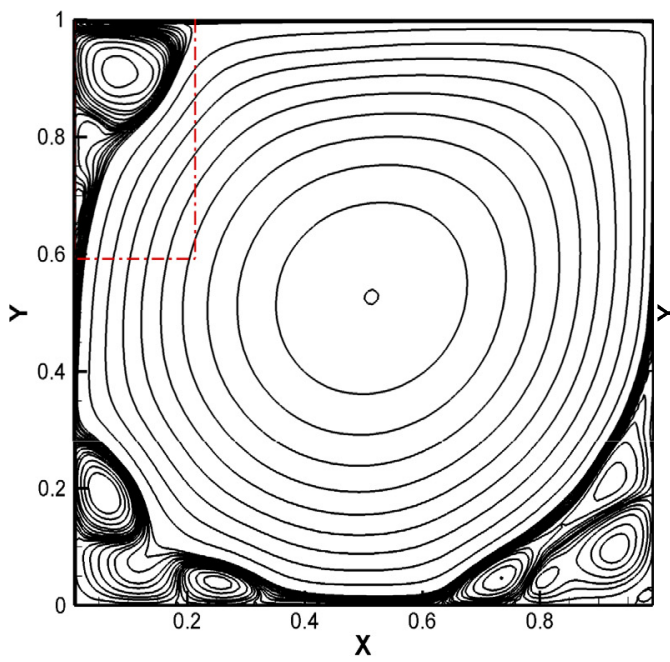

(g) $t=10.75 \mathrm{~s}$

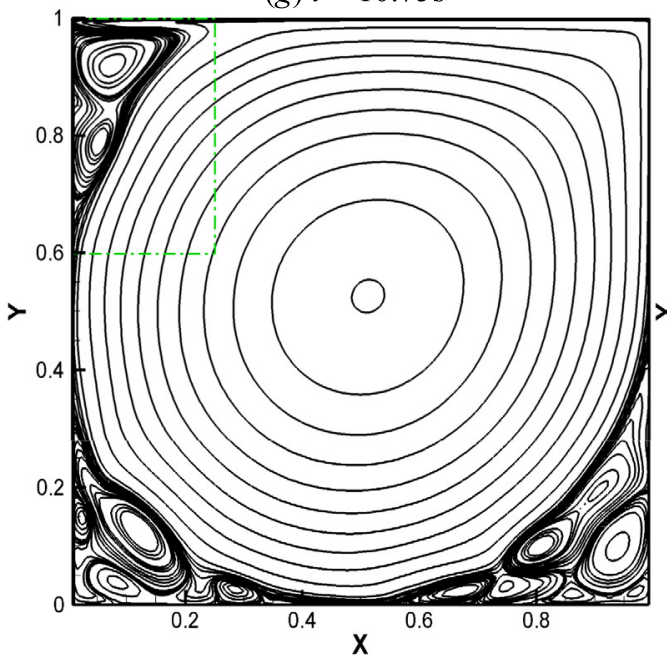

(i) $t=11.75 \mathrm{~s}$

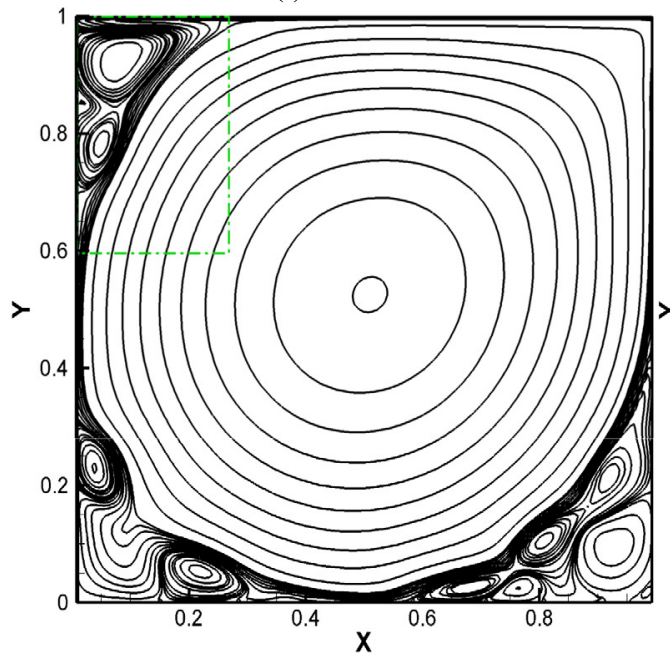

(k) $t=13.5 \mathrm{~s}$

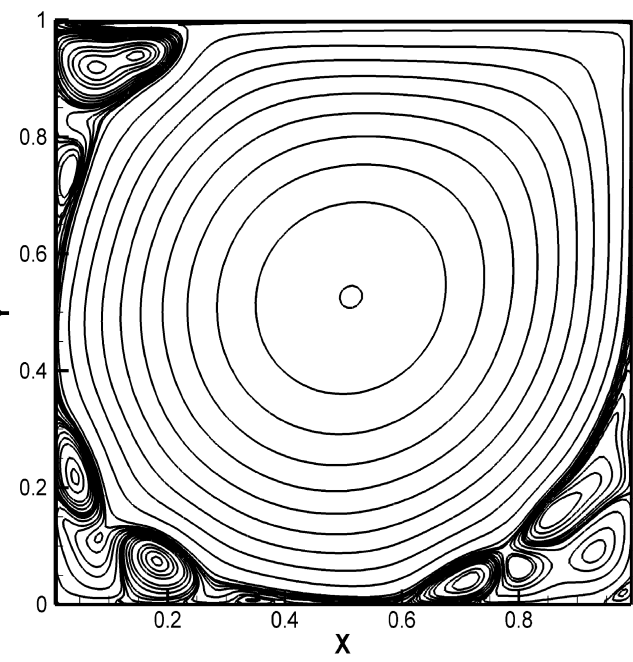

(h) $t=11.25 \mathrm{~s}$

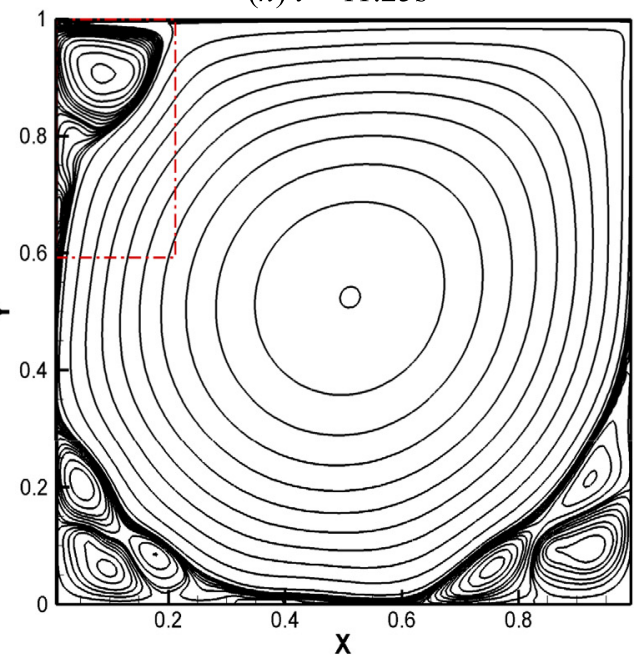

(j) $t=12.5 \mathrm{~s}$

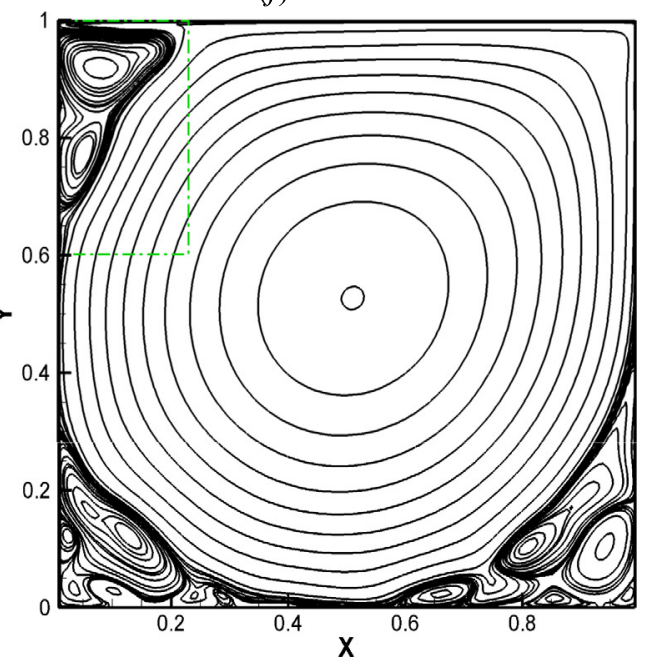

(l) $t=16 \mathrm{~s}$

Fig. 14. (continued) 


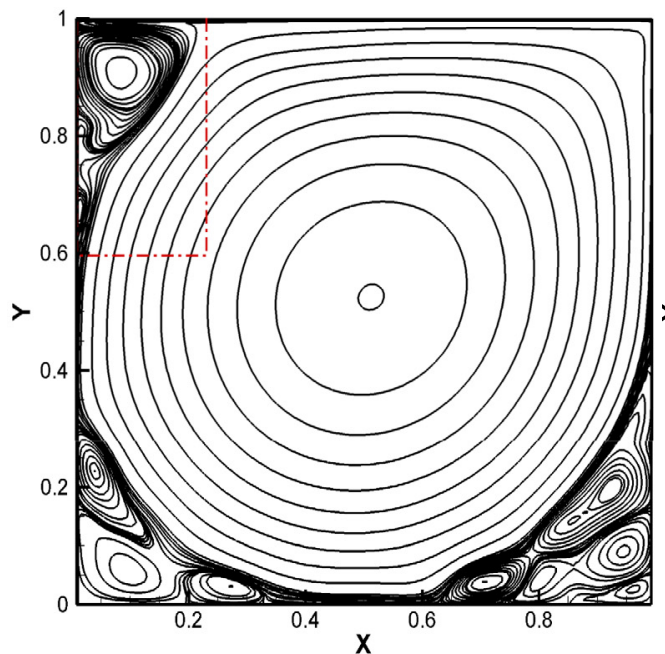

(m) $t=27.5 \mathrm{~s}$

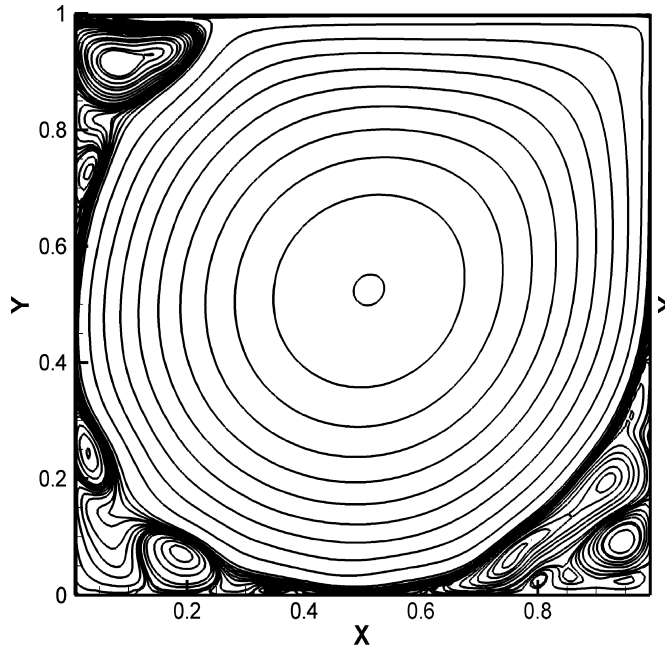

(o) $t=28 \mathrm{~s}$

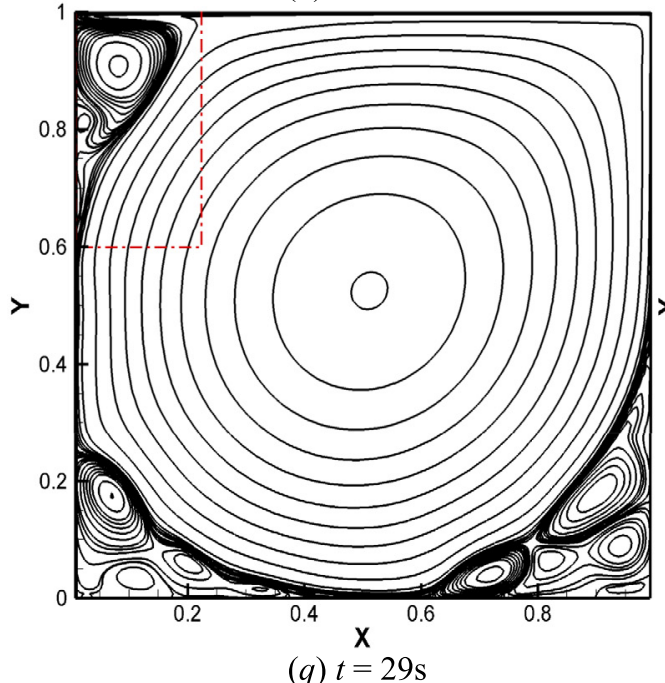

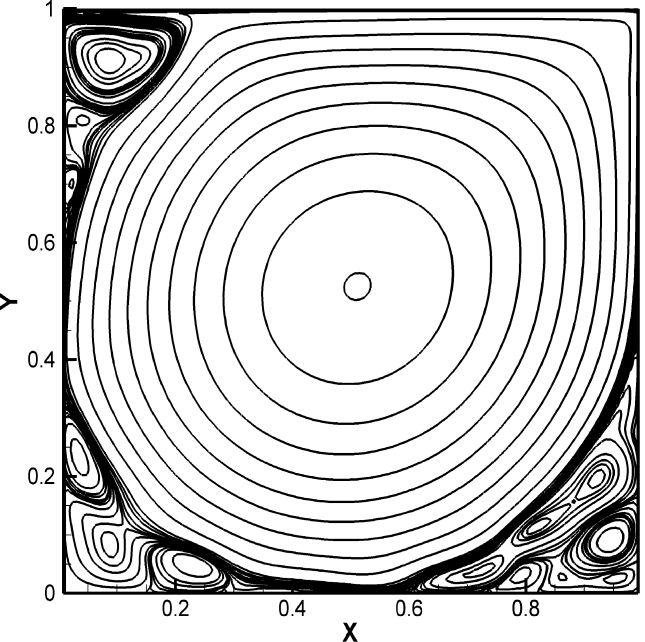

(n) $t=27.75 \mathrm{~s}$

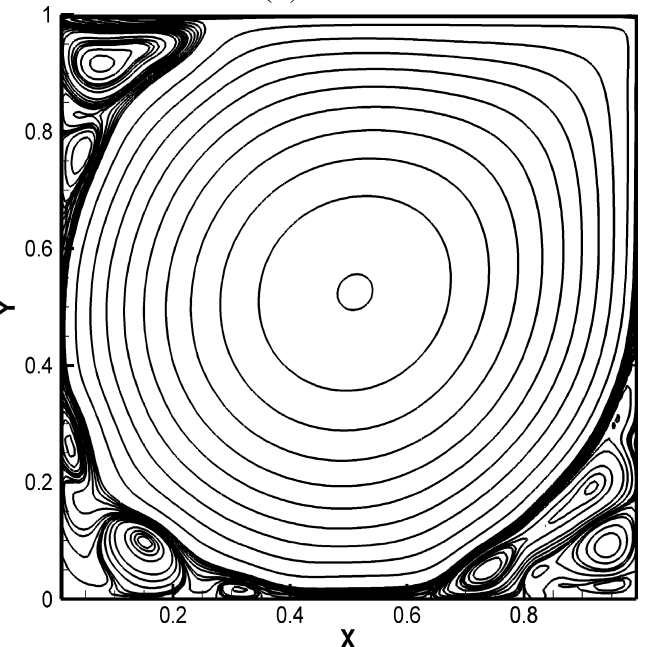

(p) $t=28.25 \mathrm{~s}$

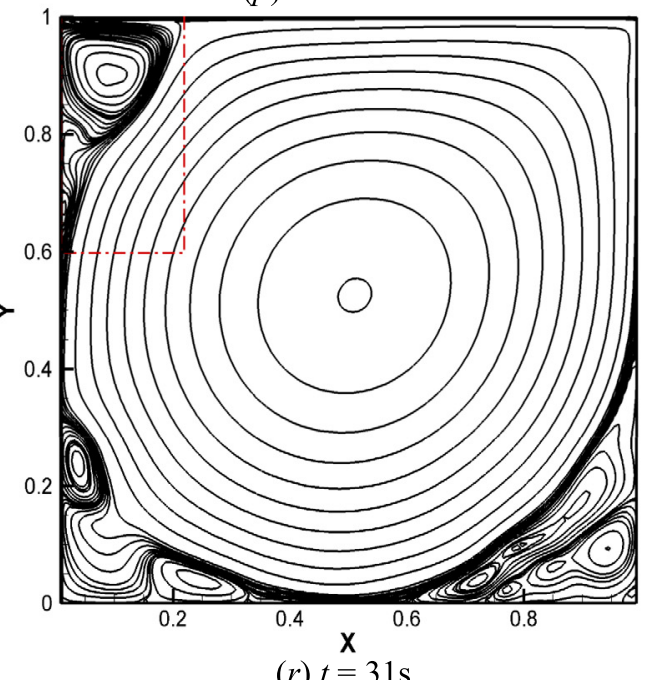

(r) $t=31 \mathrm{~s}$

Fig. 14. (continued) 


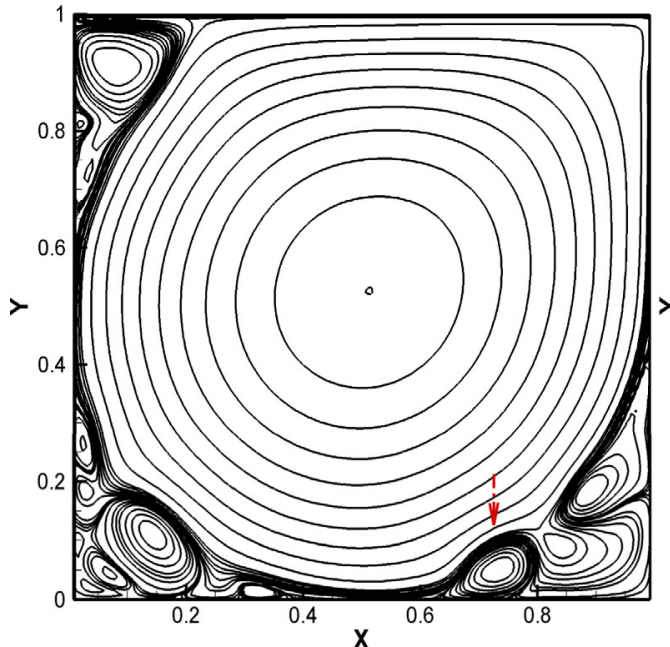

(a) $t=20.25 \mathrm{~s}$

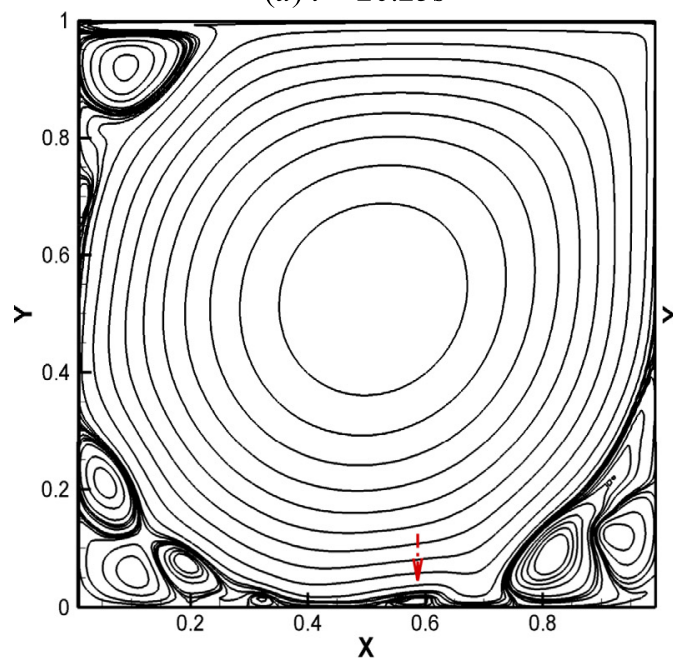

(c) $t=21.25 \mathrm{~s}$

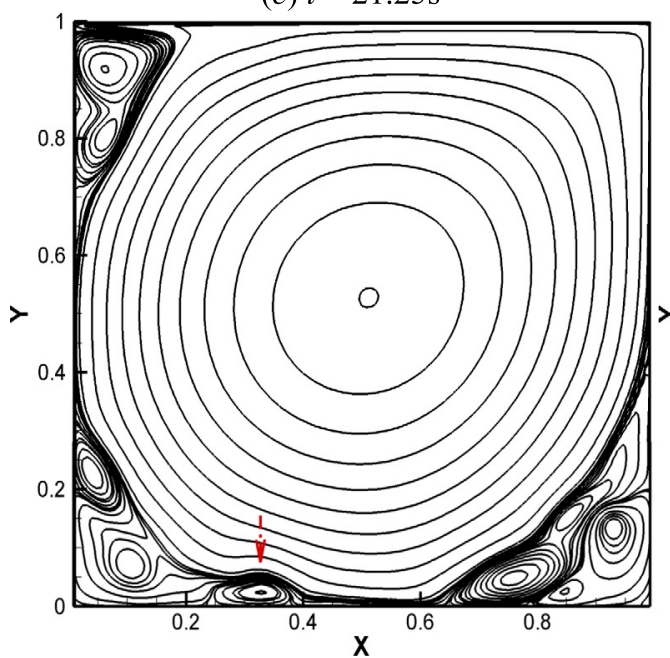

(e) $t=22.25 \mathrm{~s}$

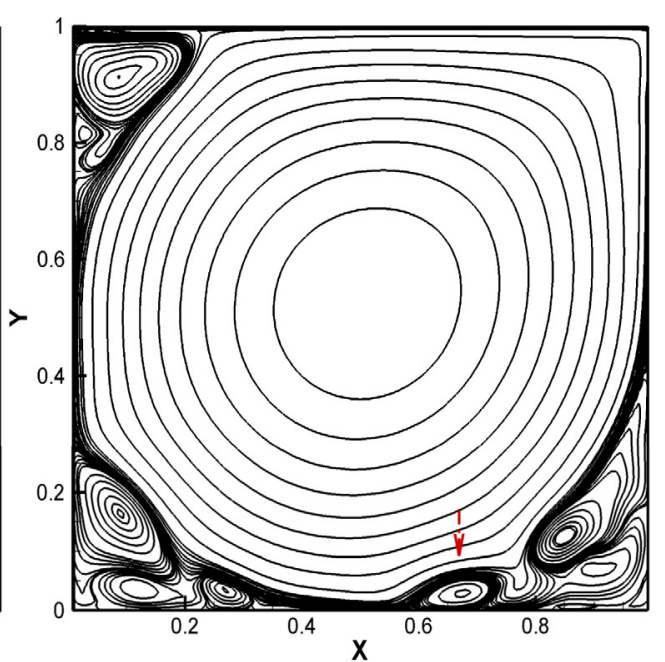

(b) $t=20.75 \mathrm{~s}$

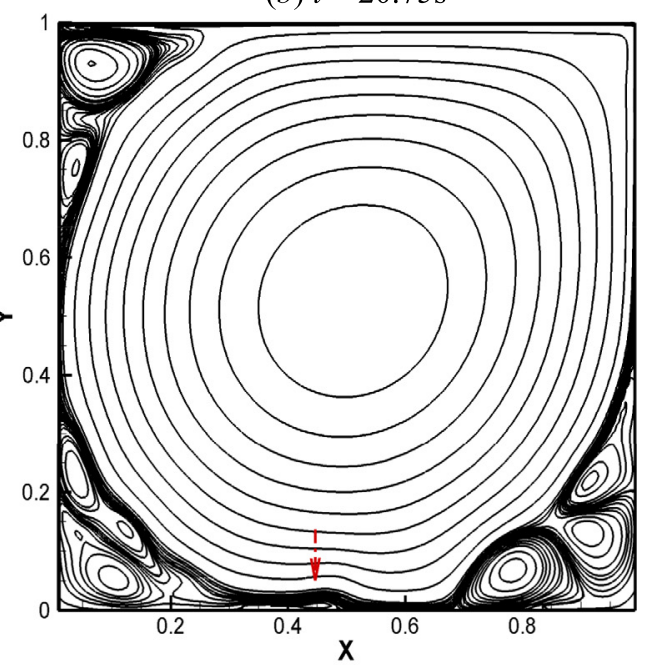

(d) $t=21.75 \mathrm{~s}$

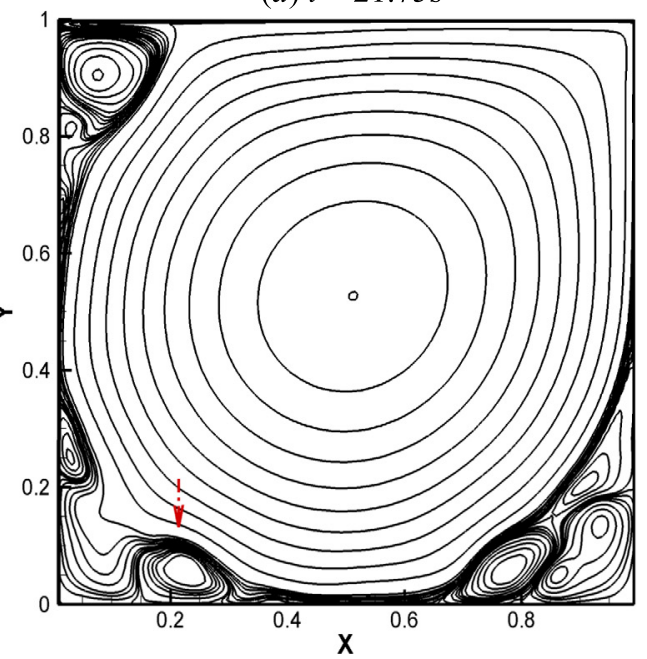

(f) $t=22.75 \mathrm{~s}$

Fig. 15. The stream function contours of the flow in a lid-driven cavity for $R e=20,000$ solved using the 5 th-order SUCS scheme and a uniform grid of size $(128 \times 128)$. A solitary vortex (denoted by the red arrow) can be observed shedding from the bottom-right corner and moving along the boundary to the bottom-left corner of the cavity. 


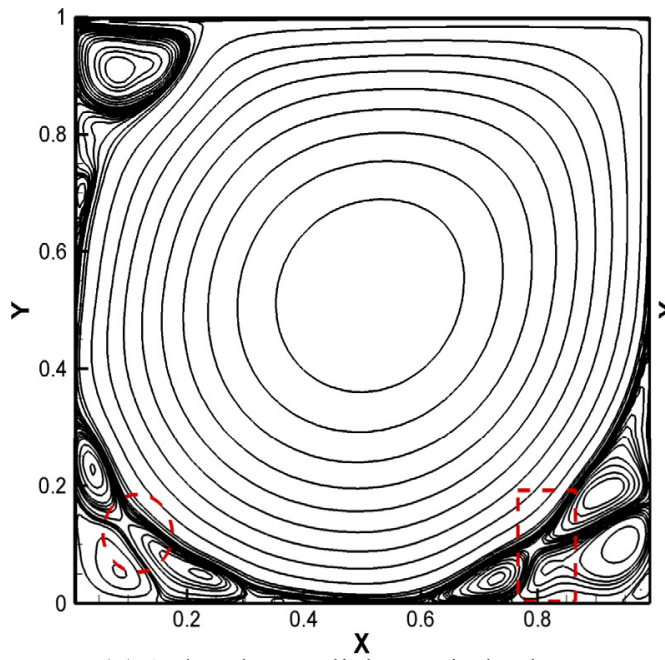

(a) 3rd-order explicit upwind scheme

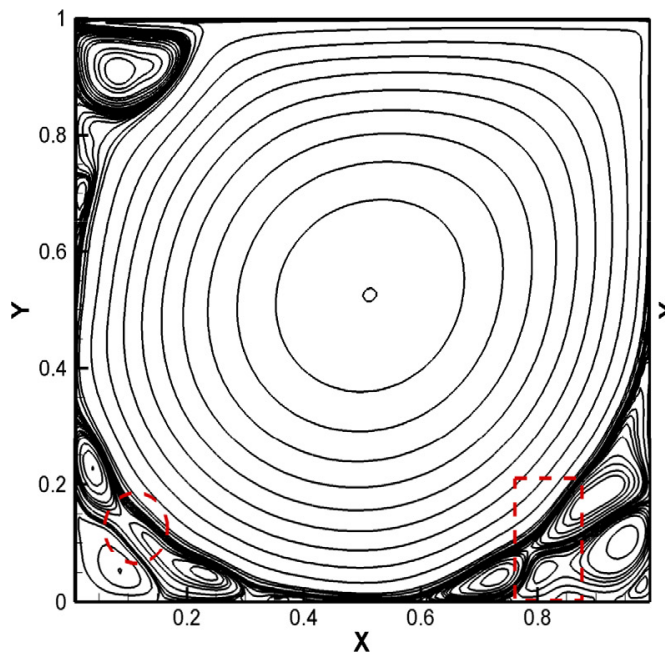

(c) 3rd-order SUCS scheme

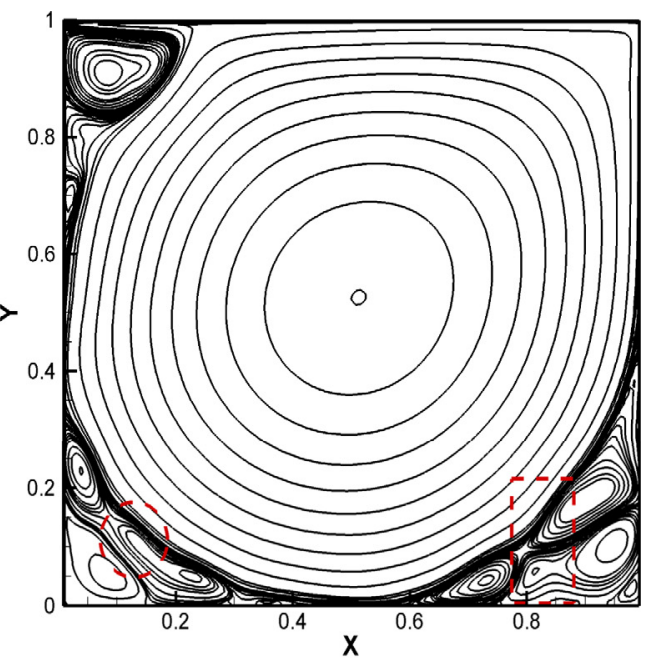

(b) 5th-order explicit upwind scheme

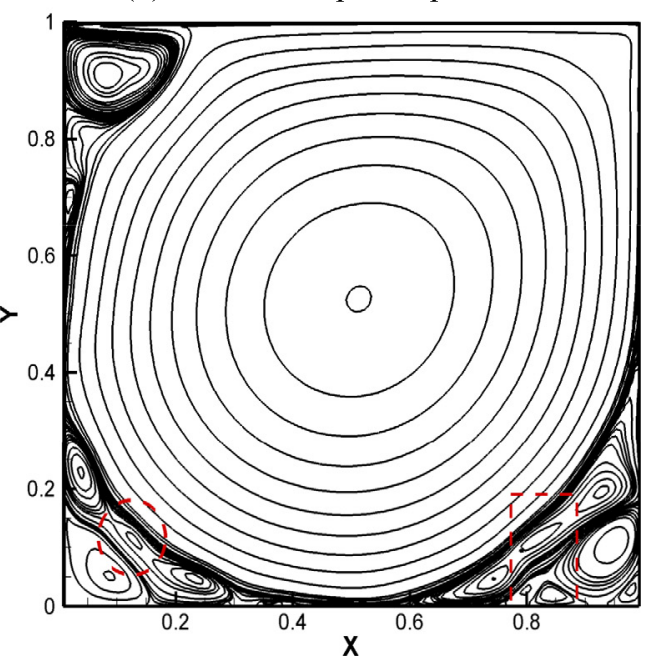

(d) 5th-order SUCS scheme

Fig. 16. The stream function contours of the flow in a lid-driven cavity at time $t=26 \mathrm{~s}$ with $R e=20,000$ and a uniform grid of size (128 $\times 128$ ), obtained by the 3rd-order explicit upwind scheme (a), 5th-order explicit upwind scheme (b), 3rd-order SUCS scheme (c) and 5th-order SUCS scheme (d).

with the leading order term given by

$$
e_{2 a}^{(2)}=-\frac{h_{-1}^{2}}{3 !}
$$

(ii) $\boldsymbol{B}_{\mathbf{2 b}}$. By using a similar procedure, another 2nd-order approximation $\boldsymbol{B}_{\mathbf{2}}$ based on stencil $\left\{\boldsymbol{u}_{-\mathbf{1}}^{\prime}, \boldsymbol{u}_{\mathbf{0}}, \boldsymbol{u}_{\mathbf{1}}\right\}$ can be obtained as well. The explicit form for $B_{2 b}$ is written as

$$
B_{2 b}=\frac{h_{1}}{h_{1}-2 h_{-1}} u_{-1}^{\prime}+\frac{-2 h_{-1}}{h_{1}-2 h_{-1}} \frac{u_{1}-u_{0}}{h_{1}}=u_{0}^{\prime}+\sum_{n=2}^{\infty} e_{2 b}^{(n)} u_{0}^{(n+1)}
$$

and coefficients of TE are given by

$$
e_{2 b}^{(n)}=\frac{h_{-1} h_{1}}{h_{1}-2 h_{-1}} \frac{(n+1) h_{-1}^{n-1}-2 h_{1}^{n-1}}{(n+1) !}
$$

(iii) $\boldsymbol{B}_{\mathbf{3}}$. With the previously obtained two 2nd-order approximations, $B_{2 a}$ and $B_{2 b}$, a 3rd-order approximation to $u_{0}^{\prime}, \boldsymbol{B}_{\mathbf{3}}$, based on stencil $\left\{\boldsymbol{u}_{-\mathbf{1}}^{\prime}, \boldsymbol{u}_{-\mathbf{1}}, \boldsymbol{u}_{\mathbf{0}}, \boldsymbol{u}_{\mathbf{1}}\right\}$ can be derived by using a similar weighted summation method, given by

$$
B_{3}=\omega B_{2 a}+(1-\omega) B_{2 b}=u_{0}^{\prime}+\sum_{n=3}^{\infty} e_{3}^{(n)} u_{0}^{(n+1)}
$$


where the weight can be calculated from

$$
\omega=\frac{e_{2 b}^{(2)}}{e_{2 b}^{(2)}-e_{2 a}^{(2)}}=\frac{h_{1}\left(3 h_{-1}-2 h_{1}\right)}{-2\left(h_{-1}-h_{1}\right)^{2}}
$$

By substituting (27), (30) and (33) into (32), the explicit form of $B_{3}$ can be obtained, written as

$$
B_{3}=\frac{h_{1} u_{-1}^{\prime}}{h_{-1}-h_{1}}-\frac{h_{1}}{h_{-1}-h_{1}}\left(2+\frac{h_{-1}}{h_{-1}-h_{1}}\right) \frac{u_{-1}-u_{0}}{h_{-1}}+\frac{h_{-1}^{2}}{\left(h_{1}-h_{-1}\right)^{2}} \frac{u_{1}-u_{0}}{h_{1}}
$$

and coefficients of the TE can be derived from

$$
\begin{aligned}
e_{3}^{(n)} & =\omega e_{2 a}^{(n)}+(1-\omega) e_{2 b}^{(n)} \\
& =\frac{h_{-1}^{2} h_{1}}{(n+1) !\left(h_{1}-h_{-1}\right)}\left[(1-n) h_{-1}^{n-2}+\frac{h_{1}^{n-1}-h_{-1}^{n-1}}{h_{1}-h_{-1}}\right] \\
& =\frac{h_{-1}^{2} h_{1}}{(n+1) !} \sum_{l_{1}=1}^{n-2} \sum_{l_{2}=0}^{l_{1}-1} h_{-1}^{n-3-l_{2}} h_{1}^{l_{2}}
\end{aligned}
$$

with leading order term given by

$$
e_{3}^{(3)}=\frac{h_{-1}^{2} h_{1}}{4 !}
$$

Note that Eq. (34) is a left-biased 3rd-order compact scheme which applies when the convection velocity is positive. The right-biased version based on stencil $\left\{\boldsymbol{u}_{\mathbf{1}}^{\prime}, \boldsymbol{u}_{-\mathbf{1}}, \boldsymbol{u}_{\mathbf{0}}, \boldsymbol{u}_{\mathbf{1}}\right\}$ can be obtained by exchanging subscripts 1 and -1 in Eq. (34), written by,

$$
B_{3 R}=\frac{h_{-1} u_{1}^{\prime}}{h_{1}-h_{-1}}-\frac{h_{-1}}{h_{1}-h_{-1}}\left(2+\frac{h_{1}}{h_{1}-h_{-1}}\right) \frac{u_{1}-u_{0}}{h_{1}}+\frac{h_{1}^{2}}{\left(h_{-1}-h_{1}\right)^{2}} \frac{u_{-1}-u_{0}}{h_{-1}}
$$

One can easily verify that in the uniform grids limit the coefficients in (34) and (37) agree with those presented in Tables 4a and $4 \mathrm{~b}$.

\subsubsection{4th-order of accuracy $\left(B_{4}\right)$}

The 4th-order approximation based on stencil $\left\{\boldsymbol{u}_{-1}^{\prime}, \boldsymbol{u}_{-\mathbf{1}}, \boldsymbol{u}_{\mathbf{0}}, \boldsymbol{u}_{\mathbf{1}}, \boldsymbol{u}_{\mathbf{2}}\right\}$ can also be derived from the previously used weighted summation method. First, we can separate this problem into two 3rd-order approximations, $B_{3}$ and $B_{3 b}$, based on stencils $\left\{u_{-1}^{\prime}, u_{-1}, u_{0}, u_{1}\right\}$ and $\left\{u_{-1}^{\prime}, u_{-1}, u_{0}, u_{2}\right\}$, respectively. The explicit expressions for $B_{3 b}$ and the corresponding TE $e_{3 b}$ can be obtained by just replacing subscript 1 in the expressions of $B_{3}$ and $e_{3}$ with 2, given by

$$
\begin{aligned}
& B_{3 b}=\frac{h_{2} u_{-1}^{\prime}}{h_{-1}-h_{1}}-\frac{h_{2}}{h_{-1}-h_{2}}\left(2+\frac{h_{-1}}{h_{-1}-h_{2}}\right) \frac{u_{-1}-u_{0}}{h_{-1}}+\frac{h_{-1}^{2}}{\left(h_{2}-h_{-1}\right)^{2}} \frac{u_{2}-u_{0}}{h_{2}} \\
& e_{3 b}^{(n)}=\frac{h_{-1}^{2} h_{2}}{(n+1) !} \sum_{l_{1}=1}^{n-2} \sum_{l_{2}=0}^{l_{1}-1} h_{-1}^{n-3-l_{2}} h_{2}^{l_{2}}
\end{aligned}
$$

Then, a 4th-order approximation to $u_{0}^{\prime}$ can be yielded from the following weighted summation operation

$$
B_{4}=\omega B_{3}+(1-\omega) B_{3 b}=u_{0}^{\prime}+\sum_{n=4}^{\infty} e_{4}^{(n)} u_{0}^{(n+1)}
$$

where the weight is calculated from

$$
\omega=\frac{e_{3 b}^{(3)}}{e_{3 b}^{(3)}-e_{3}^{(3)}}=\frac{h_{2}}{h_{2}-h_{1}}
$$

By substituting (34), (38) and (41) into (40), the explicit expression of $B_{4}$ can be acquired, given by

$$
\begin{aligned}
B_{4}= & \frac{-h_{1} h_{2} u_{-1}^{\prime}}{\left(h_{-1}-h_{1}\right)\left(h_{-1}-h_{2}\right)} \\
& +\frac{h_{1} h_{2}}{\left(h_{-1}-h_{1}\right)\left(h_{-1}-h_{2}\right)}\left[2+h_{-1}\left(\frac{1}{h_{-1}-h_{1}}+\frac{1}{h_{-1}-h_{2}}\right)\right] \frac{u_{-1}-u_{0}}{h_{-1}} \\
& +\frac{-h_{-1}^{2} h_{2}}{\left(h_{1}-h_{-1}\right)^{2}\left(h_{1}-h_{2}\right)} \frac{u_{1}-u_{0}}{h_{1}}+\frac{-h_{-1}^{2} h_{1}}{\left(h_{2}-h_{-1}\right)^{2}\left(h_{2}-h_{1}\right)} \frac{u_{2}-u_{0}}{h_{2}}
\end{aligned}
$$


and coefficients of the TE can be derived from

$$
\begin{aligned}
e_{4}^{(n)} & =\omega e_{3}^{(n)}+(1-\omega) e_{3 b}^{(n)} \\
& =\frac{-h_{-1}^{2} h_{1} h_{2}}{(n+1) !} \sum_{l_{1}=1}^{n-2} \sum_{l_{2}=0}^{l_{1}-1} h_{-1}^{n-3-l_{2}} \frac{h_{2}^{l_{2}}-h_{1}^{l_{2}}}{h_{2}-h_{1}} \\
& =\frac{-h_{-1}^{2} h_{1} h_{2}}{(n+1) !} \sum_{l_{1}=2}^{n-2} \sum_{l_{2}=1}^{l_{1}-1} \sum_{l_{3}=0}^{l_{2}-1} h_{-1}^{n-3-l_{2}} h_{1}^{l_{2}-1-l_{3}} h_{2}^{l_{3}}
\end{aligned}
$$

with the leading order term given by

$$
e_{4}^{(4)}=\frac{-h_{-1}^{2} h_{1} h_{2}}{5 !}
$$

If a right running wave is to be examined using the above 4th-order bi-diagonal scheme, the left-biased version of Eq. (42) based on stencil $\left\{\boldsymbol{u}_{-\mathbf{1}}^{\prime}, \boldsymbol{u}_{-\mathbf{2}}, \boldsymbol{u}_{-\mathbf{1}}, \boldsymbol{u}_{\mathbf{0}}, \boldsymbol{u}_{\mathbf{1}}\right\}$ can be used, which can be obtained by replacing subscript 2 in Eq. (42) with -2 , given by,

$$
\begin{aligned}
B_{4 L}= & \frac{-h_{1} h_{-2} u_{-1}^{\prime}}{\left(h_{-1}-h_{1}\right)\left(h_{-1}-h_{-2}\right)} \\
& +\frac{h_{1} h_{-2}}{\left(h_{-1}-h_{1}\right)\left(h_{-1}-h_{-2}\right)}\left[2+h_{-1}\left(\frac{1}{h_{-1}-h_{1}}+\frac{1}{h_{-1}-h_{-2}}\right)\right] \frac{u_{-1}-u_{0}}{h_{-1}} \\
& +\frac{-h_{-1}^{2} h_{-2}}{\left(h_{1}-h_{-1}\right)^{2}\left(h_{1}-h_{-2}\right)} \frac{u_{1}-u_{0}}{h_{1}}+\frac{-h_{-1}^{2} h_{1}}{\left(h_{-2}-h_{-1}\right)^{2}\left(h_{-2}-h_{1}\right)} \frac{u_{-2}-u_{0}}{h_{-2}}
\end{aligned}
$$

\subsection{3. $p$ th-order of accuracy $\left(B_{p}\right)(p \geq 3)$}

To derive the results for arbitrary order of accuracy, we can first assume a general form for the arbitrary $p$ th-order $(p \geq 3)$ approximation by inspecting the common characteristics from the previously obtained 3rd-order and 4th-order results. According to the explicit expressions of $B_{3}$ and $B_{4}$, the $p$ th-order approximation $\boldsymbol{B}_{\boldsymbol{p}}$ based on stencil $\left\{\boldsymbol{u}_{-\mathbf{1}}^{\prime}, \boldsymbol{u}_{-\mathbf{1}}, \boldsymbol{u}_{\mathbf{0}}, \boldsymbol{u}_{\mathbf{1}}, \ldots\right.$, $\left.\boldsymbol{u}_{\boldsymbol{p}-\mathbf{3}}, \boldsymbol{u}_{\boldsymbol{p}-\mathbf{2}}\right\}$ can be assumed written as

$$
B_{p}=-\sum_{i \in \Omega_{1}} \alpha_{i} u_{i}^{\prime}+\sum_{j \in \Omega_{1} \cup \Omega_{2}} c_{j} \frac{u_{j}-u_{0}}{h_{j}}=u_{0}^{\prime}+\sum_{n=p}^{\infty} e_{p}^{(n)} u_{0}^{(n+1)}
$$

where the two point sets are

$$
\Omega_{1} \equiv\{-1\} ; \quad \Omega_{2} \equiv[1, p-2]
$$

and the coefficients are

$$
\begin{aligned}
& \left.\alpha_{i}\right|_{i \in \Omega_{1}}=(-1)^{p} \prod_{j \in \Omega_{2}} \frac{h_{j}}{h_{i}-h_{j}} \\
& c_{j}= \begin{cases}(-1)^{p} \prod_{k \in \Omega_{2}} \frac{h_{k}}{h_{j}-h_{k}}\left(2+\sum_{l \in \Omega_{2}} \frac{h_{j}}{h_{j}-h_{l}}\right), \quad j \in \Omega_{1} \\
(-1)^{p+1} \prod_{k \in \Omega_{1}} \frac{h_{k}^{2}}{\left(h_{j}-h_{k}\right)^{2}} \prod_{l \in \Omega_{2}, l \neq j} \frac{h_{l}}{h_{j}-h_{l}}, \quad j \in \Omega_{2}\end{cases}
\end{aligned}
$$

Similarly, according to the explicit forms of $e_{3}$ and $e_{4}$, the TE of $B_{p}$ can be assumed expressed as

$$
e_{p}^{(n)}=(-1)^{p+1} \frac{\prod_{i \in \Omega_{1}} h_{i}^{2} \prod_{j \in \Omega_{2}} h_{j}}{(n+1) !} \sum_{l_{1}=p-2}^{n-2} \sum_{l_{2}=p-3}^{l_{1}-1} \ldots \sum_{l_{p-1}=0}^{l_{p-2}-1} h_{-1}^{n-3-l_{2}} h_{1}^{l_{2}-1-l_{3}} \cdots h_{p-3}^{l_{p-2}-1-l_{p-1}} h_{p-2}^{l_{p-1}}
$$

with the leading order term given by

$$
e_{p}^{(p)}=(-1)^{p+1} \frac{\prod_{i \in \Omega_{1}} h_{i}^{2} \prod_{j \in \Omega_{2}} h_{j}}{(p+1) !}
$$


5.1.4. $(p+1)$ th-order of accuracy $\left(B_{p+1}\right)(p \geq 3)$

Based on the previously assumptions for the $p$ th-order approximation, the $(p+1)$ th-order approximation $\boldsymbol{B}_{\boldsymbol{p}+\mathbf{1}}$ based on stencil $\left\{\boldsymbol{u}_{-\mathbf{1}}^{\prime}, \boldsymbol{u}_{-\mathbf{1}}, \boldsymbol{u}_{\mathbf{0}}, \boldsymbol{u}_{\mathbf{1}}, \ldots, \boldsymbol{u}_{\boldsymbol{p}-\mathbf{2}}, \boldsymbol{u}_{\boldsymbol{p}-\mathbf{1}}\right\}$ can be derived by the following weighted summation method. We first replace subscript $p-2$ in $B_{p}$ and $e_{p}$ with $p-1$ to obtain another $p$ th-order approximation $B_{p b}$ based on stencil $\left\{u_{-1}^{\prime}, u_{-1}, u_{0}, u_{1}, \ldots, u_{p-3}, u_{p-1}\right\}$. The explicit expressions of $B_{p b}$ and the corresponding TE $e_{p b}$ are given by

$$
\begin{aligned}
B_{p b} & =-\sum_{i \in \Omega_{1}} \alpha_{i} u_{i}^{\prime}+\sum_{j \in \Omega_{1} \cup \Omega_{2}} c_{j} \frac{u_{j}-u_{0}}{h_{j}} \\
e_{p b}^{(n)} & =(-1)^{p+1} \frac{h_{-1}^{2} h_{p-1} \prod_{i=1}^{p-3} h_{i}}{(n+1) !} \sum_{l_{1}=p-2}^{n-2} \sum_{l_{2}=p-3}^{l_{1}-1} \cdots \sum_{l_{p-1}=0}^{l_{p-2}-1} h_{-1}^{n-3-l_{2}} h_{1}^{l_{2}-1-l_{3}} \cdots h_{p-3}^{l_{p-2}-1-l_{p-1}} h_{p-1}^{l_{p-1}}
\end{aligned}
$$

In (52) the coefficients $\alpha_{i}$ and $c_{j}$ have the same forms as those in (48) and (49) except the two sets change to

$$
\Omega_{1} \equiv\{-1\} ; \quad \Omega_{2} \equiv[1, p-3] \cup\{p-1\}
$$

The $(p+1)$ th-order approximation to $u_{0}^{\prime}, B_{p+1}$, can then be yielded from the following weighted summation operation

$$
B_{p+1}=\omega B_{p}+(1-\omega) B_{p b}=u_{0}^{\prime}+\sum_{n=p+1}^{\infty} e_{p+1}^{(n)} u_{0}^{(n+1)}
$$

where the weight is evaluated from

$$
\omega=\frac{e_{p b}^{(p)}}{e_{p b}^{(p)}-e_{p}^{(p)}}=\frac{h_{p-1}}{h_{p-1}-h_{p-2}}
$$

By substituting (46), (52) and (56) into (55), the explicit form for $B_{p+1}$ can be derived and written as

$$
B_{p+1}=-\sum_{i \in \Omega_{1}} \alpha_{i} u_{i}^{\prime}+\sum_{j \in \Omega_{1} \cup \Omega_{2}} c_{j} \frac{u_{j}-u_{0}}{h_{j}}
$$

where

$$
\Omega_{1} \equiv\{-1\} ; \quad \Omega_{2} \equiv[1, p-1]
$$

The coefficients $\alpha_{i}$ and $c_{j}$ in (57) have the same forms as those in (48) and (49) except $p$ is replaced by $p+1$. The coefficients of TE can be derived from

$$
\begin{aligned}
e_{p+1}^{(n)} & =\omega e_{p}^{(n)}+(1-\omega) e_{p b}^{(n)} \\
& =(-1)^{p+2} \frac{h_{-1}^{2} \prod_{i=1}^{p-1} h_{i}}{(n+1) !} \sum_{l_{1}=p-2}^{n-2} \sum_{l_{2}=p-3}^{l_{1}-1} \cdots \sum_{l_{p-1}=0}^{l_{p-2}-1} h_{-1}^{n-3-l_{2}} h_{1}^{l_{2}-1-l_{3}} \cdots h_{p-3}^{l_{p-2}-1-l_{p-1}} \frac{h_{p-1}^{l_{p-1}}-h_{p-2}^{l_{p-1}}}{h_{p-1}-h_{p-2}}
\end{aligned}
$$

which can be further simplified as

$$
e_{p+1}^{(n)}=(-1)^{p+2} \frac{\prod_{i \in \Omega_{1}} h_{i}^{2} \prod_{j \in \Omega_{2}} h_{j}}{(n+1) !} \sum_{l_{1}=p-1}^{n-2} \sum_{l_{2}=p-2}^{l_{1}-1} \ldots \sum_{l_{p-1}=1}^{l_{p-2}-1} \sum_{l_{p}=0}^{l_{p-1}-1} h_{-1}^{n-3-l_{2}} h_{1}^{l_{2}-1-l_{3}} \cdots h_{p-2}^{l_{p-1}-1-l_{p}} h_{p-1}^{l_{p}}
$$

The leading order term taken at $n=p+1$ has the same form as (51) except $p$ replaced by $p+1$. With the observation that the general form of the bi-diagonal scheme postulated in section 5.1.3 is valid for $p=3$ (section 5.1.1.iii) and $p=4$ (section 5.1.2), and the application of the mathematical induction principle, section 5.1.4 constitutes a formal proof that the form in section 5.1 .3 is valid for all integer $p \geq 3$.

\subsubsection{Boundary closure scheme}

The boundary closure schemes (with one more unknown derivative variable postulated at the point adjacent to the boundary) can be derived directly from the results obtained previously. To obtain arbitrary pth-order boundary closure scheme based on stencil $\left\{\boldsymbol{u}_{\mathbf{1}}^{\prime}, \boldsymbol{u}_{\mathbf{0}}, \boldsymbol{u}_{\mathbf{1}}, \boldsymbol{u}_{\mathbf{2}}, \ldots, \boldsymbol{u}_{\boldsymbol{p}-\mathbf{1}}\right\}$, one can first exchange subscripts 1 and -1 in Eq. (46) and then replace the subscript -1 with $p-1$. After doing so, the following general form for the left boundary scheme can be obtained,

$$
C_{p L}=-\alpha_{1} u_{1}^{\prime}+\sum_{j=1}^{p-1} c_{j} \frac{u_{j}-u_{0}}{h_{j}}
$$


where the coefficients are

$$
\begin{aligned}
\alpha_{1} & =\prod_{j=2}^{p-1} \frac{h_{j}}{h_{j}-h_{1}} \\
c_{j} & =\left\{\begin{array}{l}
\prod_{k=2}^{p-1} \frac{h_{k}}{h_{k}-h_{1}}\left(2-\sum_{l=2}^{p-1} \frac{h_{1}}{h_{l}-h_{1}}\right), \quad j=1 \\
\frac{h_{1}^{2}}{\left(h_{j}-h_{1}\right)^{2}} \prod_{\substack{l=2, l \neq j}}^{p-1} \frac{h_{l}}{h_{l}-h_{j}}, \quad j \in[2, p-1]
\end{array}\right.
\end{aligned}
$$

The leading term of the truncation is given by

$$
e_{p}^{(p)}=(-1)^{p+1} \frac{h_{1} \prod_{j=1}^{p-1} h_{j}}{(p+1) !}
$$

Under the uniform grids assumption the above results reduce to,

$$
C_{p L}=-\alpha_{1} u_{1}^{\prime}+\sum_{j=1}^{p-1} c_{j}\left(u_{j}-u_{0}\right)
$$

in which,

$$
\begin{aligned}
& \alpha_{1}=p-1 \\
& c_{1}=(p-1)\left(2-\sum_{l=1}^{p-2} \frac{1}{l}\right), \\
& c_{j}=\frac{(-1)^{j}}{j(j-1)} \frac{1}{j !} \frac{(p-1) !}{(p-1-j) !}, \quad j \in[2, p-1]
\end{aligned}
$$

and the leading term of the truncation error becomes

$$
e_{p}^{(p)}=\frac{(-1)^{p+1} h^{p}}{(p+1) !}
$$

The explicit form for the right boundary closure scheme can be obtained simply by noting the symmetrical and antisymmetrical features of the coefficients, given by

$$
C_{p R}=-\alpha_{-1} u_{-1}^{\prime}+\sum_{j=0}^{p-1} c_{-j} u_{-j}
$$

where

$$
\begin{aligned}
& \alpha_{-1}=\alpha_{1}, \\
& c_{-j}=-c_{j}, \quad j \in[0, p-1]
\end{aligned}
$$

\subsection{Tri-diagonal $T_{p+1}[(p+1)$ th-order $(p \geq 3)]$}

Problems that contain more than two unknown derivative values can also be disentangled favorably from the previously used weighted summation method. For example, considering a tri-diagonal compact scheme $\boldsymbol{T}_{\boldsymbol{p}+\mathbf{1}}$ that is $(p+1)$ th-order accurate and based on stencil $\left\{\boldsymbol{u}_{-\mathbf{1}}^{\prime}, \boldsymbol{u}_{\mathbf{1}}^{\prime}, \boldsymbol{u}_{-\mathbf{1}}, \boldsymbol{u}_{\mathbf{0}}, \boldsymbol{u}_{\mathbf{1}}, \ldots, \boldsymbol{u}_{\boldsymbol{p}-\mathbf{3}}, \boldsymbol{u}_{\boldsymbol{p}-\mathbf{2}}\right\}$. We can separate this problem into two $p$ th-order bi-diagonal schemes, $B_{p}$ and $B_{p c}$, based on stencils $\left\{u_{-1}^{\prime}, u_{-1}, u_{0}, u_{1}, \ldots, u_{p-3}, u_{p-2}\right\}$ and $\left\{u_{1}^{\prime}, u_{-1}, u_{0}, u_{1}, \ldots, u_{p-3}, u_{p-2}\right\}$, respectively. The explicit formulations for $B_{p}$ and the corresponding TE $e_{p}$ have been given in section 5.1.3, while, those for $B_{p c}$ and the corresponding TE $e_{p c}$ can be obtained just by exchanging subscripts 1 and -1 in $B_{p}$ and $e_{p}$, respectively, given by

$$
\begin{aligned}
B_{p c} & =-\sum_{i \in \Omega_{1}} \alpha_{i} u_{i}^{\prime}+\sum_{j \in \Omega_{1} \cup \Omega_{2}} c_{j} \frac{u_{j}-u_{0}}{h_{j}} \\
e_{p c}^{(n)} & =(-1)^{p+1} \frac{h_{1}^{2} h_{-1} \prod_{i=2}^{p-2} h_{i}}{(n+1) !} \sum_{l_{1}=p-2}^{n-2} \sum_{l_{2}=p-3}^{l_{1}-1} \cdots \sum_{l_{p-1}=0}^{l_{p-2}-1} h_{1}^{n-3-l_{2}} h_{-1}^{l_{2}-1-l_{3}} \cdots h_{p-3}^{l_{p-2}-1-l_{p-1}} h_{p-2}^{l_{p-1}}
\end{aligned}
$$


In (70) the coefficients $\alpha_{i}$ and $c_{j}$ have the same forms as those in (48) and (49) except the two sets change to

$$
\Omega_{1} \equiv\{1\} ; \quad \Omega_{2} \equiv[2, p-2] \cup\{-1\}
$$

The $(p+1)$ th-order approximation to $u_{0}^{\prime}, T_{p+1}$, can be yielded from the following weighted summation operation,

$$
T_{p+1}=\omega B_{p}+(1-\omega) B_{p c}=u_{0}^{\prime}+\sum_{n=p+1}^{\infty} e_{p+1}^{(n)} u_{0}^{(n+1)}
$$

where the weight is

$$
\omega=\frac{e_{p c}^{(p)}}{e_{p c}^{(p)}-e_{p}^{(p)}}=\frac{h_{1}}{h_{1}-h_{-1}}
$$

By substituting (46), (70) and (74) into (73), the explicit form for $T_{p+1}$ can be given by

$$
T_{p+1}=-\sum_{i \in \Omega_{1}} \alpha_{i} u_{i}^{\prime}+\sum_{j \in \Omega_{1} \cup \Omega_{2}} c_{j} \frac{u_{j}-u_{0}}{h_{j}}
$$

The two sets are

$$
\Omega_{1} \equiv\{-1,1\} ; \quad \Omega_{2} \equiv[2, p-2]
$$

and the coefficients are

$$
\begin{aligned}
& \left.\alpha_{i}\right|_{i \in \Omega_{1}}=(-1)^{p+1} \prod_{k \in \Omega_{1}, k \neq i} \frac{h_{k}^{2}}{\left(h_{i}-h_{k}\right)^{2}} \prod_{l \in \Omega_{2}} \frac{h_{l}}{h_{i}-h_{l}} \\
& c_{j}=\left\{\begin{array}{l}
(-1)^{p+1}\left(2+\sum_{k \in \Omega_{1}, k \neq j} \frac{2 h_{j}}{h_{j}-h_{k}}+\sum_{l \in \Omega_{2}} \frac{h_{j}}{h_{j}-h_{l}}\right) \prod_{k \in \Omega_{1}, k \neq j} \frac{h_{k}^{2}}{\left(h_{j}-h_{k}\right)^{2}} \prod_{l \in \Omega_{2}} \frac{h_{l}}{h_{j}-h_{l}}, \quad j \in \Omega_{1} \\
(-1)^{p+2} \prod_{k \in \Omega_{1}} \frac{h_{k}^{2}}{\left(h_{j}-h_{k}\right)^{2}} \prod_{l \in \Omega_{2}, l \neq j} \frac{h_{l}}{h_{j}-h_{l}}, \quad j \in \Omega_{2}
\end{array}\right.
\end{aligned}
$$

Coefficients of TE of $T_{p+1}$ can be derived from

$$
\begin{aligned}
e_{p+1}^{(n)}= & \omega e_{p}^{(n)}+(1-\omega) e_{p c}^{(n)} \\
= & (-1)^{p+2} \frac{\prod_{i \in \Omega_{1}} h_{i}^{2} \prod_{j \in \Omega_{2}} h_{j}}{(n+1) !} \times \\
& \sum_{l_{1}=p-2}^{n-3} \sum_{l_{2}=p-3}^{l_{1}-1} \cdots \sum_{l_{p-1}=0}^{l_{p-2}-1} \sum_{l_{p}=0}^{n-3-2 l_{2}+l_{3}} h_{-1}^{n-4-l_{2}-l_{p}} h_{1}^{l_{2}-1-l_{3}+l_{p}} h_{2}^{l_{3}-1-l_{4}} \cdots h_{p-3}^{l_{p-2}-1-l_{p-1}} h_{p-2}^{l_{p-1}}
\end{aligned}
$$

It is clearly seen from the above equation that at $n=p+1$

$$
\left\{\begin{array}{l}
l_{1}=p-2, l_{2}=p-3, \cdots, l_{p-1}=0 \\
l_{p}=0
\end{array}, \quad n=p+1\right.
$$

which indicates the leading order term taken at $n=p+1$ has the same form as (51) except $p$ is replaced by $p+1$.

For the simplest case among the tri-diagonal category, the 5th-order right-biased SUCS scheme based on stencil $\left\{\boldsymbol{u}_{-\mathbf{1}}^{\prime}, \boldsymbol{u}_{\mathbf{1}}^{\prime}, \boldsymbol{u}_{-\mathbf{1}}, \boldsymbol{u}_{\mathbf{0}}, \boldsymbol{u}_{\mathbf{1}}, \boldsymbol{u}_{\mathbf{2}}\right\}$ can be obtained straightforwardly by substituting $p=4$ into (75)-(78). The coefficients are

$$
\begin{aligned}
& \alpha_{-1}=\frac{-h_{1}^{2}}{\left(h_{-1}-h_{1}\right)^{2}} \frac{h_{2}}{h_{-1}-h_{2}} ; \\
& \alpha_{1}=\frac{-h_{-1}^{2}}{\left(h_{1}-h_{-1}\right)^{2}} \frac{h_{2}}{h_{1}-h_{2}} \\
& c_{-1}=\frac{-1}{h_{-1}} \frac{h_{1}^{2}}{\left(h_{-1}-h_{1}\right)^{2}} \frac{h_{2}}{h_{-1}-h_{2}}\left(2+\frac{2 h_{-1}}{h_{-1}-h_{1}}+\frac{h_{-1}}{h_{-1}-h_{2}}\right), \\
& c_{1}=\frac{-1}{h_{1}} \frac{h_{-1}^{2}}{\left(h_{1}-h_{-1}\right)^{2}} \frac{h_{2}}{h_{1}-h_{2}}\left(2+\frac{2 h_{1}}{h_{1}-h_{-1}}+\frac{h_{1}}{h_{1}-h_{2}}\right), \\
& c_{2}=\frac{1}{h_{2}} \frac{h_{-1}^{2}}{\left(h_{2}-h_{-1}\right)^{2}} \frac{h_{1}^{2}}{\left(h_{2}-h_{1}\right)^{2}}
\end{aligned}
$$


Under the uniform grids assumption the above coefficients reduce to those listed in Table 4b. The explicit form for the 5th-order left-biased SUCS scheme based on stencil $\left\{\boldsymbol{u}_{-\mathbf{1}}^{\prime}, \boldsymbol{u}_{\mathbf{1}}^{\prime}, \boldsymbol{u}_{-\mathbf{2}}, \boldsymbol{u}_{-\mathbf{1}}, \boldsymbol{u}_{\mathbf{0}}, \boldsymbol{u}_{\mathbf{1}}\right\}$ is analogous to the above result except that the point set $\Omega_{2}$ should be replaced by $\Omega_{2} \equiv\{-2\}$.

For the more general $(2 r+1)$ th-order case, the right-biased SUCS scheme based on stencil $\left\{\boldsymbol{u}_{-1}^{\prime}, \boldsymbol{u}_{\mathbf{1}}^{\prime}, \boldsymbol{u}_{\mathbf{1}}-\boldsymbol{r}, \ldots, \boldsymbol{u}_{\mathbf{r}}\right\}$ can be obtained by first taking $p=2 r$ into (75)-(78) and then replacing subscripts $r+1, \ldots, 2 r-2$ with $1-r, \ldots,-2$, respectively. The point sets are

$$
\Omega_{1} \equiv\{-1,1\} ; \quad \Omega_{2} \equiv[1-r,-2] \cup[2, r]
$$

The left-biased version of the above scheme based on stencil $\left\{\boldsymbol{u}_{-\mathbf{1}}^{\prime}, \boldsymbol{u}_{\mathbf{1}}^{\prime}, \boldsymbol{u}_{-\boldsymbol{r}}, \ldots, \boldsymbol{u}_{\boldsymbol{r}-\mathbf{1}}\right\}$ has an analogous form except that the point set $\Omega_{2}$ changes to

$$
\Omega_{2} \equiv[-r,-2] \cup[2, r-1]
$$

Note that the derivations and results for the quarter-diagonal and penta-diagonal compact schemes are quite similar as those presented for the tri-diagonal compact schemes. For sake of conciseness, we don't present the complete derivations for these cases.

Furthermore, it has been mentioned previously that the basic numerical features of a difference scheme can be analyzed directly from the TE. For a better clarification, we consider, for example, a 5th-order tri-diagonal upwind compact scheme, $T_{5}$, based on stencil $\left\{u_{-1}^{\prime}, u_{1}^{\prime}, u_{-1}, u_{0}, u_{1}, u_{2}\right\}$. From substituting $p=4$ into (51), one can find that the leading term of TE of $T_{5}$ is $h_{-1}^{2} h_{1}^{2} h_{2} / 6$ !, manifesting $T_{5}$ is 5th-order accurate. On the other hand, one can notice from (73) that the leading term of TE of $T_{5}$ is linearly proportional to $u_{0}^{(6)}$, indicating the scheme denoted by $T_{5}$ is dissipative. This property can be easily understood from a simple Fourier analysis. Consider a small disturbance characterized by $u_{0}=e^{i k x}$, where $k$ is the wave number. By evaluating 6th-derivative of $e^{i k x}$, one can obtain that the amplification factor of this small disturbance is written by $G=(i k)^{6}=-k^{6}$, from which the dissipative property of the scheme is evident. Similarly, one can also find that the amplification factor of any even order central compact scheme $T_{2 m}$ can be expressed as $G=(i k)^{2 m+1}$, indicating that all of the central compact scheme is dispersive.

\section{Conclusions}

New upwind compact schemes termed as SUCS are presented for solving the incompressible Navier-Stokes equations. In contrast to the existing OUCS schemes, the newly developed SUCS schemes are constructed based on upwind-biased stencils as does the classical upwind schemes and can achieve sufficient numerical dissipation to stabilize the numerical solutions without the need of carrying out the optimization calculations. The SUCS are more compact than the OUCS because orders of accuracy of SUCS attain their maximum achievable values while those of OUCS do not.

The SUCS schemes have prominent advantages on combining the stable and high resolution properties which are demonstrated from the global spectral analyses and typical numerical experiments. From simulating the 1-D linear wave equation with discontinuous initial condition the good stable property of SUCS are substantiated. Furthermore, the high resolution capability of SUCS is exhibited through simulating the classical lid-driven cavity flows especially for the high Reynolds number cases $(R e=20,000)$.

Finally, the generalized formulations of SUCS are derived by using the stepwise weighted summation method and the mathematical induction principle. As a chief benefit of this method, truncation errors can be derived concurrently with the fully explicit presentations of the coefficients of the schemes. This enables directly delineating the basic numerical features of the schemes.

\section{Acknowledgements}

This work is supported by NSFC (11172299).

\section{References}

[1] D.X. Fu, Y.W. Ma, A high order accurate difference scheme for complex flow fields, J. Comput. Phys. 134 (1997) 1-15.

[2] H.W. Sun, L.Z. Li, A CCD-ADI method for unsteady convection-diffusion equations, Comput. Phys. Commun. 185 (2014) $790-797$.

[3] K. Zhou, S.H. Ni, Z.F. Tian, Exponential high-order compact scheme on nonuniform grids for the steady MHD duct flow problems with high Hartmann numbers, Comput. Phys. Commun. 196 (2015) 194-211.

[4] S.S. Xie, G.X. Li, S. Yi, Compact finite difference schemes with high accuracy for one-dimensional nonlinear Schrödinger equation, Comput. Methods Appl. Mech. Eng. 198 (2009) 1052-1060.

[5] A. Mohebbi, M. Abbaszadeh, M. Dehghan, Compact finite difference scheme and RBF meshless approach for solving 2D Rayleigh-Stokes problem for a heated generalized second grade fluid with fractional derivatives, Comput. Methods Appl. Mech. Eng. 264 (2013) $163-177$.

[6] S.K. Lele, Compact finite difference schemes with spectral-like resolution, J. Comput. Phys. 103 (1992) 16-42.

[7] P.C. Chu, C. Fan, A three-point combined compact difference scheme, J. Comput. Phys. 140 (1998) 370-399.

[8] P.C. Chu, C. Fan, A three-point sixth-order nonuniform combined compact difference scheme, J. Comput. Phys. 148 (1999) 663-674.

[9] R.K. Shukla, X. Zhong, Derivation of high-order compact finite difference schemes for non-uniform grid using polynomial interpolation, J. Comput. Phys. 204 (2005) 404-429. 
[10] T.K. Sengupta, V. Lakshmanan, V.V.S.N. Vijay, A new combined stable and dispersion relation preserving compact scheme for non-periodic problems, J. Comput. Phys. 228 (2009) 3048-3071.

[11] T.K. Sengupta, V.V.S.N. Vijay, S. Bhaumik, Further improvement and analysis of CCD scheme: dissipation discretization and de-aliasing properties, J. Comput. Phys. 228 (2009) 6150-6168.

[12] X. Zhong, High-order finite-difference schemes for numerical solution of hypersonic boundary-layer transition, J. Comput. Phys. 144 (1998) 662-709.

[13] T.K. Sengupta, G. Ganeriwal, S. De, Analysis of central and upwind compact schemes, J. Comput. Phys. 192 (2003) 677-694.

[14] A.K. De, V. Eswaran, Analysis of a new high resolution upwind compact scheme, J. Comput. Phys. 218 (2006) 398-416.

[15] Y.G. Bhumkar, T.W.H. Sheu, T.K. Sengupta, A dispersion relation preserving optimized upwind compact difference scheme for high accuracy flow simulations, J. Comput. Phys. 278 (2014) 378-399.

[16] T.K. Sengupta, A. Dipankar, P. Sagaut, Error dynamics: beyond von Neumann analysis, J. Comput. Phys. 226 (2007) 1211-1218.

[17] W. Chen, J.C. Chen, E.Y. Lo, An interpolation based finite difference method on non-uniform grid for solving Navier-Stokes equations, Comput. Fluids 101 (2014) 273-290.

[18] J.B. Bell, P. Colella, H.M. Glaz, A second-order projection method for the incompressible Navier-Stokes equations, J. Comput. Phys. 85 (1989) 257-283.

[19] D.L. Brown, R. Cortez, M.L. Minion, Accurate projection method for the incompressible Navier-Stokes equations, J. Comput. Phys. 168 (2001) 464-499.

[20] J. Liu, Open and traction boundary conditions for the incompressible Navier-Stokes equations, J. Comput. Phys. 228 (2009) $7250-7267$.

[21] J.-G. Liu, J. Liu, R.L. Pego, Stable and accurate pressure approximation for unsteady incompressible viscous flow, J. Comput. Phys. 229 (2010) $3428-3453$.

[22] D. Shirokoff, R.R. Rosales, An efficient method for the incompressible Navier-Stokes equations on irregular domains with no-slip boundary conditions, high order up to the boundary, J. Comput. Phys. 230 (2011) 8619-8646.

[23] S. Dong, G.E. Karniadakis, C. Chryssostomidis, A robust and accurate outflow boundary condition for incompressible flow simulations on severelytruncated unbounded domains, J. Comput. Phys. 261 (2014) 83-105.

[24] A.W. Vreman, The projection method for the incompressible Navier-Stokes equations: the pressure near a no-slip wall, J. Comput. Phys. 263 (2014) 353-374.

[25] P.N. Shankar, M.D. Deshpande, Fluid mechanics in the driven cavity, Annu. Rev. Fluid Mech. 32 (2000) 93-136.

[26] B.J. Boersma, A 6th order staggered compact finite difference method for the incompressible Navier-Stokes and scalar transport equations, J. Comput. Phys. 230 (2011) 4940-4954.

[27] U. Ghia, K.N. Ghia, C.T. Shin, High-Re solutions for incompressible flow using the Navier-Stokes equations and a multigrid method, J. Comput. Phys. 48 (1982) 387-411.

[28] E.M. Wahba, Steady flow simulations inside a driven cavity up to Reynolds number 35,000, Comput. Fluids 66 (2012) 85-97.

[29] F. Auteri, N. Parolini, L. Quartapelle, Numerical investigation on the stability of singular driven cavity flow, J. Comput. Phys. 183 (2002) 1-25.

[30] C.-H. Bruneau, M. Saad, The 2D lid-driven cavity problem revisited, Comput. Fluids 35 (2006) 326-348.

[31] T.K. Sengupta, A. Sengupta, A new alternating bi-diagonal compact scheme for non-uniform grids, J. Comput. Phys. 310 (2016) 1-25. 